\title{
SUNRISE Pilot Study: \\ Canadian Surveillance Study of Movement Behaviours in the Early Years
}

By

Evan Turner

A thesis submitted to the Faculty of Graduate and Postdoctoral Affairs in partial

fulfilment of the requirements for the degree of

Master of Science

in

Health Sciences

Carleton University

Ottawa, Ontario

(C) 2020

Evan Turner 


\section{Abstract}

There are currently no global estimates of levels of movement behaviours (physical activity, sedentary behaviour, and sleep) in the early years ( $0-4$ years of age). The purpose of this project was to assess the feasibility of the SUNRISE International Surveillance Study of Movement Behaviours in the Early Years by piloting the study in a sample of 4 year-old children in Ottawa. Secondarily, this project aimed to determine the proportion of these participants meeting movement guidelines, compare the proportion of participants meeting screen time recommendations from SUNRISE pilot studies in 8 countries, and contrast movement behaviour data measured by Actical and activPAL accelerometers. Feasibility of recruitment was assessed by the ability to recruit 100 participants attending childcare centres and schools.

Additionally, participants completed study testing measures to assess the feasibility of the SUNRISE study protocols and measurement instruments. Adherence to physical activity recommendations was assessed by accelerometry, and sedentary behaviour/screen time and sleep adherence were assessed with questionnaire data. The overall target sample size was met $(\mathrm{n}=106)$, however the recruitment rate was low at only $16.1 \%$. Issues were also identified with study accelerometers, questionnaires, and cognitive/motor skills tests. Only $19.8 \%$ of participants met the overall movement guidelines. Approximately half of participants from SUNRISE pilot studies were adhering to screen time recommendations and adherence differed by human development index category. Low reliability was found between Actical and activPAL measures of step counts and sedentary/sitting time with no systematic 
biases. The results from this study will contribute to informing the main SUNRISE study. 


\section{Acknowledgements}

I would like to thank all of the study participants and their parents/guardians for their invaluable contribution to this study. As well, thank you to the staff at participating childcare centres and schools for helping us to carry our study and for allowing us into your sites.

I would also like to sincerely thank my supervisor, Dr. Mark Tremblay, for taking me on as a student and for providing me with a wonderful experience over these past two years. Thank you, Mark, for the insights, opportunities, teaching, compassion, and unwavering support throughout my studies.

Thank you to my committee members, Dr. Paul Villeneuve and Dr. Rachel Burns, for your feedback, dedication, and flexibility, which contributed to an amazing graduate experience at Carleton University. Additionally, thank you Dr. Tony Okely and the Early Start group for coordinating the SUNRISE study, for providing the international data used in this thesis, and for your research support.

Thank you to my family and Kaitlyn for your constant encouragement, support, and reassurance during stressful times. Steph, Michelle, Megan, Joel, Sophie, and Katie, this study would not have been possible without your contributions to study coordination, recruitment, data collection, data entry, and analyses. Lastly, thank you to Caro, Kaamel, Ryan, Salomé, Silvia, Taru, and the rest of the HALO Research Group and Department of Health Sciences for your friendship, for sharing your knowledge, and for tolerating my unannounced drop-in conversations. 


\section{Table of Contents}

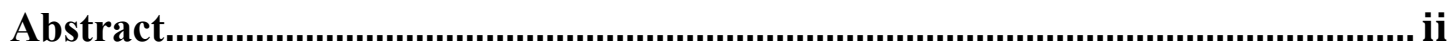

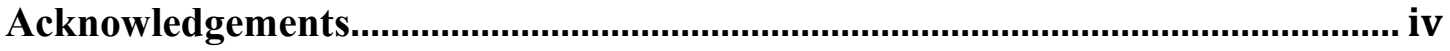

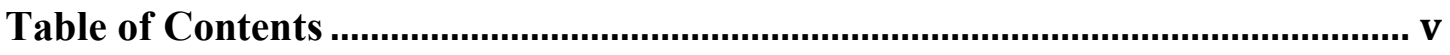

List of Tables..............................................................................................................viii

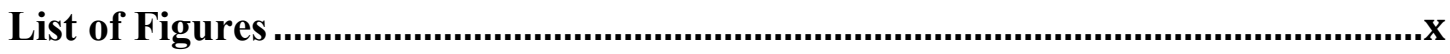

List of Appendices ................................................................................................... $\mathrm{xi}$

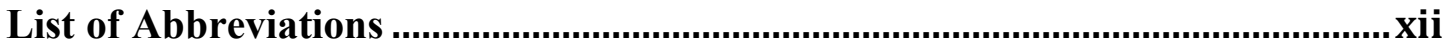

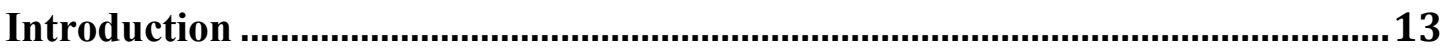

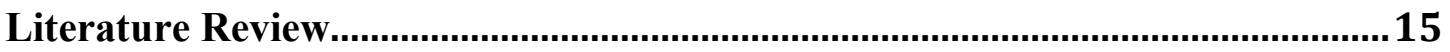

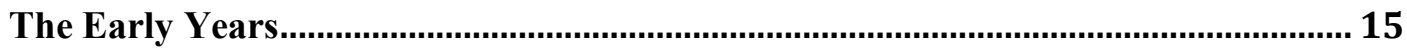

The Developmental Origins of Health and Disease ......................................................... 15

The Capacity-Load Model ............................................................................................................. 17

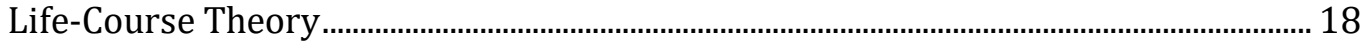

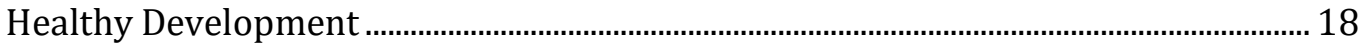

Physical Activity ...................................................................................................... 19

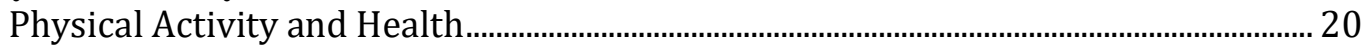

Correlates and Determinants of Physical Activity ............................................................. 22

Measuring Physical Activity …………………………........................................................... 23

Physical Activity Guidelines ............................................................................................... 24

Physical Activity Trends and Prevalence........................................................................ 25

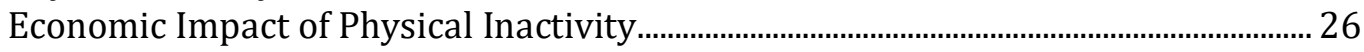

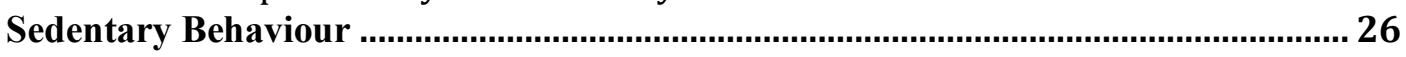

Health Effects of Sedentary Behaviour ……….......................................................... 27

Correlates of Sedentary Behaviour................................................................................. 28

Prevalence and Economic Impact of Sedentary Behaviour ............................................. 28

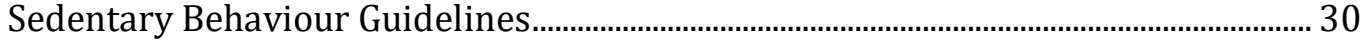

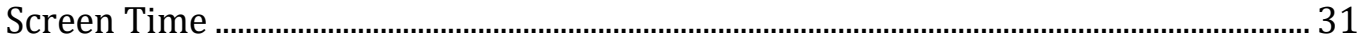

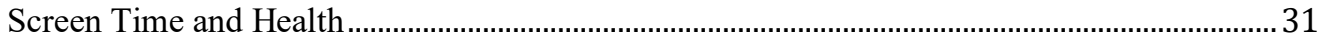

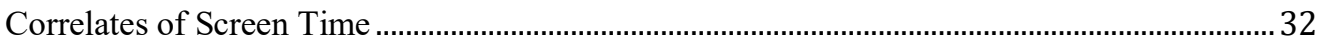

Prevalence of Screen Time................................................................................................................ 33

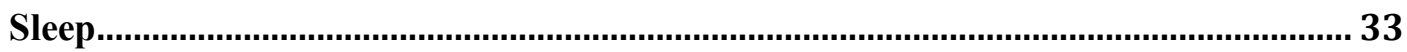

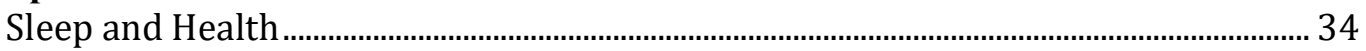

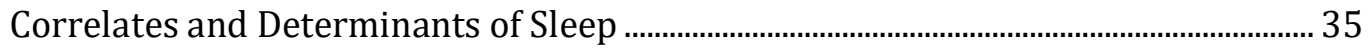

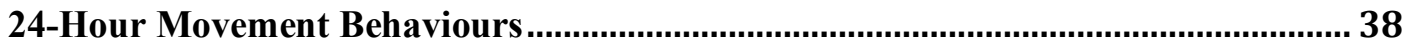

24-Hour Movement Behaviours and Health .......................................................................... 39

24-Hour Movement Guidelines.................................................................................... 41

Prevalence of 24-Hour Movement Guideline Adherence.................................................... 43

Correlates of 24-Hour Movement Guideline Adherence ................................................... 44

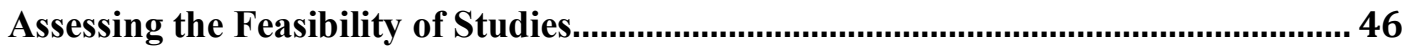

Summary of the Literature and Rationale ................................................................. 47

Research Questions and Hypotheses.................................................................. 49

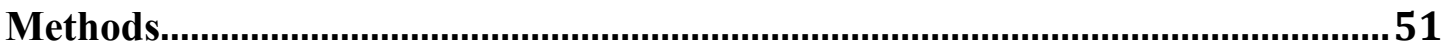




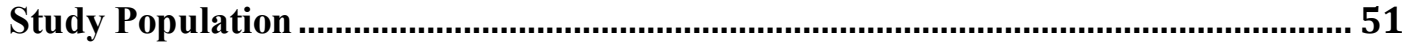

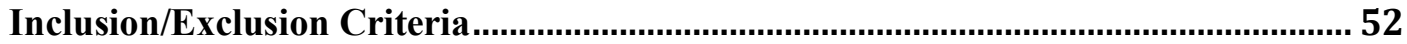

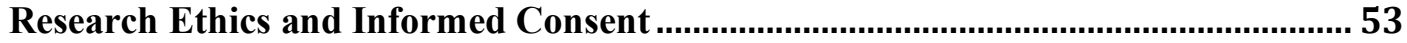

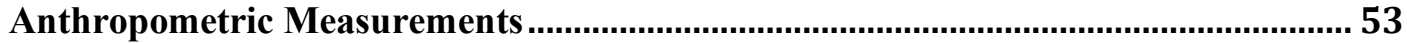

Movement Behaviours - Accelerometer Measurements............................................. 55

Parent/Guardian Questionnaire ............................................................................... 56

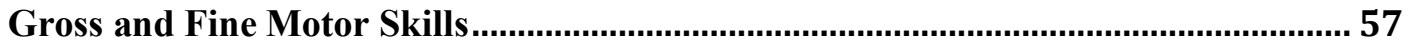

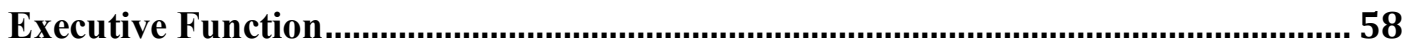

Parent/Guardian and Centre/School Staff Feedback Forms .......................................... 59

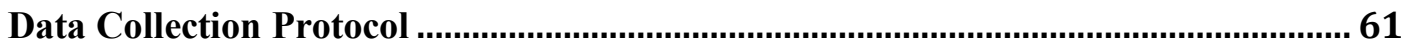

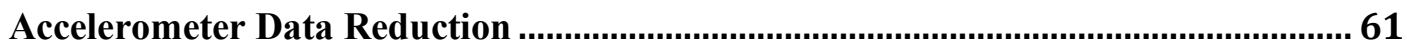

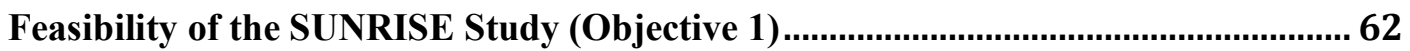

24-Hour Movement Behaviour Adherence (Objective 2) ............................................6 63

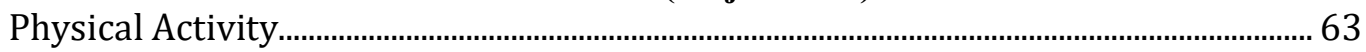

Sedentary Behaviour .......................................................................................................... 64

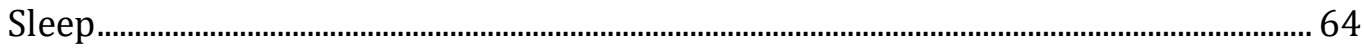

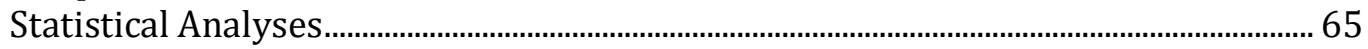

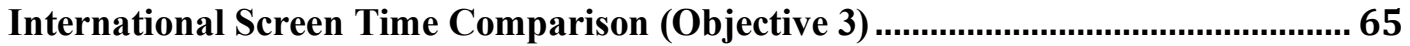

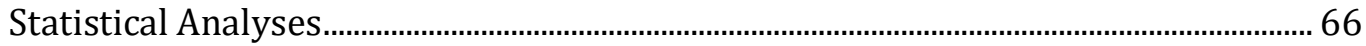

Comparison of Actical and activPAL Physical Activity, Sedentary Behaviour, and

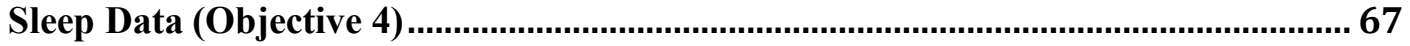

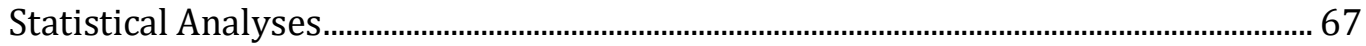

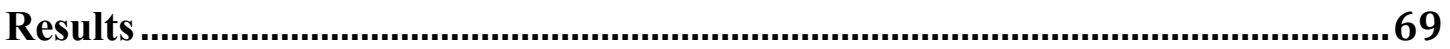

Feasibility of the SUNRISE Study (Objective 1) ......................................................... 70

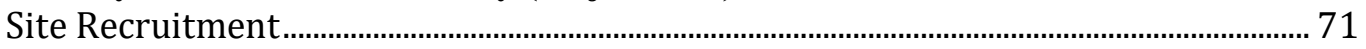

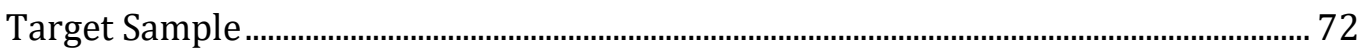

Withdrawals and Exclusions............................................................................................. 72

Feasibility of Accelerometry ......................................................................................... 73

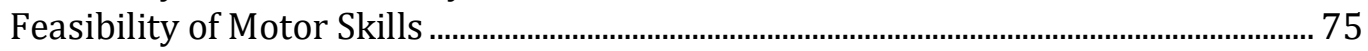

Feasibility of Executive Function ..................................................................................... 75

Feasibility of Parent/Guardian Questionnaire …….......................................................... 76

Staff Feedback Questionnaires...................................................................................... 77

Proportion of Children Adhering to 24-Hour Movement Guidelines (Objective 2) .. 77

Participant Sample for Analyses …………………........................................................... 77

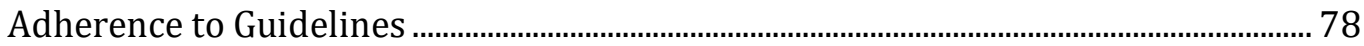

International Screen Time Comparison (Objective 3) .................................................. 80

Actical and activPAL Movement Behaviour Measurement Comparison (Objective 4)

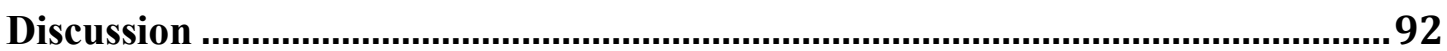

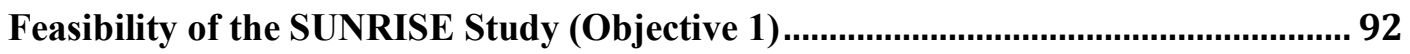

Feasibility of Recruitment ........................................................................................... 92

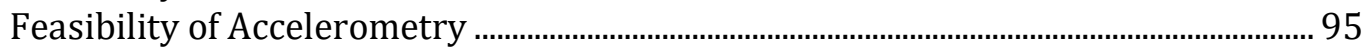

Feasibility of Motor Skills Testing ................................................................................... 97

Feasibility of Executive Function Testing........................................................................... 98

Feasibility of Parent/Guardian Questionnaire ……………………………………......... 98

24-Hour Movement Behaviour Adherence (Objective 2) ..........................................100

International Screen Time Comparison (Objective 3) ................................................104

Comparison of Actical and activPAL Physical Activity, Sedentary Behaviour, and

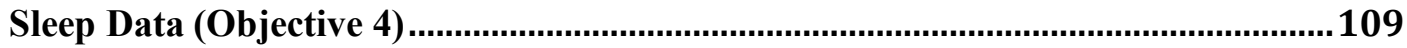




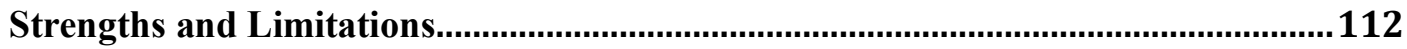

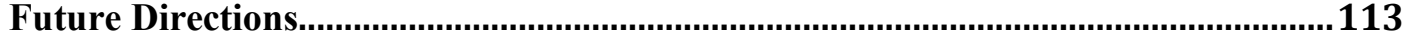

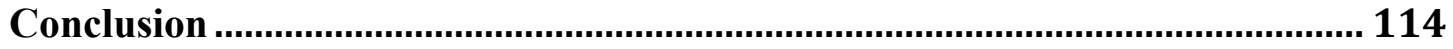

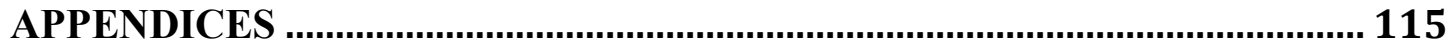

APPENDIX 1. Most-Recent Research Ethics Approvals...........................................115

APPENDIX 2. Parent/Guardian Package Documents ............................................118

APPENDIX 3. Parent/Guardian Questionnaire.....................................................124

APPENDIX 4. 48 Months Ages and Stages Questionnaire Items ..............................129

APPENDIX 5. Parent/Guardian Feedback Form.......................................................130

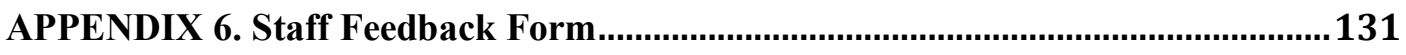

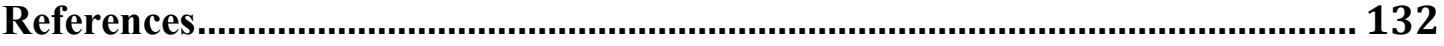




\section{List of Tables}

Table 1. Descriptive characteristics of participants in the Canadian SUNRISE pilot study. .70

Table 2. Descriptive characteristics of participants included in analyses of movement guideline adherence in the Canadian SUNRISE pilot study. .79

Table 3. Daily time Canadian SUNRISE pilot study participants spent in total physical activity, moderate to vigorous intensity physical activity, screen time, and sleep.

Table 4. Descriptive characteristics of participants from the SUNRISE pilot studies of 8 countries included in analyses of screen time adherence.

Table 5. Mean and median daily screen time among SUNRISE pilot study participants from 8 countries.

Table 6. Proportion of participants in 8 SUNRISE pilot studies adhering to screen time recommendations (no more than $1 \mathrm{hr}$ of daily screen time) by country, sex, location, and highest household educational attainment.

Table 7. P-value results of post-hoc pairwise comparison of proportions of participants meeting screen time guidelines ( $\leq 1 \mathrm{hr}$ daily screen time) by SUNRISE pilot study country.

Table 8. Binary logistic regression analysis of screen time recommendation $(<1 \mathrm{hr}$ daily screen time) adherence by Human Development Index, sex, and urban/rural location based on SUNRISE pilot studies in 8 countries (Brazil, Canada, China, Papua New Guinea, South Africa, Sweden, United States, and Zimbabwe; $n=591$ ). 
Table 9. Descriptive characteristics of participants who wore Actical and activPAL accelerometers in the Canadian SUNRISE pilot study........................................ 90 


\section{List of Figures}

Figure 1. Venn Diagram of the proportion (\%) of participants ( $\mathrm{n}=86)$ meeting no recommendations; physical activity, sedentary behaviour, and sleep recommendations; and combinations of recommendations.

Figure 2. Box plots of daily screen time among SUNRISE pilot study participants (n $=591)$ categorized into very high (Canada, Sweden, and United States), high (Brazil, China, and South Africa), and medium to low (Papua New Guinea and Zimbabwe) Human Development Index (HDI) categories.

Figure 3. Bar plot of adherence to screen time recommendations (no more than $1 \mathrm{hr}$ of daily screen time) by very high (Canada, Sweden, and United States), high (Brazil, China, and South Africa), and medium to low (Papua New Guinea and Zimbabwe) Human Development Index (HDI) categories among SUNRISE pilot study participants $(\mathrm{n}=591)$

Figure 4. Bland-Altman plot of differences between daily step counts weighted by wear time measured by activPAL relative to Actical accelerometers $(n=58)$.

Figure 5. Bland-Altman plot of differences between sedentary time/sitting time weighted by wear time measured by activPAL relative to Actical accelerometers $(\mathrm{n}=$ $58)$. 


\section{List of Appendices}

APPENDIX 1. Most-Recent Research Ethics Approvals................................... 115

APPENDIX 2. Parent/Guardian Package Documents....................................... 118

APPENDIX 3. Parent/Guardian Questionnaire............................................ 124

APPENDIX 4. 48 Months Ages and Stages Questionnaire Items ........................ 129

APPENDIX 5. Parent/Guardian Feedback Form ............................................. 130

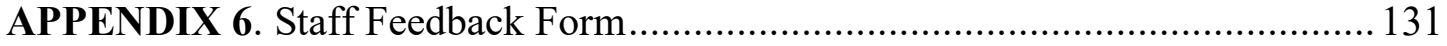




\section{List of Abbreviations}

\begin{tabular}{|l|l|}
\hline Abbreviation & Term \\
\hline ANOVA & Analysis of variance \\
\hline ASQ & Ages and Stages Questionnaire \\
\hline BMI & Body mass index \\
\hline BSID & Bayley Scales of Infant Development \\
\hline CHEO & Children's Hospital of Eastern Ontario \\
\hline CHMS & Canadian Health Measures Survey \\
\hline CI & Confidence interval \\
\hline CSEP & Canadian Society for Exercise Physiology \\
\hline CUREB & Carleton University Research Ethics Board \\
\hline DOHaD & Developmental Origins of Health and Disease \\
\hline EYT & Early Years Toolbox \\
\hline HALO & Healthy Active Living and Obesity research group \\
\hline HDI & Human Development Index \\
\hline ICAD & International Children's Accelerometry Database \\
\hline ICC & Intraclass Correlation Coefficient \\
\hline IQR & Interquartile range \\
\hline ISCOLE & $\begin{array}{l}\text { International Study of Childhood Obesity, Lifestyle and the } \\
\text { Environment }\end{array}$ \\
\hline LPA & Light physical activity \\
\hline MET & Metabolic equivalents of task \\
\hline Mins & Minutes \\
\hline MVPA & Moderate to vigorous physical activity \\
\hline NIH & National Institutes of Health \\
\hline NHANES & National Health and Nutrition Examination Survey \\
\hline OCDSB & Ottawa-Carleton District School Board \\
\hline OCREAC & Ottawa-Carleton Research and Evaluation Advisory Committee \\
\hline OCSB & Ottawa Catholic School Board \\
\hline REB & Research Ethics Board \\
\hline SD & Standard deviation \\
\hline STEPS & STEPwise approach to surveillance \\
\hline SUNRISE & $\begin{array}{l}\text { SUNRISE International Surveillance Study of Movement } \\
\text { Behaviours in the Early Years }\end{array}$ \\
\hline TPA & Total physical activity \\
\hline TV & Television \\
\hline UCDSB & Upper Canada District School Board \\
\hline USA & United States of America \\
\hline WHO & World Health Organization \\
\hline & \\
\hline
\end{tabular}




\section{Introduction}

The early years of life, which many consider to be less than 5 years of age, are a sensitive period of brain and physical development, and arguably, the most critical period for healthy physical, social, motor, and cognitive development. ${ }^{1}$ A child's development in the context of how they move throughout a typical day involves a combination of sleeping, sitting, standing, and different intensities of physical activity. Currently, there is a lack of knowledge about how these 24-hour movement behaviours influence one another, and how they relate to healthy development in young children, especially in low- and middle-income countries.

The World Health Organization (WHO) recently developed movement guidelines for the early years, ${ }^{2}$ based on Canada's ${ }^{3}$ and Australia's ${ }^{4}$ integrated 24Hour Movement Guidelines for the Early Years, to increase awareness of the importance of healthy levels of movement behaviours in young children, and to provide internationally standardized benchmarks. These guidelines will be used by researchers to estimate the prevalence of healthy 24 -hour movement behaviours across different countries and will enable cross-cultural comparisons to further inform local policy and practice. To date, no such studies have been undertaken using these new WHO Guidelines and there is a lack of global estimates of levels of movement behaviours in the early years. ${ }^{2}$

This thesis addresses some of these research gaps, and constitutes the Canadian pilot for the main international surveillance study, the SUNRISE International Surveillance Study of Movement Behaviours in the Early Years (hereafter called SUNRISE). The main SUNRISE study intends to utilize the new 
global movement guidelines to assess the state of healthy movement behaviours among young children across the world and to reveal possible associations with healthy development. This main study will involve the participation of numerous countries with diverse Human Development Index (HDI) backgrounds. HDI is a composite measure of a citizen's capability to live a long and healthy life, to acquire knowledge, and to earn income for a basic standard of living in a given country. ${ }^{5}$ Pilot data aimed at informing the main study were collected from 4 year-old children attending childcare centres in eight countries representing a range of HDI backgrounds (Brazil, Canada, China, Papua New Guinea, South Africa, Sweden, United States, and Zimbabwe). This MSc project was focused on data collection and analysis for the Canadian (very high HDI) arm of the pilot, as well as a comparative analysis of the global screen time pilot data stratified by country HDI. In the Canadian arm of the pilot, data were collected from urban and rural 4 year-oldchildren attending childcare centres and schools in and around the City of Ottawa to assess the feasibility of the SUNRISE study protocol in a Canadian context.

The following section comprises a literature review of the early years, physical activity, sedentary behaviours, sleep behaviours, 24-hour movement behaviours, and assessing the feasibility of studies. The literature review includes research from across the life course (early childhood to adulthood), with emphasis placed on the early years and preschoolers (3-4 years old), and data coming from Canada. 


\section{Literature Review}

\section{The Early Years}

The early years ( $0-5$ years) are a critical period of growth and development that effectively sets the stage for the future acquisition of skills and learning during middle childhood and adolescence and into adulthood. ${ }^{1}$ As a young child develops, they progress through incremental milestones involving the progression of perceptual, motor, cognitive, language, socio-emotional, and self-regulation skills. ${ }^{6}$ Furthermore, as the importance of developmental plasticity during early life is being increasingly recognized, it is clear that the early years are an unparalleled period during which an individual's developmental and health trajectories can be positively or negatively influenced. ${ }^{7}$ Developmental plasticity is defined as the potential for change in intrinsic characteristics during early life in response to external stimuli, ${ }^{8}$ and the first 1000 days post-fertilization are a critical stage when it is possible to exploit this malleability. ${ }^{9}$ Lastly, as will be outlined in the following sections, the early years are a critical period for the promotion of physical activity and reduction of sedentary behaviours. $^{8}$

\section{The Developmental Origins of Health and Disease}

In the mid-1980s, David Barker developed the "Barker Hypothesis", which posited that there was a link between early life environmental factors and diseases developed later in life. ${ }^{10}$ Barker, along with other researchers, noticed that although rising national prosperity was associated with an increased incidence of cardiovascular disease, the poorest groups within developed countries had the highest rates of cardiovascular disease. ${ }^{11-14}$ It was surmised that adverse environmental 
influences in utero and during infancy put individuals at a greater risk of cardiometabolic diseases during adulthood. ${ }^{10}$ The "Barker Hypothesis" was subsequently renamed the Fetal Origin of Adult Disease, and is now known as the Developmental Origins of Health and Disease (DOHaD). ${ }^{15}$ The DOHaD hypothesis states that events during the first 1000 days post-fertilization can positively or negatively influence an offspring's risk of future health issues. ${ }^{9}$ Heindel and Vandenberg compiled a list of common characteristics that environmental influences share. These common characteristics include specific sensitive periods, no apparent defects or malformations at birth, increased risk of disease or dysfunction after a period of latency, increased incidence or earlier onset or increased severity of disease, an effect modulated by genetic background, and potential transmission through the germ-line for intergenerational/transgenerational effects. ${ }^{15}$ In theory, these adverse environmental factors stimulate phenotypic changes in the fetus that allow it to survive, but that also result in long-term health consequences. ${ }^{8}$

By influencing cell differentiation and epigenetic DNA modifications, environmental factors are able to exploit developmental plasticity in utero and throughout the first years of life to influence future health. ${ }^{15}$ Various prenatal maternal factors can influence fetal growth and risk for various non-communicable diseases among their offspring. ${ }^{16}$ These factors include environmental toxin exposure (including air pollution), diet, stress, exercise, age, and longevity. ${ }^{16}$ Moreover, paternal factors can also exert an effect on offspring health during this period. As tissues are formed and stabilize, they become less susceptible to environmental 
influences, and although some tissues are fully developed at birth, others continue to develop well into early childhood (e.g., brain, immune system, metabolic system). ${ }^{15}$

For example, in the early years, research has shown that differing trajectories of body mass index (BMI) growth from birth to 5 years were associated with indices of body composition and markers of cardiometabolic health at 5 years. ${ }^{17}$ Analyses done on the same sample found that weight gain and weight after 48 months were more strongly correlated with adiposity and glucose metabolism at 5 years than the same measures at birth. ${ }^{18}$ Lastly, a longitudinal study following children from birth to 9 years found that children who progressively increased their physical activity levels and maintained low screen time had better health-related quality of life and socioemotional outcomes compared to their peers who maintained low levels of physical activity and maintained low screen time or maintained low physical activity and increased screen time. ${ }^{19}$

\section{The Capacity-Load Model}

The capacity-load model was developed by Jonathan Wells and integrates the life-course etiology to predict future non-communicable disease risk. ${ }^{20}$ The capacityload model introduces the concepts of metabolic capacity and metabolic load. Metabolic capacity refers to physiological traits that are shaped during the first 1000 days by nutrition and growth and, facilitate long-term capacity for metabolic and cardiovascular homeostasis..$^{20}$ Metabolic load refers to nutritional status and lifestyle factors across the life course that challenge homeostasis. ${ }^{20}$ The composite of an increased metabolic load and decreased metabolic capacity acts to increase an individual's risk of non-communicable disease. ${ }^{20}$ In other words, this model provides 
an integration of events occurring in early life and subsequent lifestyle factors in later life to explain susceptibility to non-communicable diseases. ${ }^{20}$ The capacity-load model has been used to explain diabetes prevalence in relation to obesity across cultures. $^{21}$

\section{Life-Course Theory}

The life-course theory also proposes that cross-sectional factors and exposures throughout periods of life accumulate longitudinally in later life. ${ }^{22}$ Consistent with previous theories, the life-course theory emphasizes the importance of exposures in early life and through to adulthood, which influence adult health and mortality risk. ${ }^{8}$ Additionally, the life-course approach recognizes that life experiences are governed by the broader social, economic, and cultural context. ${ }^{8}$ Factors that have been noted to impact health and well-being throughout the life-course include behavioural factors (alcohol consumption, smoking, physical activity, diet and nutrition), environmental factors, and psychosocial factors. ${ }^{8}$

\section{Healthy Development}

Infancy and early childhood are characterized by a number of physical, social, and emotional developmental milestones. ${ }^{8}$ These milestones include the formation of healthy patterns of eating and activity, developing self-regulation/language/cognitive

domains, and developing important learning skills. ${ }^{8}$ Interactions with the environment during the early years shapes the brain, stress systems, capacity to learn, and future health of children. ${ }^{23}$

Research on the state of children's development in Canada suggests that onequarter of children begin school without the necessary physical, social, emotional, 
and cognitive skills to succeed in that context. ${ }^{23}$ For example, approximately $27 \%$ of Canadian senior kindergarteners were found to have at least one measure of either physical, social, emotional, or cognitive development that was considerably below the levels of their Canadian peers. ${ }^{24}$ Of note, potentially two-thirds of these developmental vulnerabilities are preventable ${ }^{25}$ which is critical because persistent vulnerabilities negatively impact academic performance, reduce well-being, and decrease odds of working in a satisfying job as an adult. ${ }^{26}$ Therefore, it is imperative that interventions target the early years to promote healthy development into later childhood and adulthood.

\section{Physical Activity}

Physical activity is defined as any body movement that is produced by the skeletal muscles, and that results in a substantial increase of energy expenditure over resting levels. ${ }^{27}$ Physical activity can come in various forms and in adults often includes walking, sports, active recreation, active work, and domestic tasks. ${ }^{28}$ In contrast, young children often get large amounts of physical activity through more unstructured means such as through play and inventing active ways of passing time. ${ }^{29}$ As children get older they begin engaging in physical activity more through organized sports that can be formal (clubs and teams) or informal (parks and playgrounds). ${ }^{29}$ In addition, physical activity can range from elective activities to activities that are necessary or mandatory within an individual's context. ${ }^{28}$ Physical activity is said to exist along a continuum and can range from light, to moderate, to vigorous, to high intensity. ${ }^{30}$ Physical inactivity is defined as insufficient physical activity levels to meet current physical activity recommendations. ${ }^{31}$ Physical inactivity is thought to be 
responsible for $6-10 \%$ of all deaths from non-communicable diseases ${ }^{32}$, which in 2007 represented an estimated 5.3-5.7 million deaths globally. ${ }^{33}$

\section{Physical Activity and Health}

The health benefits of sufficient physical activity have been noted from as early as 600 B.C. when the Indian physician Susrata prescribed physical activity to prevent and treat diseases and conditions such as obesity and diabetes. ${ }^{34}$ Although Susrata's recommendations were not necessarily based on rigorous scientific evidence, his teachings paved the way for future research that would end up confirming his intuitions.

Results from a systematic review on physical activity and health among adults and older adults provide overwhelming evidence that regular exercise reduces risk for premature all-cause mortality, cardiovascular disease, stroke, hypertension, colon cancer, breast cancer, type 2 diabetes mellitus, and osteoporosis. ${ }^{35}$ These findings suggest that not only is sufficient physical activity an important factor in the prevention of multiple chronic diseases, but due to the commonly observed linear dose-response relationships, increasing levels of physical activity provide increasing health benefits. ${ }^{35}$ In addition, a more recent systematic review of longitudinal studies of physical activity and health found that physical activity had a consistent long-term, positive influence on weight gain, obesity, coronary heart disease, type 2 diabetes mellitus, dementia, and Alzheimer's disease. ${ }^{36}$

Among school-aged children and youth (5-17 years-old), systematic reviews have identified similar health benefits associated with physical activity. ${ }^{37,38}$ There is strong evidence suggesting that in children and youth, total physical activity is 
associated with favourable adiposity, cardiometabolic biomarkers, physical fitness, and bone health. ${ }^{38}$ Weaker evidence also suggests that total physical activity benefits quality of life, motor skill development, and psychological stress. ${ }^{38}$ While generally more and higher intensity physical activity was related to more robust and consistent associations than less and lower intensity physical activity, all patterns of physical activity appeared to provide a health benefit.

Although there has been considerable research evaluating the relationships between physical activity and health among children, youth, and adults, the same cannot be said for research involving the early years. This dearth in research is salient given the importance of the early years as a critical period of rapid physical, cognitive, social, and emotional development. ${ }^{39}$ Despite this, working with the existing minimal low-quality evidence, systematic reviews investigating the relationships between physical activity and health in the early years have still found physical activity to be consistently associated with improved motor development, cognitive development, psychosocial health, cardiometabolic health, fitness, as well as bone and skeletal health. ${ }^{40-44}$ Relationships related to fitness are especially relevant given that it (mainly cardiorespiratory fitness and muscular strength) is an important preventative health factor among children and adolescents. ${ }^{45}$ Additionally, in the review by Carson and colleagues, ${ }^{40}$ it was found that higher intensities of physical activity in preschool-aged children were consistently favourably associated with multiple health indicators (e.g., motor development, psychosocial health, cardiorespiratory fitness, and bone and cardiometabolic health) while lower intensities were not. Although favourable relationships were also observed between 
total physical activity and health indicators, these findings suggest that developmentally appropriate higher intensity physical activity may be necessary for health benefits among young children. ${ }^{40}$ These findings have important potential implications when considering that previous research showed preschool-aged children accumulate most of their physical activity at a light intensity. ${ }^{46-48}$ Consequently, high intensity physical activity is recommended as a target of future research on children in the early years. Moreover, the authors of the systematic reviews heavily caution that the evidence available is of relatively low quality, and there is a need for additional high quality and robust studies. ${ }^{40,44}$

\section{Correlates and Determinants of Physical Activity}

The previously stated systematic reviews also provide evidence that physical activity levels in the early years is predictive of levels in later childhood ${ }^{44}$ and into adulthood. ${ }^{49}$ This tracking emphasizes the need to promote and help establish optimal levels of physical activity in the early years, in order to prevent physical inactivity later in life. In terms of correlates and determinants of physical activity in the early years, a review by Bingham and colleagues ${ }^{50}$ found that greater total physical activity in the early years was positively correlated with male sex, parental physical activity, parental support, and time spent outdoors. The authors also reported that the sex of the child and time spent playing with parents were determinants of total physical activity. Additionally, only sex (male) was found to be a positive correlate of higher intensity physical activity. These findings highlight the influence of various domains of the socio-ecological model and that sex-based disparities, that characterize physical activity in older ages, ${ }^{51}$ may need to be addressed in early childhood. 


\section{Measuring Physical Activity}

A variety of subjective and objective methods are employed to measure physical activity. ${ }^{52}$ Subjective measures (e.g., diaries, recall questionnaires) are indirect methods that are commonly used to assess physical activity due to their versatility, low-cost, and easy use. ${ }^{52}$ However, studies indicate that subjective measures may both over- and under-estimate actual physical activity levels, ${ }^{53,54}$ have poorer reliability and validity, ${ }^{55}$ recall and interpretation bias, ${ }^{52}$ and limitations when recording short, spontaneous bouts of LPA. ${ }^{52}$ Conversely, objective measures (e.g., accelerometers, pedometers, heart rate monitors, doubly labeled water) are used to directly assess specific domains of physical activity and are able to capture a wide range movement data (e.g., steps, mins of activity, intensity of activity, length of bouts of physical activity) ${ }^{55}$ Some research suggests that relative to subjective measures, objective measures are more precise,${ }^{56}$ collect a broader range of data, ${ }^{54}$ and provide a more continuous quantification of free-living physical activity. ${ }^{57}$

Accelerometry is increasingly being used as a method of objectively measuring habitual physical activity. This is especially useful in pediatric populations in which subjective methods tend to overestimate physical activity and may not fully capture the sporadic and intermittent physical activity of a young child. ${ }^{58}$ Accelerometers are able to measure the magnitude and total volume of an individual's movement over a specified period of time, although they are limited to measuring the movement of only the body part that they are affixed to.$^{58}$ Common locations for accelerometer placement include the wrist, ankle, thigh, lower back, hip, and umbilicus. ${ }^{58}$ Among preschoolers, accelerometers have been typically worn over the 
right hip with an elastic belt, ${ }^{59-61}$ however there is a lack of studies adequately investigating the optimal placement of accelerometers in young children. ${ }^{58}$ Inside of the accelerometers, electrical charges generated by accelerations are filtered, converted to activity counts, and then summed over a period, known as an epoch. ${ }^{58}$ The selected epoch length (e.g., $1 \mathrm{sec}, 15 \mathrm{sec}, 60 \mathrm{sec}$ ) is user defined and varies depending on the capability/memory size of the device, desired outcome, and age group of participants. Several studies recommend the use of shorter epochs (i.e., 15 sec or less) for capturing the sporadic bursts of physical activity that are characteristic of preschoolers. ${ }^{62-64}$

\section{Physical Activity Guidelines}

In recognition of the importance of the relationship between physical activity and health, from 1998-2002, Health Canada and the Canadian Society for Exercise Physiology (CSEP) released physical activity guides for school-aged children, youth, adults, and older adults. ${ }^{65}$ In 2011, with leadership from CSEP, new Canadian physical activity guidelines were developed and released across the previously mentioned age groups. ${ }^{66}$ However, it was not until 2012 when guidelines for the early years were developed and released. ${ }^{67}$ These guidelines were developed by an expert panel and informed by a systematic review. ${ }^{44}$ These guidelines included recommendations for infants (aged $<1$ year), toddlers (aged 1-2 years), and preschoolers (3-4 years). Specifically, for preschoolers, these guidelines recommended accumulating at least 180 mins of physical activity at any intensity spread throughout the day and progressing toward 60 mins of energetic play by 5 years of age. These guidelines set the stage for the future development of physical 
activity guidelines in other jurisdictions, guidelines for sedentary behaviour, and 24hour movement guidelines.

\section{Physical Activity Trends and Prevalence}

The implications of the adverse relationships between physical inactivity and health are especially salient given that developed nations have transitioned into societies characterized by high levels of sedentarism, and developing nations are trending along a similar path of decreased physical activity ${ }^{68}$ In what Katzmarzyk and Mason coin as the "physical activity transition", societal changes leading to greater levels of sedentary behaviour conspire to attenuate the life expectancy benefits that countries undergoing economic transition stand to gain. ${ }^{68}$

Recent global data published in 2019 suggests that $77.6 \%$ of boys and $84.7 \%$ of girls aged 11-17 years are insufficiently physically active. ${ }^{69}$ Furthermore, according to 2018 data it is estimated that $23.3 \%$ of adults are physically inactive. ${ }^{70}$

Due to the high prevalence, global reach, and health consequences of physical inactivity, the term "pandemic of physical inactivity" has gained traction. ${ }^{33}$

In terms of the early years, a study by Colley and colleagues estimated that $14 \%$ of 5 year-old Canadian children are meeting the physical activity guideline of 60 mins of daily moderate-to-vigorous physical activity (MVPA) ${ }^{46}$ In addition, a systematic review reported that young children $2-5$ years-old spend 4\%-33\% of their time in light-intensity physical activity and $2 \%-41 \%$ in MVPA. ${ }^{48}$ Despite these varying estimates, there remains a significant lack of data on the early years and there are no current global estimates of physical inactivity prevalence among this age group. 


\section{Economic Impact of Physical Inactivity}

Physical inactivity poses a significant economic burden to healthcare systems. In Canada, the economic burden associated with physical inactivity was estimated at $\$ 6.8$ billion in 2009 , which represented $3.8 \%$ of healthcare spending. ${ }^{71}$ Globally, estimates from 2013 suggest that physical inactivity cost healthcare systems $\$ 53.8$ billion. ${ }^{72}$ In order to address the high prevalence and costs of global physical inactivity, in 2018 WHO member states endorsed a global action plan on physical activity and agreed on the target of a $15 \%$ relative reduction in physical inactivity among adolescents by $2030 .{ }^{69}$

\section{Sedentary Behaviour}

Sedentary behaviour is defined as any waking behaviour characterized by an energy expenditure of less than 1.5 metabolic equivalents of task (METs), while in a sitting, reclining, or lying posture. ${ }^{31}$ A MET is defined as the rate of energy spent, relative to mass, during an activity. ${ }^{73}$ Sedentary behaviour was once considered to be simply the extreme low end of the physical activity continuum. However, it is now being considered a distinct field of research separate from physical activity as shown by emerging evidence highlighting the negative independent health effects of

prolonged sedentary behaviour. ${ }^{74}$ Sedentary behaviour, like physical activity, can come in many diverse forms, such as sitting, watching TV, reading, using computers/cellphones, and travelling in a motorized vehicle. ${ }^{75}$ Additionally, sedentary behaviour is important through its inherent association with physical activity, whereby time spent being sedentary is time lost potentially engaging in physical activity. ${ }^{75}$ This trend is supported by evidence showing inverse associations between 
sedentary behaviour and physical activity. ${ }^{76}$ Screen time is a common source of sedentary behaviour and has been the focus of much of the research surrounding sedentary behaviour. As such, screen time will be described separately in a following section.

\section{Health Effects of Sedentary Behaviour}

Systematic reviews indicate that high sedentary behaviour in adults is associated with depressive symptoms, poorer quality of life, type 2 diabetes, all-cause mortality, and cardiovascular disease mortality. ${ }^{74,77,78}$ A separate systematic review of longitudinal studies found consistent unfavourable relationships between selfreported sedentary behaviour and mortality as well as weight gain from childhood to adulthood. ${ }^{79}$

Among children and adolescents, systematic reviews show that sedentary behaviour adversely impacts obesity, blood pressure, total cholesterol, self-esteem, social behaviour problems, and physical fitness. ${ }^{80}$ In contrast, higher durations of reading and doing homework are positively associated with improved academic achievement. ${ }^{81}$ Another systematic review concluded that there was limited evidence supporting a relationship between volume and patterns of sedentary behaviour and health after accounting for MVPA. ${ }^{82}$ In light of these mixed results, it is important to consider the context of sedentary behaviour as a determinant of associated health outcomes.

With respect to young children, a systematic review found that associations between reading or storytelling and cognitive development were either favourable or null, and that associations between time spent seated/supine and adiposity and motor 
development were primarily unfavorable or null $1{ }^{83}$ The authors of this review speculated that, although the findings suggest that the volume of sedentary time may have a negligible impact on health, the context and mode of sedentary time are important influencers of health effects. Indeed, not all sedentary behaviours are bad and it is therefore important to categorize sedentary behaviours into meaningful categories. Sedentary behaviour in the early years is also concerning because evidence suggests that these behaviours track at moderate levels throughout childhood and into adulthood. ${ }^{84,85}$

\section{Correlates of Sedentary Behaviour}

Research has identified a number of correlates of sedentary behaviour. Among children and adolescents 7-18 years-old, sedentary behaviour has been correlated with age, physical maturity, gender, ethnicity, socioeconomic status, location, week/weekend day, neighborhood satisfaction, access, emotional and physical health status, risk behaviours, family and social influences, physical activity, and nutrition. ${ }^{86}$ In contrast, a systematic review of sedentary behaviour correlates among preschoolaged children was unable to identify consistent correlates of sedentary behaviour. ${ }^{87}$ Recent data from 3-4 year-olds participating in studies included in the International Children's Accelerometry Database (ICAD) suggests that sedentary time is positively correlated with sex (girls), winter, higher parental education, greater daylight hours, and weekdays. ${ }^{88}$

\section{Prevalence and Economic Impact of Sedentary Behaviour}

As a relatively new field, there is somewhat a lack of surveillance data on the prevalence of sedentary behaviour. As previously described, there are numerous 
studies examining the economic costs of physical inactivity; however, there is a dearth of literature on the economic costs of sedentary behaviour due to its conflation with physical inactivity.

Data from the National Health and Nutrition Examination Survey (NHANES) (2001-2016) suggests that between 2007-2016 total sitting time (hrs per day) increased among American adolescents ( $7.0 \mathrm{hrs}$ to $8.2 \mathrm{hrs}$ ) and adults (5.5 hrs to 6.4 hrs). ${ }^{89}$ Based on 2003-2007 data from the Global School-based Student Health Survey, it is estimated that the global prevalence of sedentary behaviour ( 3 or more hrs per day in sitting activities) was $32.7 \%$ for boys and $34.5 \%$ for girls $13-15$ yearsold. ${ }^{90}$ In a systematic review evaluating sedentary time during after-school hours among children and adolescents aged 5-18 years, it was estimated that children spent $41-51 \%$ of their time being sedentary while adolescents spent $57 \% .^{91}$

In the early years, based on the results from a systematic review, it is estimated that young children spend $51.4 \%$ of their waking time in sedentary behaviours with girls being more sedentary than boys. ${ }^{92}$ Data from the Canadian Health Measures Survey (CHMS) suggests that 3-4 year-old children spend 50\% (5.8 hrs) of their waking time per day engaged in sedentary behaviour and 5 year-olds spend $53 \%(6.4 \mathrm{hrs})$ of their waking time being sedentary. ${ }^{46}$ Relative to physical activity, far less research has focused on sedentary time in the early years.

To date, there have not been any studies evaluating the economic cost of sedentary behaviour as an exposure independent of physical inactivity. As previously outlined, evidence suggests that the health effects of sedentary behaviour are independent from those of physical inactivity, therefore it is essential to also treat the 
economic consequences of sedentary behaviour independently. Further research is needed to quantify the costs associated with sedentary behaviour, which are likely to be high given the known prevalence and health effects of the exposure. However, this novel economic analysis is outside of the scope of this thesis.

\section{Sedentary Behaviour Guidelines}

In recognition of the importance of sedentary behaviour, independent of its relation to physical activity, the Healthy Active Living and Obesity (HALO) Research Group at the Children's Hospital of Eastern Ontario Research Institute and the CSEP developed Canadian Sedentary Behaviour Guidelines for Children and Youth. ${ }^{93}$ These guidelines recommend that children and youth (5-17 years-old) should limit recreational screen time to less than $2 \mathrm{hrs}$ per day, with less screen time being better, and to limit sedentary transport, extended sitting time, and time spent indoors throughout the day. ${ }^{93}$ Later, with mounting evidence regarding the importance of sedentary behaviour in the early years, the CSEP released Canadian Sedentary Behaviour Guidelines for the Early Years. ${ }^{94}$ These guidelines stratified recommendations by age groups and recommended that infants ( $<1$ year-old), toddlers (1-2 years-old), and preschoolers (3-4 years-old) should minimize their sedentary time during the day and not be seated or restrained for more than an hour at a time. These guidelines also recommend that children under 2 years-old should have no screen time and those 2-4 years-old should get no more than $1 \mathrm{hr}$ of screen time per day. 


\section{Screen Time}

Screen time is defined as time spent on screen-based behaviours, which can be performed while being sedentary or physically active ${ }^{31}$ and includes TV viewing, playing video games, and using computers/tablets and smartphones. ${ }^{95}$ Screens have now become ubiquitous within our societies, ${ }^{95}$ and in recognition of the significance of screens as direct contributors to sedentary behaviours, screen time is often singled out as the specific sedentary exposure of interest in studies.

\section{Screen Time and Health}

A number of studies and systematic reviews have examined the relationships between screen time and health. In a study of Scottish adults, TV and screen-based entertainment were found to be negatively associated with mental health scores. ${ }^{96} \mathrm{~A}$ longitudinal cohort study found that increased TV viewing in childhood and adolescence was associated with a number of negative health outcomes such as obesity, poor fitness, and raised cholesterol as adults. ${ }^{97}$ With respect to school-aged children, results from a systematic review suggest that higher durations and/or frequencies of screen time/TV viewing/video game use are associated with unfavourable body composition, higher clustered cardiometabolic risk scores, lower fitness, lower self-esteem, and unfavourable behavioural conduct and pro-social behaviour. ${ }^{81}$ Additionally, there was evidence of a dose-response relationship, whereby incrementally less screen time was associated with better health outcomes. ${ }^{81}$ However, findings from these studies were often mixed and included low-quality evidence. 
In the early years, based on the findings of a systematic review examining associations between sedentary behaviour and health indicators, screen-based sedentary behaviours were largely null or adversely associated with adiposity, motor or cognitive development, and psychosocial health. ${ }^{83}$ In addition, a study in 2019 found that screen time in early childhood was associated with poorer social skills. ${ }^{98}$

Despite this evidence suggesting adverse associations between screen time and health, some researchers have concluded that there is weak evidence to support the need for specific screen time limits. ${ }^{99,100}$ These researchers claim that the equivocal evidence for associations between screen time and health among young people calls for an individualized approach where screen time is addressed on a caseby-case basis without overarching recommendations. ${ }^{100}$ They largely attribute the adverse associations of screen time on health among young people to the displacement of time that could be dedicated to other beneficial activities (physical activity, socializing, sleep). ${ }^{100}$ However, the debate surrounding public health approaches to addressing screen time are beyond the scope of this thesis, and discussions regarding screen time will follow Canadian recommendations and guidelines, which endorse a more precautionary and conservative approach to screen time.

\section{Correlates of Screen Time}

A systematic review found non-Caucasian ethnicity to be positively associated with time spent watching TV from preschool to adolescence. ${ }^{101}$ Studies have also shown that sleep and physical activity were inversely related to screen time, whereas energy intake and computer use were positively associated with TV viewing, and age 
was positively associated with TV viewing among preschoolers. ${ }^{87,101}$ Parental rules on screen use in preschoolers were also found to be inversely related with screen time in a small number of studies. ${ }^{87}$ Another systematic review of children under 3 years of age found that child BMI, maternal distress/depression, television viewing time of the mother, and cognitive stimulation in the home were positively associated with screen time. ${ }^{102}$

\section{Prevalence of Screen Time}

Results from the International Study of Childhood Obesity, Lifestyle and the Environment (ISCOLE) indicated that $54.2 \%$ of children in the 12 country study were not meeting screen time guidelines (no more than 2 hrs recreational screen time per day). ${ }^{103}$ Recently, another study revealed that $79 \%$ of 2 year-olds and $95 \%$ of 3 yearolds were not meeting screen time recommendations. ${ }^{104}$ Additionally, data from the CHMS suggest that only $22 \%$ of 3-4 year-old Canadian children are meeting screen time recommendations (no more than $1 \mathrm{hr}$ of screen time per day for 3-4 yearolds). ${ }^{105}$ Another study in Kingston, Ontario, found that less than half (45.8\%) of 2-4

year-old children were meeting screen time-related sedentary behaviour guidelines. ${ }^{106}$ Overall, this high prevalence of excessive screen time potentially exposes a significant proportion of children to a range of associated negative health indicators. Additional research is needed to provide global estimates of screen time in the early years.

Sleep

Sleep, along with physical activity and sedentary behaviour, is an integral component of the movement behaviour continuum. ${ }^{107}$ Sleep medicine began as a field 
largely concerned with sleep disorders. ${ }^{108}$ Recently, sleep deficiency has been receiving increased attention. ${ }^{109}$ Sleep health can be defined in terms of sleep duration, sleep efficiency, timing, alertness/sleepiness, and quality. ${ }^{108}$ Sleep duration refers to the total amount of sleep obtained during a $24 \mathrm{hr}$ period. Sleep efficiency is defined as the ease of falling asleep, staying asleep and returning to sleep. Sleep timing is the placement of sleep within the day. Alertness/sleepiness is the ability to maintain attentive wakefulness. Sleep satisfaction/quality is the subjective assessment of good or poor sleep. ${ }^{108}$ Sleep duration decreases substantially from early childhood to adolescence and shifts timing to occur generally at night. ${ }^{110}$ Sleep time and its relationship with health outcomes has been proposed to be U-shaped whereby both too much and too little sleep can be detrimental to health. ${ }^{111}$ However, the peaks of this curve are not equal, and although the adverse health effects of sleep deficits are well established, excess sleep is more often associated with other health problems. ${ }^{111}$

\section{Sleep and Health}

The majority of systematic reviews evaluating relationships between sleep and health have exclusively examined sleep duration as the sleep characteristic of interest. ${ }^{112}$ However, recently, the other domains of sleep have begun to receive more research interest. ${ }^{112}$ In adults, sleep disruptions have been found to be associated with a variety of negative short- and long-term health outcomes such as increased stress responsivity, psychosocial issues, cardiovascular and metabolic issues, and increased mortality. ${ }^{113}$ Additionally, multiple meta-analyses found that inadequate and excessive sleep were associated with a greater risk for all-cause mortality. ${ }^{114,115}$ Among children and youth, shorter sleep duration was shown to be associated with 
higher adiposity indicators, worse emotional regulation, poorer academic achievement, and lower quality of life/well-being. ${ }^{111}$ A meta-review of sleep and health among children also found substantial evidence supporting the positive relationship between sleep duration and improved adiposity and favourable emotional outcomes. ${ }^{12}$ Weak and inconsistent evidence was found for the relationship between sleep duration and metabolic syndrome in children. ${ }^{12}$

Evidence on early years children indicates that longer sleep duration has favourable associations with adiposity, emotional regulation, growth, screen time, and risks/injuries, but no clear associations with cognitive development, motor development, or physical activity. ${ }^{116}$ This review concluded that there is a significant lack of research on sleep and health in early years children. ${ }^{116}$ In another systematic review, it was found that higher quantity or quality of sleep was associated with improved behavioural/cognitive outcomes in preschoolers although strengths of associations were relatively weak. ${ }^{117}$ Overall, there is a need for more large longitudinal studies to effectively elucidate relationships between various sleep characteristics and health in the early years.

\section{Correlates and Determinants of Sleep}

A systematic review of longitudinal studies revealed that age, screen time, previous sleep patterns, and difficult temperament were determinants of sleep duration in children 4-12 years-old. ${ }^{118}$ This same study found that having a regular weekly schedule was a determinant of sleep timing. ${ }^{118}$ Another cohort study found that black, Hispanic, and Asian children had a greater prevalence of chronic sleep duration curtailment from infancy to mid-childhood. ${ }^{119}$ Children from lower 
socioeconomic status and those from families with increased TV viewing had greater sleep curtailment, defined as shortened sleep duration relative to age-specific means. ${ }^{119}$ Among Canadian preschoolers, based on data from the CHMS, being nonwhite and having a low household income were correlates of short sleep. ${ }^{120}$

Daytime napping is common among young children. As children increase in age, the amount of time allocated to napping decreases. ${ }^{110}$ For example, one study found that $56 \%$ of 3-5 year-old children were found to nap at daycare on 3 consecutive days, and $10 \%$ did not nap on any of the 3 days. ${ }^{121}$ However, by the time a child reaches the age of 6 years, the proportion of children who nap regularly appears to drop to under $10 \%{ }^{122}$

Of concern, it appears that children are getting less sleep over time. Data collected between 1905 to 2008, indicated that sleep duration among children and adolescents is on the decline, with an average decline of -0.75 mins of sleep per year. ${ }^{123}$ This trend puts an increasing number of children and adolescents at increased risk of various adverse health outcomes associated with insufficient sleep. Results from a systematic review indicate that toddlers/preschoolers average $11.9 \mathrm{hrs}$ of sleep per night and children average 9.2 hrs. ${ }^{124}$ Data from the Canadian site of the Health Behaviour in School-aged Children study indicate that $68 \%$ of children $10-13$ yearsold and $72 \%$ of adolescents $14-17$ years-old have sleep durations that meet current recommendations. ${ }^{125}$ In addition, results from the CHMS show that $32.4 \%$ of $6-13$ year-olds and $42 \%$ of $14-17$ year-olds reported trouble going to sleep or staying asleep. ${ }^{126}$ Data from cycles 2 and 3 of the CHMS also suggest that the proportion of Canadians meeting sleep duration recommendations decreases with age, from $81 \%$ in 
preschoolers to $53 \%$ in older adults. ${ }^{120}$ In a recent publication based on CHMS data, it was reported that $83.9 \%$ of preschoolers were meeting sleep duration recommendations. ${ }^{127}$ The literature on sleep in the early years is clearly lacking and there is the need for large international studies to provide meaningful estimates of healthy sleep prevalence in the early years. Furthermore, most research is focused on sleep duration, which downplays the importance of the other characteristics of sleep and potentially overlooks important sleep-health relationships.

\section{Economic Impact of Insufficient Sleep}

Based on an Australian study it was estimated that inadequate sleep cost the country $\$ 45.21$ billion in $2016-2017 .{ }^{128}$ Another study estimates that across the United States, United Kingdom, Japan, Germany, and Canada, insufficient sleep costs nations $\$ 680$ billion every year. ${ }^{129}$ In Canada specifically, it was estimated that insufficient sleep costs Canadians USD \$21.4 billion per year and results in a loss of 80,000 working days each year. ${ }^{129}$

\section{Sleep Guidelines}

In 2015, in recognition of the important health implications of suboptimal sleep, the National Sleep Foundation in the U.S. released sleep duration recommendations for children, adolescents, and adults based on expert concesus. ${ }^{130}$ These recommendations state that for optimal health newborns should get 14-17 hrs of sleep, 12-15 hrs for infants, 11-14 hrs for toddlers, 10-13 hrs for preschoolers, 9-11 hrs for school-aged children, 8-10 hrs for teenagers, 7-9 hrs for young adults and adults, and 7-8 hrs for older adults per day. ${ }^{130}$ The recommendations for newborns to preschoolers include time spent napping. In 2016, the American Academy of Sleep 
Medicine released their own sleep duration recommendations, which generally aligned with those previously published by the National Sleep Foundation. ${ }^{131,132}$ As will be described in the following section, Canadian specific sleep guidelines for children were not released until the release of integrated 24-Hour Movement Guidelines for Children and Youth and 24-Hour Movement Guidelines for the Early Years, which included recommendations for sleep along with physical activity and sedentary behaviour. ${ }^{3,133}$ Before the release of these guidelines some Canadian organizations had issued sleep health recommendations, however they were not developed using robust methods nor primarily evidence informed. ${ }^{134}$

\section{4-Hour Movement Behaviours}

Physical activity, sedentary behaviour, and sleep share numerous common correlates and determinants, ${ }^{133}$ and are the interrelated categories that constitute the movement continuum over a 24 -hour period. ${ }^{107}$ Considering the wealth of evidence indicating that physical activity, sedentary, and sleep behaviours are important for health across all ages, it is clear that movement behaviours across the whole day matter. Not only is it important to maximize physical activity for optimal health, but it is also critical to limit sedentary behaviour and accumulate adequate amounts of quality sleep. In children, on average, total physical activity makes up $\sim 20 \%$ of the day with the remaining proportion consisting of sleep $(\sim 40 \%)$ and sedentary behaviours ( 40\%) ${ }^{135}$ Evidence suggests that the health effects of varying levels of movement behaviours are interactive and unhealthy levels in one domain may attenuate the benefits associated with healthy levels in another domain. ${ }^{135}$ Vice-versa, it is also possible that increasing levels of physical activity can reduce the negative 
effects of inadequate sleep or excessive sedentary behaviour. ${ }^{135}$ In addition, time spent in one movement behaviour may influence time spent in another. For example, data on adolescents from the Youth Risk Behavior Survey System demonstrated that $\geq 60$ mins of daily physical activity and limited computer use (sedentary behaviour) were associated with higher odds of getting sufficient sleep. ${ }^{136}$

\section{4-Hour Movement Behaviours and Health}

Further supporting the importance of all movement behaviours, a crosssectional study of American children from the Midwest found that the odds of being obese decreased with the increasing number of movement behaviour recommendations met. ${ }^{137}$ Given that movement behaviours exist as domains on the same finite scale (24-hour period); physical activity, sedentary behaviour, and sleep are inherently collinear and codependent. ${ }^{138}$ Therefore, correlational studies that include multiple movement behaviours in regression models are prone to spurious and inconsistent results. ${ }^{139}$ To address this limitation, compositional analyses were introduced to the field of movement behaviours, whereby the effects of a behaviour are interpreted as a proportion relative to the other behaviours that compose the finite whole. ${ }^{138}$ This is in contrast to traditional analyses where behaviours are assumed to be independent of one another. Based on compositional analyses of physical activity, sedentary time, and sleep duration data of children and youth from the CHMS cycles 1-3, it was found that the composition of movement behaviours was associated with a variety of health indicators such as obesity risk markers, behavioural strengths and difficulties scores, systolic blood pressure, aerobic fitness, and multiple cardiometabolic risk biomarkers. ${ }^{139}$ Additionally, it was found that replacing MVPA 
with increased time spent in other movement behaviours had the greatest negative impact on health. ${ }^{139}$

Another compositional analysis using NHANES data found that the distribution of time spent in sleep, sedentary behaviour, light physical activity (LPA), and MVPA was significantly associated with BMI, waist circumference, triglycerides, plasma glucose, plasma insulin, and systolic and diastolic blood pressure in adults. ${ }^{138}$ These analyses found that the strongest adverse effects were evident when sedentary behaviour replaced MVPA. ${ }^{138}$ Overall, the authors found that decreasing sedentary time and maintaining or increasing MVPA were associated with favourable cardiometabolic profiles and health risks were greater when time spent in MVPA was exchanged for time in sedentary behaviour compared to when it was exchanged for LPA. ${ }^{138}$ An additional, compositional analysis that used CHMS data from adults found beneficial associations between increased time spent in MVPA relative to time spent in other behaviours for BMI, waist circumference, aerobic fitness, resting heart rate, high-density lipoprotein cholesterol, triglycerides, blood glucose, and insulin levels. ${ }^{140}$ Additionally, a systematic review of isotemporal substitution studies found that exchanging sedentary time for time spent in LPA or MVPA was associated with reduced mortality risk, with greater benefits associated with time spent in higher intensities of physical activity. ${ }^{141}$ Following a similar pattern, favourable adiposity indicators were associated with sedentary time being substituted for time spent in physical activity. ${ }^{141}$

A systematic review of combinations of movement behaviours and health outcomes found that children and youth with high physical activity, high sleep, and 
low sedentary behaviour had more favourable adiposity and cardiometabolic risk profiles than those with low physical activity, low sleep, and high sedentary behaviour. ${ }^{142}$ Favourable combinations of two movement behaviours (high physical activity/high sleep and high physical activity/low sedentary behaviour) were associated with benefits to cardiometabolic health, adiposity, and fitness compared to corresponding unfavourable movement behaviour combinations. ${ }^{142}$ High sleep and low sedentary behaviour was associated with improved cardiometabolic health relative to low sleep and high sedentary behaviour. ${ }^{142}$ Furthermore, similar to other studies, health benefits were found to be most consistently associated with physical activity, and especially MVPA. ${ }^{142}$

In the early years, a systematic review found that ideal combinations of movement behaviours are associated with a range of health and developmental outcomes. ${ }^{107}$ Among preschool-aged children, it was found that low sedentary behaviour and high physical activity were associated with favourable motor development and fitness, and both favourably and not associated with adiposity. ${ }^{107}$ Additionally, high sleep and low sedentary behaviour were favourably associated with adiposity among infants and toddlers. ${ }^{107}$ Notably, the systematic review had very few high quality studies to draw data from and concluded additional studies are required to reveal the complete spectrum of health outcomes related to combinations of movement behaviours across the early years.

\section{4-Hour Movement Guidelines}

In light of the substantial evidence supporting the 24-hour movement paradigm, in 2016 Canada released 24-Hour Movement Guidelines for Children and 
Youth (5-17 years). ${ }^{133}$ These guidelines included recommendations for healthy levels of physical activity, sedentary behaviour, and sleep and generally followed already established guidelines for each individual behaviour. Following the release of these guidelines, Canada, ${ }^{3}$ Australia, ${ }^{4}$ and New Zealand ${ }^{143}$ released 24-hour movement guidelines for the early years (0-4/5 years) in 2017. Since then, South Africa, ${ }^{144}$ the United Kingdom, ${ }^{145}$ and the United States ${ }^{146}$ have also released their own movement guidelines for the early years, although the guidelines from the United Kingdom focused solely on physical activity on the decision of Chief Medical Officers despite the evidence presented for the 24-hour paradigm outlined by researchers.

Subsequently, the WHO released their own movement behaviour guidelines for the early years in 2019, modelled after the Canadian Guidelines. ${ }^{2}$ These guidelines provide benchmarks that can be applied in cross-cultural population surveillance studies of movement behaviours, to monitor trends and inequalities over time, and provide evidence that will inform interventions targeting movement domains where children are failing. ${ }^{147}$ Moreover, these guidelines address one of the proposed actions for the WHO Secretariat in the WHO's Global Action Plan on Physical Activity 2018-2030, which outlines the need to "Partner and support the development of new guidance and protocols on the monitoring of physical activity and sedentary behaviours in children aged $<5$ years" ${ }^{28}$ These WHO guidelines include separate recommendations for infants (less than 1 year), children 1-2 years of age, and children 3-4 years of age. ${ }^{2}$ Specifically, with regard to children 3-4 years of age, the guidelines recommend that children engage in at least 180 mins of physical activity with at least 60 mins being MVPA; not be restrained for more than $1 \mathrm{hr}$ at a time and 
engage in no more than $1 \mathrm{hr}$ of sedentary screen time; and have 10-13 hrs of good quality sleep with regular sleep and wake-up times. ${ }^{2}$ These guidelines are essentially identical to the previously published Canadian early years guidelines as well as those published by other countries. These global guidelines provide movement behaviour benchmarks that are applicable across countries throughout the world and provide a usable framework for surveillance of movement behaviours in the early years.

\section{Prevalence of 24-Hour Movement Guideline Adherence}

Given the publication of various 24-hour movement guidelines, it is possible for researchers to assess the adherence of individuals to physical activity, sedentary behaviour, and sleep recommendations. Results from the ISCOLE indicate that among 9-11 year-olds, the global prevalence of children meeting all movement recommendations was $7 \%$ while $19 \%$ of children were meeting none of the recommendations. ${ }^{148}$ In this study, Brazil, Portugal, and the USA (all 29\%) had the highest proportion of children meeting no recommendations. ${ }^{148}$ Based on CHMS data, only 3\% of Canadian 10-17 year-olds were meeting all three movement recommendations, $25 \%$ were meeting two recommendations, $51 \%$ were meeting one recommendation only, and $21 \%$ were meeting no recommendations. ${ }^{149}$ Sleep was the most frequently met recommendation (66\%), with MVPA (35\%) and screen time (8\%) adherence much lower. ${ }^{149}$ Also from CHMS data, the proportion of 5-11 yearolds meeting $3,2,1$, and no recommendations was $37 \%, 46 \%, 20 \%$, and $4 \%$, respectively. ${ }^{150}$

Among preschoolers, Australian data indicate that $14.9 \%$ of children are meeting all 3 recommendations. ${ }^{151}$ Ninety three percent were meeting physical 
activity recommendations, $88.7 \%$ were meeting sleep recommendations, and $17.3 \%$ were meeting screen time recommendations. ${ }^{151}$ From CHMS data, it was found that among preschool-aged Canadians (3-4 years), only 12.7\% were meeting all 3 movement guidelines for the early years and $3.3 \%$ were meeting none of the recommendations. ${ }^{127}$ In this study, sleep duration (10-13 hrs of daily sleep) was the most commonly met recommendation ( $83.9 \%)$, followed by physical activity (180 mins of total physical activity and 60 mins of daily MVPA; $61.8 \%$ ) and screen time (no more than $1 \mathrm{hr}$ of daily screen time) was the least frequently met recommendation (24.4\%). ${ }^{127}$ In another study by Carson and colleagues, a similarly low (5\%) proportion of 3 year-olds from Edmonton were meeting overall guidelines, however only $19.3 \%$ were meeting physical activity recommendations and slightly over half were meeting screen time recommendations. ${ }^{152}$ Among toddlers (1-2 years-old), a study based in Edmonton found that the majority of toddlers were meeting physical activity (99.3\%) and sleep (82.1\%) recommendations, while only $15.2 \%$ met screen time recommendations. ${ }^{153}$ Only $11.9 \%$ met the overall movement guidelines. ${ }^{153}$ Of note, there have yet to be any international studies of movement behaviours in the early years, therefore it is not yet possible to provide global estimates of the prevalence of adherence to movement behaviours in the early years.

\section{Correlates of 24-Hour Movement Guideline Adherence}

Although the correlates and determinants of individual movement behaviours have been relatively well studied, there is a dearth of evidence related to the correlates and determinants of having healthy levels of movement behaviours in combination. One study of children (9-11 years-old) in Mozambique found rural location to be 
associated with longer sleep duration and shorter recreational screen time relative to those from urban locations. ${ }^{154}$ Additionally, parental education, school location, and outdoor time were found to be associated with meeting all 3 movement guidelines. ${ }^{154}$ In a study of South Koreans 12 years and older, findings showed that older age, female sex, and living in metro Seoul were associated with unfavourable combinations of movement behaviours across all age groups. ${ }^{155}$ Data from the UK suggest that adolescent girls from the highest income tertile were more likely to meet all 3 movement recommendations relative to girls in the lowest tertile. ${ }^{156}$ Adolescent girls with depressive symptoms were less likely to meet all 3 recommendations than their peers without any depressive symptoms, and adolescent boys with obesity and those with depressive symptoms were less likely to meet all 3 recommendations relative to their normal weight and no depressive symptom peers. ${ }^{156}$

In the early years, a follow-up study of 5 year-olds from Singapore found that higher maternal physical activity and lower maternal TV viewing were associated with their children adhering to all movement guidelines. ${ }^{157}$ In a study of American 34 year-olds, it was found that those with African American ethnicity or living at or below the poverty line were less likely to meet sleep, sedentary time, or all 3 movement guidelines relative to their peers. ${ }^{158}$ It is important to recognize that diversity in results across studies may have been driven by differences in study design and sample sizes. Furthermore, correlates are context-dependent, which limits generalizing associations outside of the population being studied. To date, there have not been any Canadian studies specifically examining potential correlates of 24-hour movement behaviours in the early years. 


\section{Assessing the Feasibility of Studies}

Feasibility is a broad concept that in the context of this thesis concerns refining aspects of a study's design and protocol to inform the planning of a future main study. ${ }^{159}$ Some important functions of feasibility studies are to assess whether acceptable recruitment/retention rates can be achieved and to assess the acceptability of specific interventions or measures. ${ }^{160}$ Orsmond and Cohn developed a list of main objectives that feasibility studies will generally include as points of focus. ${ }^{161}$ These objectives include the evaluation of recruitment ability and associated sample characteristics, the optimization of data collection protocols and study outcome measures, the evaluation of the acceptability of study interventions and procedures, the evaluation of the ability to implement the study and intervention, and the preliminary evaluation of study results.

Potential outcomes of interest in feasibility studies are quite diverse and can include satisfaction, perceived demand, factors affecting ease of intervention implementation, positive/negative effects on participants, and ability of participants to carry out study activities. ${ }^{162}$ For example, in a cross-sectional study assessing the feasibility of a study objectively measuring physical activity and sedentary behaviour among 2-3 year-olds and their parents, the authors reported results on recruitment rates and compliance with accelerometer wear protocols. ${ }^{163}$ In another study, the feasibility of physical activity assessment with a wearable device among 4-10 yearolds was assessed with data from questionnaires asking about the child's experience and attitudes regarding the device. ${ }^{164}$ Importantly, carrying out feasibility studies can help to prevent wasting funding and time in main studies that fail to be completed by 
providing the opportunity to identify and address issues related to research questions or study design beforehand. ${ }^{160}$

\section{Summary of the Literature and Rationale}

In summary, the early years are a critical period of development in which different exposures can predispose children to future health outcomes. Although the literature base for the implications of physical activity, sedentary behaviour, and sleep are relatively well established for adults and children and youth, these movement behaviours have been far less studied in the early years. Despite this lack of research, the limited literature suggests that unfavourable levels of movement behaviours are associated with several negative health outcomes in the early years. ${ }^{107}$ These movement behaviours have also been found to track from early childhood to later in life, which has implications given what we know about the health associations of movement behaviours in older age groups. A Canadian nationally representative study that assessed movement behaviours in the early years has been carried out, ${ }^{127}$ however, there remains a dearth of global data and there have yet to be any largescale international surveillance studies. Therefore, we still do not know whether young children across the world have healthy levels of movement behaviours, or whether they have unfavourable levels that put them at risk for negative health outcomes. To aid the collection of international data on movement behaviours in the early years, the WHO has recently released Guidelines on Physical Activity, Sedentary Behaviour and Sleep for Children Under 5 Years of Age. ${ }^{2}$ These new guidelines provide international benchmarks that countries across the world can apply 
to assess the prevalence of young children meeting healthy movement behaviour recommendations in their regions.

Given the previously highlighted paucity of global data on levels of movement behaviours in the early years, the SUNRISE study was designed to address this research gap. The SUNRISE study aims to collect global data on movement behaviours in the early years by organizing standardized surveillance studies of movement behaviours in a broad range of countries across the world. Canada is participating in the SUNRISE study to collect new data using this standardized protocol in order to contribute to global estimates and comparisons of movement behaviours based on the harmonized data. Additionally, Canada is piloting the SUNRISE study protocol before completing the main study to identify specific aspects of the standardized protocol that may be challenging in the Canadian context. Several other countries are also conducting pilot testing to further inform the development of the standardized study protocol that will be applied in the main surveillance study. 


\section{Research Questions and Hypotheses}

The SUNRISE study aims to collect global data on movement behaviours in the early years by organizing standardized surveillance studies of movement behaviours in a broad range of countries across the world. The SUNRISE study protocol is currently being piloted in several countries. This MSc project will examine pilot data from the SUNRISE study to address the following specific questions:

1) Are the SUNRISE study measurement instruments and protocols feasible in a very high Human Development Index (HDI) context (Canada) using a convenience sampling approach?

Hypothesis: The study measures and protocols were hypothesized to be feasible in our very high HDI context. Precedence set by the success of the CHMS $^{105}$ suggests that large-scale collection of accelerometer and questionnaire based data on physical activity, sedentary behaviour, and sleep in young children is feasible in a Canadian context.

2) What proportion of the SUNRISE pilot sample of Canadian 4 year-old children meet the WHO Guidelines on Physical Activity, Sedentary Behaviour and Sleep for Children Under 5 Years of Age? ${ }^{2}$

Hypothesis: It is likely that a low proportion of Canadian young children are meeting the new 24-Hour Movement Guidelines. ${ }^{2}$ Results from recent studies on the Canadian 24-Hour Movement Guidelines for the Early Years ${ }^{127,153}$ revealed that a low proportion of children were meeting all three recommendations for healthy movement behaviours. A high proportion of 
children were meeting physical activity and sleep recommendations, but a low proportion were meeting recommendations for screen time (sedentary behaviour). As the new WHO guidelines have been modeled after Canada and Australia's movement guidelines, we expected to see similar results.

\section{3) Does the proportion of 4 year-old children meeting screen time} recommendations differ by sex, HDI, or urban/rural location based on the pilot study results of eight countries?

Hypothesis: Based on the results from several studies on the Canadian 24Hour Movement Guidelines for the Early Years that found no sex differences in levels of healthy movement behaviours, ${ }^{127,153}$ it was hypothesized that there would not be any sex differences in the proportion of young children meeting the new guidelines in the current study. In addition, based on the findings from the Global Matrix 2.0, ${ }^{165}$ which assigned higher grades for childhood physical activity and sedentary behaviour to medium to low HDI countries, it was expected that medium to low HDI and rural status would be related to higher proportions of children meeting screen time recommendations.

4) Do the activPAL and Actical accelerometers produce similar physical activity, sedentary behaviour, and sleep results when worn concurrently in young Canadian children?

Hypothesis: It was hypothesized that the devices would produce similar movement behaviour results since both devices have been validated for their accuracy of recording movement behaviour data in young children. ${ }^{166,167}$ 


\section{Methods}

The SUNRISE study is an international surveillance study organized by the Early Start group at the University of Wollongong, Australia. To inform the main surveillance study, pilot studies were carried out in several countries with a range of HDI backgrounds (Brazil, Canada, China, Papua New Guinea, South Africa, Sweden, United States, and Zimbabwe). Following a standardized study protocol, anthropometric, movement behaviour, executive function, motor skills, and demographic data were collected from 4 year-olds in the pilot studies. Informed consent was provided by the children's parents/guardians. Pilot studies had a target sample size of 100 participants, with an equal number of boys and girls coming from urban (50) and rural (50) locations as determined by home address. The study methods and measures were decided a-priori by a group of physical activity experts on the leadership committee of the SUNRISE study. As a sub-objective of the SUNRISE study, data related to executive function and motor skills were collected to investigate possible associations between adherence to movement guidelines and cognitive and motor development. However, analyses of these data are outside of the scope of this thesis, so results from the executive function and motor skills testing are only presented in terms of feasibility. The Canadian SUNRISE pilot study was based out of the Children's Hospital of Eastern Ontario Research Institute.

\section{Study Population}

A convenience sampling approach was used to recruit 4 year-old children attending childcare centres and schools in and around the City of Ottawa. Participants for whom parent/guardian informed consent was obtained were assigned a de- 
identified unique participant ID. Participating childcare centres included publicly and privately funded establishments, and participating schools were from the OttawaCarleton District School Board (OCDSB) and the Ottawa Catholic School Board (OCSB). The OCDSB and OCSB are the two main public-school boards that service the City of Ottawa. Childcare centres and schools were from urban and rural locations. The second character of the child's home postal code was used to classify them based on urban or rural residency, with the second character being a ' 0 ' used to denote a rural address. Rural classifications were then cross referenced with 2016 census data on population density for each postal code,${ }^{168}$ with rural areas defined as having a population $<1000$ people and a density of $<400$ people per square kilometer. ${ }^{169}$

\section{Inclusion/Exclusion Criteria}

The parents/guardians of all 4 year-old children attending participating childcare centres and schools were invited to participate in the study. Apart from the age criteria, children were also excluded if they had a physical disability that precluded them from wearing hip-worn accelerometers, or if they had a developmental disorder that precluded them from understanding directives on how to complete executive function and motor skills testing. Additionally, all study documents were in English only, so non-English speaking children were likely excluded non-intentionally. Children who dissented to participation in study measures were withdrawn from the study. 


\section{Research Ethics and Informed Consent}

Research ethics approval was obtained from the Children's Hospital of Eastern Ontario Research Ethics Board (CHEO REB; 18/19X), the Carleton University Research Ethics Board (CUREB; \#109584), and the Ottawa-Carleton Research and Evaluation Advisory Committee (OCREAC). The most recent copies of research ethics approval certificates/notices are provided in APPENDIX 1.

Childcare centres and schools were contacted by phone, email, and/or in person to inquire about their willingness to participate in the study. Locations agreeing to participate were then provided parent/guardian study packages for all 4 year-old children attending their institution. These packages included: a cover letter, study information sheet, contact information sheet, and a parental consent form (APPENDIX 2). Staff at childcare centres and schools were responsible for distributing packages to parents/guardians of 4 year-old children under their care/in their classes. Parents/guardians who consented to their child's participation in the study were instructed to complete the contact information sheet and consent form and to return the documents to the staff via their children.

\section{Anthropometric Measurements}

The assessment of growth (e.g., height, weight) among children is a critical component of any clinical evaluation and is important for the evaluation of overall health, development, and nutritional status. ${ }^{170}$ To assess growth, recorded anthropometric values are typically compared against a certain reference population with specific cut-points to define over-nutrition and under-nutrition. ${ }^{170}$ Between 1997-2003 the WHO Multicentre Growth Reference Study collected growth and 
related data from 8,500 children from a broad range of countries (Brazil, Ghana, India, Norway, Oman, and the USA), that provided the basis of international growth curves that are now commonly used. ${ }^{171}$

The height of participants was measured with a Seca 213 stadiometer (Seca North America, Chino, CA) and weight was measured with a Conair Thinner TH178WC digital scale (Conair Consumer Products, Woodbridge, ON). Participants were asked to remove their shoes and excessively bulky clothing during the anthropometric measurements. Height was measured to the nearest $0.1 \mathrm{~cm}$ with the child standing upright with their head in the Frankfort plane. Weight was measured to the nearest $0.1 \mathrm{~kg}$ with the child standing squarely on the scale and not holding onto any supports. Two measures of height and weight were taken. If the two measures of height differed by more than $0.5 \mathrm{~cm}$, or the two measures of weight differed by more than $0.25 \mathrm{~kg}$, then a third measure was taken. An average of the two closest measures for both height and weight were calculated and used in the analyses. Based on the participant's age (in days) at the time of the anthropometric measurements, average height value, average weight value, sex and age specific BMI z-scores were calculated using the WHO "anthro" package (V0.9.1; Schumacher et al.) for R. The "anthro" package utilizes the WHO Multicentre Growth Reference Study data as the reference population for calculating anthropometric z-scores. BMI z-scores were then used to categorize participants as wasted, normal-weight, at-risk of overweight, overweight, or obese (wasted z-score $<-2.0$; normal-weight -score $\geq-2.0$ and $\leq 1.0$; at-risk of overweight $>1.0$ and $\leq 2.0$; overweight $>2.0$ and $\leq 3.0$; obese $>3.0$ ). ${ }^{172}$ 


\section{Movement Behaviours - Accelerometer Measurements}

In this study the physical activity level of participants was assessed using Actical (Philips Respironics, Bend, OR, USA) and activPAL (PAL Technologies, Glasgow, Scotland) accelerometers worn continuously for 3 weekdays. Both devices were initialized to begin recording at 12:00 am on the morning of the first day of data collection. Both devices were set to record activity data in 15 second epochs. Acticals were explained to be worn over the right hip with a nylon belt and were to be removed only when engaging in water-based activities (e.g., swimming, bathing, showering). activPALs were worn on the right anterior mid-thigh and were affixed with two sheets of Tegaderm (3M Canada, London, ON, Canada). activPALs were waterproofed with a nitrile sleeve and wrapped in Tegaderm prior to being affixed to the participant, which allowed them to be worn continuously even during water activities.

Actical accelerometers are omnidirectional piezoelectric accelerometers. The piezoelectric sensor in the Actical detects accelerations through a ceramic case that transfers vibrations to the sensor, which results in the sensor releasing a charge proportional to the acceleration force. ${ }^{173}$ This charge is filtered and converted to activity counts, that are then summed over the user-defined epoch period. The output of Acticals includes activity counts and step counts. ${ }^{173,174}$ Actical accelerometers can be worn on the ankle, wrist, or hip, although the sensor in the device is most sensitive to acceleration in the vertical plane when worn on the hip. ${ }^{173}$ Validation studies of the Actical in preschoolers, which used a hip placement and $15 \mathrm{sec}$ epochs, found the

devices to be a valid tool for measuring physical activity in young children. ${ }^{167,175}$ 
activPAL devices are relatively new tri-axial accelerometers and inclinometers that are used to measure free-living physical activity and sedentary behaviour. ${ }^{176,177}$ activPALs are designed to be worn on the thigh and uses its internal accelerometer to sense limb position and activity. ${ }^{176}$ The device is then able to use the incline of the thigh to classify activity into postural categories that include sitting/lying, standing, and stepping. ${ }^{178}$ The activity output from activPALs include cadence, acceleration counts, step counts, and METs (calculated from cadence). ${ }^{179}$ An advantage of the activPAL device is that it can discriminate between standing and sitting/lying down sedentary behaviour, whereas other accelerometers are unable to do so. ${ }^{176}$ Studies have validated the activPAL for assessing physical activity and sedentary behaviour in children ${ }^{166}$ and adults, ${ }^{180,181}$ however there is a lack of clarity regarding the accuracy of the devices in determining different intensities of physical activity among preschool-aged children. ${ }^{176,179,182}$ There are currently no validated activPAL physical activity intensity cut-points for preschool-aged children.

\section{Parent/Guardian Questionnaire}

A modified WHO STEPwise approach to surveillance (STEPS) ${ }^{183}$ parent/guardian questionnaire was sent home with participating children for their parents/guardians to complete. The first section of the questionnaire contained items related to the name of the child's childcare centre/school and the parent/guardian's relationship (i.e., mother, father, grandmother, grandfather, legal guardian) to the child. The second section included items regarding the sex and age of the child and parent/guardian, highest level of household educational attainment, and ethnic background of the child. The third section asked a range of questions related to the 
child's physical activity, screen time, sedentary behaviour, and sleep. In addition, this section asked questions about the parent/guardian's use of electronic screen devices around the child. The final section included questions pertaining to their child's sedentary travel time and time spent outside. The questionnaire was designed to be completed by the parent/guardian in roughly 15-20 mins. A complete copy of the parent/guardian questionnaire is in APPENDIX 3.

\section{Gross and Fine Motor Skills}

The Ages and Stages Questionnaire (ASQ; Paul H. Brookes Publishing Co., Baltimore, MD) is a validated ${ }^{184-186}$ screening tool used to assess the development of young children. ${ }^{187}$ The ASQ consists of 21 age-specific questions that cover ages 166 months and includes domains such as communication, gross motor, fine motor, problem solving, and personal social skills. ${ }^{187}$ Domains are assessed with 6 questions that cover important milestones and that are to be answered with either "Yes", "Sometimes", or "No". ${ }^{187}$ The ASQ is generally completed by parents in the home setting.

In this study, the gross and fine motor sections of the ASQ-3 for 48 months were used to assess the gross and fine motor skills of participants. At childcare centre/school visits, participating children were asked to complete each of the gross and fine motor skills items (except for ladder climb and slide), and items were scored by trained research staff. Gross motor items included catching a large ball, climbing the ladder of a slide and sliding down (not assessed), overhand throwing to a person $180 \mathrm{~cm}$ away, one-legged hopping, forward jumping $50 \mathrm{~cm}$, and balancing on one foot for $5 \mathrm{sec}$. Fine motor items included putting together a 5-7 piece puzzle, cutting 
paper in half with scissors along a straight line, drawing shapes $(\mathrm{L}+\mathrm{I}$ O), unbuttoning one or more buttons, drawing a picture of people with at least three features, and coloring in a 2-inch circle within the lines. "Yes" was scored when the child performed the skill consistently, "Sometimes" was scored when the skill was performed on occasion but inconsistently, and "No" was scored when the child was unable to perform the skill at all. A copy of the ASQ-3 fine and gross motor domains for 48 months can be found in APPENDIX 4.

\section{Executive Function}

Executive function is a composite of various cognitive capacities, such as working memory, inhibition, and shifting. ${ }^{188}$ Executive function is a contributor to self-regulatory development, ${ }^{189}$ a strong predictor of scholastic achievement, ${ }^{190}$ and a predictor of children's social and emotional development. ${ }^{191}$ The Early Years Toolbox (EYT; Early Start, Wollongong, NSW, Australia) is a battery of iPad games that assess visual-spatial and phonological working memory, shifting, inhibition, vocabulary, and parent-reported self-regulation and social behaviour. ${ }^{188}$ Each measure of the EYT was designed to be brief (less than 5 mins), engaging, developmentally appropriate, readily accessible, and able to leverage the capabilities of technology without introducing expertise bias. ${ }^{188}$ The EYT games also include visual and auditory instructions/feedback and practice trials to facilitate a clear understanding of the tasks in the games. Results from a study carried out by the creators of the EYT suggest that the battery of games have good reliability, convergent validity with existing measures, and developmental sensitivity. ${ }^{188}$ 
In this study, executive function of participants was assessed with the "Mr. Ant", "Go/No-Go", and "Card Sorting” EYT iPad games. Games were administered at the study sites and were supervised by a member of the research team. "Mr. Ant" was used to measure visual-spatial working memory and involved remembering the location of stickers placed randomly on the body of an ant. In the "Go/No-Go" game, which measured inhibition, participants were prompted to tap on fish that would swim across the screen and to do nothing when sharks would periodically swim across instead. Lastly, "Card Sorting", which measured shifting, asked participants to sort cards by a certain dimension (i.e., colour or shape) and then switch to an alternate sorting rule in the second half of the game. Members of the research team repeated game instructions and provided encouragement as necessary; however they refrained from providing excessive directives.

\section{Parent/Guardian and Centre/School Staff Feedback Forms}

To further inform the feasibility of the SUNRISE study, feedback questionnaires were distributed on the first day of testing, for parents/guardians and staff at centres/schools to complete on their own time, and were collected on the last day when accelerometers were retrieved from participants. Parent/guardian feedback questionnaires included four open-ended items related to their opinions on several aspects of the SUNRISE study (APPENDIX 5.). These parent/guardian feedback items included:

- "How did you find the parent/guardian questionnaire? Were there any questions that you didn't understand or found hard to answer?" 
- "Did your child enjoy participating? Was there anything you think they did not enjoy or that could be improved?"

- "How did you find it went for your child wearing the activity monitor for three days? Were there any problems? If so, can you tell us about them and offer any advice on how we could improve this?"

- "Do you have any other comments to make?"

Staff feedback questionnaires included five open-ended items concerning their thoughts on data collection within their centre/school (APPENDIX 6.). These staff feedback items included:

- "How did you find having the data collection team in your school/centre this week? Is there anything that you could recommend be done differently if the study was to be repeated to minimize any disruption to your daily routines?"

- "How do you think the children found the gross and fine motor skills activities and the iPad games? Do you have any feedback or advice on how the assessment of these items could be improved?"

- "Did the children enjoy participating? Was there anything you think they did not enjoy or that could be improved?"

- "How did you find it went for the children wearing the activity monitor for three days? Were there any problems? If so, can you tell us about them and offer any advice on how we could improve this?"

- "Do you have any other comments to make?" 


\section{Data Collection Protocol}

To follow the 3-weekday accelerometer wear protocol, the first day of data collection occurred on either a Monday or Tuesday so that the accelerometers could be retrieved on the corresponding Thursday or Friday of that week. Upon arrival at the study site on the first day of data collection, height and weight were measured and Acticals and activPALs were subsequently placed on participants. Participants were then given the option to choose the order in which they wanted to complete the gross motor testing, fine motor testing, and executive function testing. Each participant required roughly 30-45 mins to complete all testing measures, and research staff were able to work with multiple participants concurrently. After testing was completed, parent/guardian questionnaires were given to centre/school staff to send home with the participating children. In addition to the questionnaire, a document outlining how to wear the accelerometers and a parent/guardian feedback form were also sent home to the parents/guardians. Following the 3-days of accelerometer wear, research staff returned to the study site on the Thursday or Friday to collect accelerometers, parent/guardian questionnaires, and parent/guardian feedback forms from the participants.

\section{Accelerometer Data Reduction}

Actical data were reduced using in-house R code. Physical activity intensity was classified using validated cut-points for Acticals among preschoolers, with activity counts of $<25$ per $15 \mathrm{sec}$ equating to sedentary behaviour, $25-287$ per $15 \mathrm{~s}$ equating to LPA and activity counts $\geq 288$ per 15 s equaling MVPA. ${ }^{175}$ These cutpoints have been employed in previous analyses of Actical physical activity data from 
preschoolers. ${ }^{127,192}$ Step counts were also extracted from the raw Actical data. Nonwear time was defined as $\geq 60$ mins of consecutive zero activity counts, with the allowance for 2 mins of counts between $0-100 .{ }^{127}$ Sleep logs with wake and bed times were manually created for every day of each participant's data through visual inspection of the raw Actical data. These sleep logs were then applied during data reduction to exclude time spent sleeping at night from analyses of physical activity. The first and last days of Actical data were excluded from analyses since there were portions of those days where both research staff and the participants were in possession of the devices (i.e., application and removal of the devices). Valid days for analysis were defined as greater than $5 \mathrm{hrs}$ of wear time. ${ }^{167}$

activPAL data were reduced with custom MATLAB software (The MathWorks Inc., Natick, MA). Software algorithms identified sleep periods based on sleep diaries that were manually created based on visual inspection of the raw activPAL data. Due to a lack of validated cut-points, physical activity intensity was not extracted from the raw activPAL data, however step counts were still extracted. Similar to Actical analyses, valid days were defined as having greater than 5 hrs of wear time.

\section{Feasibility of the SUNRISE Study (Objective 1)}

Participants completed the SUNRISE study protocol to help identify potential issues with the protocol or measurement instruments. Feasibility of the SUNRISE study was partially assessed by the pilot study's ability to reach the target sample size of 100 participants, sex balanced with 50 from urban locations and 50 from rural locations in an efficient manner. In addition, feasibility of the study measures were 
assessed through the research staff, centre/school staff, participant's, and parent/guardian's capacity to follow the SUNRISE study protocol and effectively complete testing measures. The feasibility of measures was also informed through the observation of issues by members of the research staff during testing. Furthermore, the SUNRISE pilot study used responses from feedback questionnaires given to participating parents/guardians and centre/school staff to assess their opinions regarding participation and measures used in the study.

\section{4-Hour Movement Behaviour Adherence (Objective 2)}

Using the WHO Guidelines on Physical Activity, Sedentary Behaviour and Sleep for Children Under 5 Years of Age, ${ }^{2}$ movement behaviour data were analyzed for adherence to movement guidelines. Only participants with complete data for all movement behaviours were included in analyses of movement behaviour guideline adherence. ${ }^{127}$ Complete data was defined as 2 valid days of Actical data and the completion of the relevant sedentary behaviour and sleep items in the parent/guardian questionnaire.

\section{Physical Activity}

Actical physical activity data were used to assess adherence to physical activity guidelines, which recommend a total of 180 mins of physical activity, with at least 60 mins being MVPA, per day. ${ }^{2}$ Participants meeting both components of the physical activity recommendation were considered adherent to guidelines, and those meeting only one or none of the recommendations were considered non-adherent. 


\section{Sedentary Behaviour}

Data from the parent/guardian questionnaire were used to inform adherence to sedentary behaviour guidelines, which recommend no more than $1 \mathrm{hr}$ of sedentary screen time per day and not being restrained for more than $1 \mathrm{hr}$ at a time. ${ }^{2}$ Specifically, adherence to sedentary behaviour guidelines was determined with the following questions:

- "In a 24 hour period in the past week, how much time did the 4 year-old child who is participating in this study spend: Using any electronic screen device such as a smart phone, tablet, video game, or watch television or movies, videos on the internet while they were sitting or lying down?"

- "In the past week, on days that you spent with your child, were there any days where the 4 year-old child who is participating in this study: Was restrained for more than one hour at a time in a stroller, car seat, or on back or a scooter/moped?"

Participants meeting both sedentary behaviour recommendations were considered adherent to guidelines, while those who did not were considered nonadherent.

Sleep

Adherence to sleep guidelines, which recommend 10-13 hrs of good quality sleep (may include naps) with regular sleep and wake times, ${ }^{2}$ was also assessed with data from the parent/guardian questionnaire. The following questionnaire items were used to assess adherence to the guidelines: 
- "How many hours of sleep does your child get in a typical 24-hours day (including naps)?”

- "Does your child have a consistent bedtime?"

- "Does your child have a consistent wake-up time?"

If a child was meeting all three components of the sleep guidelines they were considered adherent. Not meeting one or more components of the sleep guidelines was considered non-adherent.

\section{Statistical Analyses}

One-way ANOVA tests were used to assess potential differences in the number of movement behaviour recommendations met based on participant characteristics (sex, weight status category, location). All analyses were carried out in R 3.5.2 (R Core Team, Vienna, Austria).

\section{International Screen Time Comparison (Objective 3)}

Screen time data from the SUNRISE pilot studies of eight countries (Brazil, Canada, China, Papua New Guinea, South Africa, Sweden, United States, and Zimbabwe) were shared by the University of Wollongong for analysis in this thesis project. The screen time data were extracted from the parent/guardian questionnaire item "In a 24 hour period in the past week, how much time did the 4 year-old child who is participating in this study spend: Using any electronic screen device such as a smart phone, tablet, video game, or watch television or movies, videos on the internet while they were sitting or lying down?". These data were used to examine potential differences between countries in the proportion of participants adhering to screen time recommendations and differences in the mean duration of daily screen time. 
Additional data received from the various pilot studies included participant sex, date of birth, date of data collection, highest household educational attainment, urban/rural status, average height and weight measurement, and BMI. Participants $\geq 5$ years-old were excluded from analyses because they no longer fell into the age range covered by movement guidelines for the early years.

The parent/guardian questionnaire item regarding highest household educational attainment was asked differently in each country's pilot study. As a result, the levels for this variable differed between countries, which necessitated recoding to be able to use the data in analyses. Responses were recoded, as necessary, into 5 levels generally representing no formal education or lowest level included, primary school or some high school, high school, post-secondary, and do not know/did not report.

\section{Statistical Analyses}

Screen time data were initially inspected for outliers and normality. Outliers were identified as values greater than $1.5 \cdot$ inter-quartile range $(I Q R)+3^{\text {rd }}$ quartile. ${ }^{193}$ Shapiro-Wilk tests and visual examination of histograms were used to assess the normality of the screen time variable. Countries were classified into three groups based on HDI: very high (0.8-1.0), high (0.7-0.79), and medium to low $(<0.69)$ HDI. The very high HDI group included Canada, Sweden, and the United States; the high HDI group included Brazil, China, and South Africa; and the medium to low HDI group included Papua New Guinea and Zimbabwe. Chi square tests were used to assess potential differences in guideline adherence between several participant characteristics (sex, weight category, urban/rural location, household educational 
attainment, country, and HDI). In addition, binary logistic regression models were used to assess associations between screen time adherence and sex, urban/rural location, and HDI while controlling for participant characteristics such as age, parental education, and sex. Spearman's rank correlation coefficient and KruskalWallis tests were also used to examine potential differences between daily screen time (mins) and these participant characteristics. Post-hoc pairwise comparisons were performed when tests were significant and the independent variable was composed of greater than 2 levels. Post-hoc tests were Bonferroni corrected to reduce the chance of Type I errors.

\section{Comparison of Actical and activPAL Physical Activity, Sedentary}

\section{Behaviour, and Sleep Data (Objective 4)}

Actical and activPAL data from participants who wore both devices simultaneously were compared for differences in the measurement of movement behaviours. Step counts and time spent sitting/lying down (sedentary time) recorded by activPALs and Acticals were compared to assess potential measurement biases. For the purpose of these calculations, deviations of activPAL outcomes from Actical outcomes were calculated for each day where both devices had a valid day of data. To improve comparability, step counts and sedentary/sitting time were weighted by wear time (mins), which was calculated for each device during data reduction.

\section{Statistical Analyses}

Paired t-tests were used to assess potential differences in weighted step counts and sedentary/sitting time recorded by Acticals and activPALs. Mean measurement differences were also calculated for weighted steps and sedentary/sitting time. 
Additionally, Bland-Altman plots were generated to visually assess potential steps and sedentary/sitting time measurement biases between both devices. Lastly, single fixed rater intraclass correlation coefficients (ICC) were calculated to assess the reliability between weighted measures of steps and sedentary/sitting time as measured by Acticals and activPALs. 


\section{Results}

A total of 118 children consented to participate in the Canadian SUNRISE pilot study between July 2018 and May 2019. Of those who consented, 106 (Female = 51 , Male $=55)$ participants completed testing. Twenty-three participants were from rural locations and 83 were from urban locations. Fifteen participants were recruited from childcare centres, and the remaining 91 participants were recruited from schools. Descriptive characteristics of 102 participants with completed parent/guardian questionnaires are presented in Table 1. 
Table 1. Descriptive characteristics of participants in the Canadian SUNRISE pilot study.

\begin{tabular}{|c|c|}
\hline Variable & $(n=102)^{a}$ \\
\hline Age (years) & $4.5 \pm 0.5$ \\
\hline \multicolumn{2}{|l|}{ Sex } \\
\hline Male & $53.9 \%(n=55)$ \\
\hline Female & $46.1 \%(n=47)$ \\
\hline \multicolumn{2}{|l|}{ Highest household educational attainment } \\
\hline Post-secondary & $98.0 \%(n=100)$ \\
\hline Secondary or high school & $1.0 \%(\mathrm{n}=1)$ \\
\hline Unanswered & $1.0 \%(\mathrm{n}=1)$ \\
\hline \multicolumn{2}{|l|}{ Ethnicity } \\
\hline Arab & $6.9 \%(n=7)$ \\
\hline Asian & $3.9 \%(n=4)$ \\
\hline Black & $3.9 \%(n=4)$ \\
\hline Latin American & $2.9 \%(n=3)$ \\
\hline Mixed & $11.8 \%(n=12)$ \\
\hline White & $70.6 \%(n=72)$ \\
\hline \multicolumn{2}{|l|}{ BMI z-score weight category ${ }^{b}$} \\
\hline Normal-weight & $74.5 \%(\mathrm{n}=76)$ \\
\hline At-risk of overweight & $18.6 \%(\mathrm{n}=19)$ \\
\hline Overweight & $6.9 \%(n=7)$ \\
\hline Mean BMI z-score & $0.6 \pm 0.9$ \\
\hline \multicolumn{2}{|l|}{ Recruitment site } \\
\hline Childcare centre & $14.7 \%(n=15)$ \\
\hline School & $85.3 \%(\mathrm{n}=87)$ \\
\hline \multicolumn{2}{|l|}{ Location } \\
\hline Urban & $78.4 \%(n=80)$ \\
\hline Rural & $21.6 \%(n=22)$ \\
\hline
\end{tabular}

${ }^{a}$ Mean and standard deviation values were presented for continuous variables, and percentage and frequency for categorical variables.

${ }^{\mathrm{b}}$ Body mass index (BMI) z-scores were categorized using the following definitions: wasted $\mathrm{z}$-score $=<-2.0$; normal-weight $\mathrm{z}$-score $=\geq-2.0$ and $\leq 1.0$; at-risk of overweight $=>1.0$ and $\leq 2.0 ;$ overweight $=>2.0$ and $\leq 3.0$; obese $=>3.0$.

\section{Feasibility of the SUNRISE Study (Objective 1)}

\section{Research Ethics}

Research ethics approval for this study was received from the CHEO REB in June 2018. This initial research ethics submission specified data collection at childcare centres only, which was aligned with the original SUNRISE pilot protocol. 
Data collection at childcare centres occurred between July 2018 and August 2018.

However, due to the commencement of the school year in September, most 4 year-old children switched from attending childcare centres to attending schools for their first year of kindergarten. In response to this change, a minor amendment was submitted to the CHEO REB and a research ethics application was submitted to the OCREAC to allow for recruitment within the public-school boards in Ottawa. Another research ethics application was made to the Upper Canada District School Board (UCDSB) External Research Steering Committee. The UCDSB is the school board that services the broad rural communities surrounding the City of Ottawa. Participants from the UCDSB were intended to accommodate our rural target sample size, however the study's research ethics application was declined by the UCDSB. It was explained by the Steering Committee that the study was not approved due to a lack of direct benefits that the research would provide to the UCDSB. The study's research ethics application to the OCREAC was approved in October 2018 and data collection was carried out at schools from November 2018 to May 2019. Research ethics approval was received from the CUREB in September 2018.

\section{Site Recruitment}

Twenty-three childcare centres in and around the Ottawa-area were contacted to solicit their participation in the study. Of the centres contacted, $4(17.4 \%)$ agreed to allow recruitment within their sites. Out of the 145 eligible children at these centres, 20 children consented to participate in the study and 15 of these children completed study testing. The final enrollment rate of eligible children at childcare centres was $10.3 \%$. 
All schools in the OCDSB and OCSB $(n=169)$ were contacted about their willingness to participate in the study. Recruitment was allowed within $12(7.1 \%)$ of the contacted schools, and out of 513 eligible children, 98 children consented to participate in the study. Ninety-one children completed testing, which equated to an enrollment rate of $17.7 \%$ of eligible children within schools that provided approval for recruitment.

\section{Target Sample}

The overall target sample size of 100 participants was met in the Canadian SUNRISE pilot study. One hundred and six participants were recruited and completed testing in the study. The sample was also sex balanced with 51 female and 55 male participants. In addition, 83 participants from urban locations completed study testing, which exceeded the target sample of 50 urban participants. However, only 23 participants from rural locations completed study testing, which did not meet the target sample size of 50 rural participants. Seven of the rural participants were recruited from childcare centres and 16 were from schools.

\section{Withdrawals and Exclusions}

Two participants were withdrawn from the study after refusing to wear their accelerometers on the first day of testing. As well, 10 participants were excluded for being over-age or absent at the time of data collection. Participating childcare centres and schools were visited on multiple occasions in an attempt to include previously absent children who had consented to participation in the study. 


\section{Feasibility of Accelerometry}

Overall, participants had relatively good adherence to the accelerometer protocol. Of the 106 participants that completed testing, $85 \%$ completed the accelerometer protocol with 2 valid days of data. Application of Acticals was quick and non-intrusive. One Actical was lost by a participant and was not able to be retrieved. In feedback forms, $15 \%(\mathrm{n}=16)$ of parents commented about belts being too large, being uncomfortable, or falling off for their child. In addition, $10 \%(\mathrm{n}=11)$ of parents commented on their child wanting to remove their accelerometer at some point during the data collection period. It was also reported by $3 \%(n=3)$ of parents that they forgot to put their child's Actical back on after taking it off for water-based activities. Reduction of Actical data was relatively simple due to well-established activity count cut-points for categorizing physical activity intensities. However, delineating sleep from waking hours was a time-consuming component of Actical data reduction and required the manual creation of sleep logs for each day of data through the visual examination of the raw activity count data. Acticals have not been validated for objectively measuring sleep in preschool-aged children and therefore sleep periods were excluded from analyses.

There were several issues related to the use of activPALs in the study. First, application of the devices was difficult and time-consuming when participants were wearing pants or leggings because the protocol called for the devices to be placed on the mid-anterior thigh. During the summer, most children were wearing shorts and skirts and the devices could be easily applied on their thighs. However, during the colder months (Fall to Spring), most children were wearing pants or leggings, which 
required the child to change into a pair of shorts or a member of the research staff to accompany each child to the bathroom to apply the device in private. In addition, despite being waterproofed prior to data collection, one activPAL was permanently water damaged while being worn by a participant. There were also no standards or cut-points for reducing the activPAL data of preschool-aged children, therefore physical activity analyses were limited to step counts only. Lastly, as a result of four participants reporting skin irritation associated with wearing activPALs, the devices were removed from the study protocol. In total, 33 participants wore activPALs before they were removed from the study. In spite of the issues with activPALs, many participants enjoyed wearing the devices and spoke fondly of their 'robots' and the 'superpowers' that they gave them. Actical devices remained in the study protocol throughout the entire study.

The 3-day accelerometer wear protocol was problematic as implemented. It was not clearly outlined if the 3-days referred to 72-hrs of device wear time or if weekdays and weekends were eligible, or required. As a result, the decision was made to only collect weekdays and to only have participants wear the accelerometers for approximately 72-hrs from the time that the device was applied to reduce the burden on the participants and parents. Given that participants were not in possession of the devices for the full 24-hour period on the days where the devices were applied and removed, data from these days were not included in analyses. Thus, the " 3 -day protocol" limited the number of valid days available for analyses to a maximum of 2 days. 


\section{Feasibility of Motor Skills}

Participants generally enjoyed gross and fine motor skills testing from the ASQ, although several issues emerged during the study. The "climbing up and sliding down a slide" item from the gross motor skills questionnaire was not scored for any participants because there was a lack of readily accessible slides at study sites. Additionally, scoring fine and gross motor skills was sometimes difficult to discern between scoring items as "no" or "sometimes". There was no standardization of tools used for ASQ testing, such as ball size, specified in the study protocol manual. Therefore, materials used for ASQ testing were likely different across pilot countries. The ASQ was also noted as being designed as a parent-administered questionnaire and was not intended to be applied by researchers in a research-context.

\section{Feasibility of Executive Function}

Participants generally found the EYT games to be repetitive, monotonous, and excessively long. Participants often requested to switch to a different game midway through the EYT games. Additionally, participants would often ask "Why do they keep saying that?" in response to the repetitive auditory prompts of the games.

Research staff had to actively supervise and encourage participants to remain focused and to keep playing the EYT games. During the "Go/No-Go" game, children often became distracted and failed to attempt several rounds of fishes/sharks swimming across the screen, which may have influenced their scores on the game. In addition, some participants purposely played the "Mr. Ant" game improperly by arbitrarily placing stickers all over the ant character instead of placing stickers based on their memory. The "Card Sort" game was completed correctly by most participants; 
although there were similar issues with participants remaining engaged with the game.

\section{Feasibility of Parent/Guardian Questionnaire}

Of the 106 participants that completed testing, 96\% of parents/guardians completed and returned the study questionnaire. Several issues with the parent/guardian questionnaire items were identified during pilot testing. One group of questions beginning with the prompt "In a 24 hour period in the past week, how much time did the 4 year-old child who is participating in this study spend: " was at times misinterpreted by parents/guardians due to the way it was worded. Instead of responding with time from a 24-hour period, many parents/guardians responded with the total time in the past week (e.g., 14 hrs per week instead of 2 hrs per day). In feedback forms, $7 \%(\mathrm{n}=7)$ of parents commented that the wording "In a 24 hour period in the past week" was confusing and awkward. In addition, 15\% $(\mathrm{n}=16)$ of parents commented that times spent in various activities were very approximate since they were not completely certain of their child's activities when at childcare or school. Furthermore, $15 \%(\mathrm{n}=16)$ of parents indicated that their child's activity varied significantly between the winter and summer months. Some parents $(8 \%, n=$

9) also commented that questions referring to the "Main Caregiver" in the household were confusing, as both parents in their households equally shared caregiving responsibilities for their children. As well, a block of questions was consistently left blank due to an error in the questionnaire. A conditional question that gave instructions to skip several questions if a "No" was answered, was mislabeled and accidentally instructed the user to skip a greater number of questions than was 
intended. Lastly, parent/guardian questionnaires that were not returned limited the analyses that could be carried out with their child's data. Critical information related to the participant's date of birth, sedentary behaviour, and sleep were included in the parent/guardian questionnaire. Therefore, when questionnaires were not returned, analyses requiring these data were not possible.

\section{Staff Feedback Questionnaires}

Staff unanimously responded in their feedback questionnaires that having researchers in their sites was not disruptive to their daily flow of activities. Staff also reported that their children seemed to enjoy participating in the study. Staff reported that they found the accelerometer protocol was okay but commented that Actical belts needed adjusting because they would become loose at times. However, these reports were based on only 3 completed staff questionnaires.

\section{Proportion of Children Adhering to 24-Hour Movement Guidelines}

\section{(Objective 2)}

\section{Participant Sample for Analyses}

Eighty-six participants with 2 valid days of Actical data and complete parent/guardian questionnaire data were included in analyses of 24-hour movement guideline adherence. The mean age of participants was $4.5( \pm 0.5)$ years and their mean BMI z-score was $0.7( \pm 0.9)$. Participants engaged in an average of 383 mins of daily total physical activity, of which an average of 67 mins was MVPA. Participants slept for an average duration of 10.5 hrs daily and had an average of 94 mins of daily screen time. Descriptive characteristics and time spent in 24-hour movement behaviours of study participants are presented in Tables 2 and 3, respectively. 


\section{Adherence to Guidelines}

Overall, $19.8 \%$ of participants met all movement behaviour recommendations, $48.8 \%$ met two recommendations, $25.6 \%$ met one recommendation, and $5.8 \%$ met no recommendations from the WHO's Guidelines on Physical Activity, Sedentary Behaviour and Sleep for Children Under 5 Years of Age. ${ }^{2}$ Sleep recommendations were most frequently met (76.7\%), and approximately half of participants met the physical activity (55.8\%) and sedentary behaviour (50.0\%) recommendations. Adherence to movement behaviour recommendations, individually and in combination, are presented in a Venn diagram in Figure 1. The mean number of recommendations met by sexes was 1.9 for males and 1.7 for females, which were not significantly different based on one-way ANOVA tests $(\mathrm{F}(1,84)=1.041, p=.31)$. The mean number of recommendations met for normal-weight, at-risk of overweight, and overweight categories was $1.9,1.7$, and 1.6 , respectively. These weight category differences were not statistically significant $(\mathrm{F}(2,83)=0.7567, p=.47)$. Participants from urban locations met a mean of 1.8 recommendations and those from rural locations met a mean of 2.0, which were not statistically different $(\mathrm{F}(1,84)=2.092, p$ $=.15)$. 
Table 2. Descriptive characteristics of participants included in analyses of movement guideline adherence in the Canadian SUNRISE pilot study.

\begin{tabular}{|c|c|}
\hline Variable & $(n=86)^{a}$ \\
\hline Age (years) & $4.5 \pm 0.5$ \\
\hline \multicolumn{2}{|l|}{ Sex } \\
\hline Male & $52.3 \%(\mathrm{n}=45)$ \\
\hline Female & $47.7 \%(n=41)$ \\
\hline \multicolumn{2}{|l|}{ BMI z-score weight category ${ }^{b}$} \\
\hline Normal-weight & $73.3 \%(n=63)$ \\
\hline At-risk of overweight & $18.6 \%(n=16)$ \\
\hline Overweight & $8.1 \%(n=7)$ \\
\hline Mean BMI z-score & $0.7 \pm 0.9$ \\
\hline \multicolumn{2}{|l|}{ Recruitment site } \\
\hline Childcare centre & $16.3 \%(n=14)$ \\
\hline School & $83.7 \%(\mathrm{n}=72)$ \\
\hline \multicolumn{2}{|l|}{ Location } \\
\hline Urban & $75.6 \%(n=65)$ \\
\hline Rural & $24.4 \%(n=21)$ \\
\hline
\end{tabular}

${ }^{a}$ Mean and standard deviation values were presented for continuous variables, and percentage and frequency for categorical variables.

${ }^{\mathrm{b}}$ Body mass index (BMI) z-scores were categorized using the following definitions: wasted $\mathrm{z}$-score $=<-2.0$; normal-weight $\mathrm{z}$-score $=\geq-2.0$ and $\leq 1.0$; at-risk of overweight $=>1.0$ and $\leq 2.0$; overweight $=>2.0$ and $\leq 3.0$; obese $=>3.0$.

Table 3. Daily time Canadian SUNRISE pilot study participants spent in total physical activity, moderate to vigorous intensity physical activity, screen time, and sleep.

\begin{tabular}{|c|c|c|}
\hline \multicolumn{2}{|c|}{} & Full Sample (n=86) \\
\hline Daily time in movement behaviours & Mean $\pm \mathbf{S D}^{\mathbf{a}}$ & $\mathbf{9 5 \%} \mathbf{C I}^{\mathbf{b}}$ \\
\hline Total physical activity (mins) & $384.6 \pm 71.7$ & $397.9-367.6$ \\
Moderate to vigorous physical activity & $66.8 \pm 27.4$ & $72.5-61.0$ \\
(mins) & & \\
Screen time (mins) & $94.0 \pm 93.9$ & $113.8-74.1$ \\
Sleep (hrs) & $10.5 \pm 1.0$ & $10.7-10.3$ \\
\hline
\end{tabular}

${ }^{\text {a }}$ Standard deviation (SD)

${ }^{\mathrm{b}}$ Confidence interval (CI) 


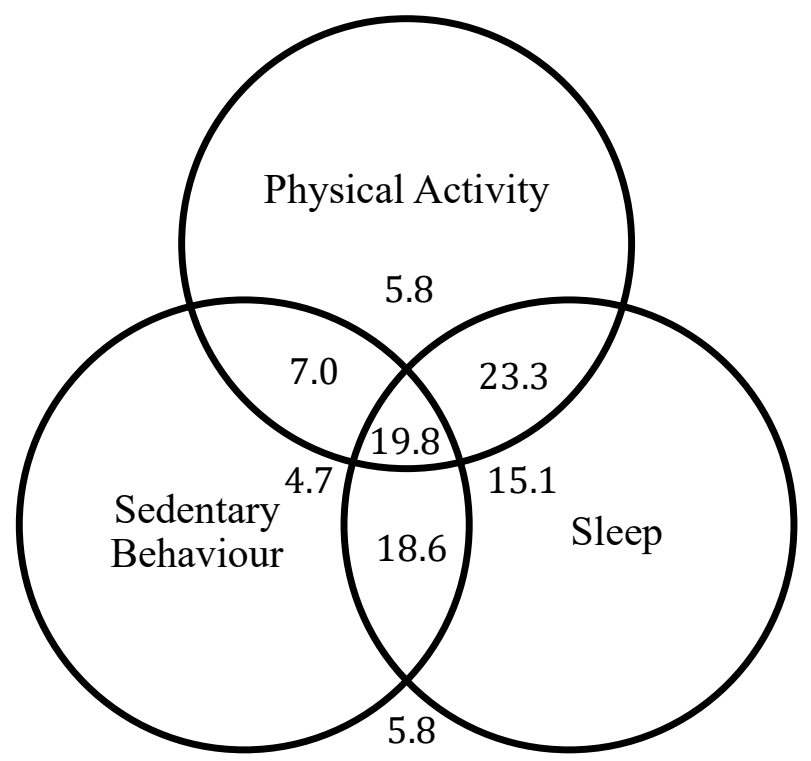

Figure 1. Venn Diagram of the proportion (\%) of participants $(n=86)$ meeting no recommendations; physical activity, sedentary behaviour, and sleep recommendations; and combinations of recommendations.

Legend: The respective recommendations for physical activity, sedentary behaviour, and sleep: $\geq 180$ mins of daily physical activity with at least 60 mins being moderate to vigorous intensity; no more than $1 \mathrm{hr}$ of daily screen time and not restrained while seated for more than $1 \mathrm{hr}$; and 10-13 hrs of daily sleep with consistent sleep and wake times. The sum of each circle equals the proportions of participants meeting recommendations for that individual movement behaviour.

\section{International Screen Time Comparison (Objective 3)}

Data from 797 participants from the Brazil, Canada, China, Papua New Guinea, South Africa, Sweden, United States, and Zimbabwe SUNRISE pilot studies were available for analyses. These countries represented a range of HDI backgrounds from a high of 0.937 in Sweden to a low of 0.543 in Papua New Guinea. The overall sample included participants ranging in age from 2.6-5.9 years-old. Participants not between 3.00-4.99 years of age, and participants missing screen time or anthropometric data were excluded from analyses $(n=206)$. Consequently, 591 participants were included in analyses, with a mean age of $4.3 \pm 0.4$ and a mean BMI z-score of $0.6 \pm 1.2$. Descriptive characteristics of participants are presented in Table 
4 and mean and median screen time per country are presented in Table 5. ShapiroWilk normality tests and visual inspection of histograms indicated that the screen time data were not normally distributed; therefore non-parametric tests were used for analyses. In total, 21 daily screen time outliers were identified, however sensitivity analyses revealed that removing these participants (3.6\% change in sample size) resulted in a change of only $1.9 \%$ in terms of participants meeting screen time recommendations. The screen time outlier values were all plausible and it was not possible to determine whether they were the result of error, so they were left in the sample for analysis.

Overall, participants averaged $99.8 \pm 91.3$ mins (median $=60$ mins) of screen time per day. Mean daily screen time ranged from a high of $158 \pm 125$ mins (median $=120$ mins $)$ in Brazil to a low of $70 \pm 82$ mins (median $=30$ mins $)$ in Papua New Guinea. In the overall sample, $51 \%$ of participants met screen time recommendations. Among the urban and rural samples, $52 \%$ and $50 \%$ of participants met recommendations, respectively. The Brazilian sample had the lowest screen time adherence with only $20 \%$ meeting recommendations, whereas China and Papua New Guinea lead all countries with $66 \%$ adherence. Country HDI levels and proportion of participants meeting recommendations by country, sex, and location can be found in Table 6.

Results from Chi square tests showed that there were statistically significant differences between countries in the proportion of participants meeting screen time guidelines $\left(\chi^{2}(7, \mathrm{n}=591)=49.3, p<.001\right)$. Post-hoc pairwise comparison of proportion tests revealed several significant differences in the proportion of 
participants meeting screen time recommendations between several countries, as presented in Table 7. Chi square test results also indicated that there were no statistically significant screen time adherence differences between sexes $\left(\chi^{2}(1, n=\right.$ $591)=0.38, p=.54)$ or between participants from urban and rural areas $\left(\chi^{2}(1, \mathrm{n}=\right.$ $591)=0.36, p=.55$ ). Chi square tests were statistically significant for adherence differences between BMI z-score categories $\left(\chi^{2}(4, \mathrm{n}=591)=10.7, p=.03\right)$, however Bonferroni adjusted pairwise comparison of proportion tests did not indicate any statistically significant category comparisons. Additionally, chi square tests indicated statistically significant differences between highest household educational attainment categories and guideline adherence $\left(\chi^{2}(4, \mathrm{n}=591)=11.8, p=.02\right)$, although differences were only significant between the second (primary school/some high school) and third (high school) categories ( $p=.03)$.

Logistic regression results and odds ratios for HDI group, sex, and urban/rural location associated with meeting/not meeting screen time recommendations are presented in Table 8. Odds ratios were not calculated for weight categories due to small group sizes. When controlling for age, sex, and parental education, odds ratios were not statistically significant for urban/rural locations. When controlling for age and parental education, the odds ratio for sex was not statistically significant. Tukeycorrected odds ratios were statistically significant for the very high relative to the high HDI group (odds ratio $=0.62, p=.04$ ) and the medium to low relative to the very high HDI group (odds ratio $=2.74, p<.001$ ).

Spearman's Rank Correlation Coefficient test results indicated that daily screen time was not significantly associated with BMI z-scores $\left(r_{s}(589)=.08, p=\right.$ 
.06 ,) or age $\left(r_{s}(589)=.03, p=.50\right)$. Kruskal-Wallis tests similarly indicated no differences in daily screen time between sexes $\left(\chi^{2}(1, \mathrm{n}=591)=0.05, p=.83\right)$ or between urban and rural participants $\left(\chi^{2}(1, \mathrm{n}=591)=0.52, p=.47\right)$. Kruskal-Wallis tests revealed significant differences in screen time levels between parental education categories $\left(\chi^{2}(4, \mathrm{n}=591)=25.8, p<.001\right)$. Bonferroni adjusted pairwise comparisons showed that screen time differences were significant between the primary school/some high school and high school $(p<.001)$, primary school/some high school and post-secondary $(p=.02)$, and high school and post-secondary levels $(p=.03)$.

Chi square tests revealed statistically significant differences in proportions adhering to screen time recommendations between HDI groups $\left(\chi^{2}=12.4, p=.002\right)$. Pairwise comparison of proportions indicated that this difference was between the very high HDI and medium to low HDI groups ( $p=.003$ ). Similarly, Kruskal-Wallis tests revealed significant differences in daily screen time between HDI groups $\left(\chi^{2}(2, n\right.$ $=591)=24.3, p<.001$ ), with pairwise comparisons of proportions indicating significantly higher screen time in the very high HDI group relative to the medium to low HDI group $(p=.002)$. Daily screen time and screen time recommendation adherence broken down by HDI groups can be seen in Figures 2 and 3, respectively. 
Table 4. Descriptive characteristics of participants from the SUNRISE pilot studies of 8 countries included in analyses of screen time adherence.

\begin{tabular}{|c|c|}
\hline Variable & Full sample $(\mathrm{n}=591)^{\mathrm{a}}$ \\
\hline Age (years) & $4.3 \pm 0.4$ \\
\hline \multicolumn{2}{|l|}{ Sex } \\
\hline Male & $50.3 \%(n=297)$ \\
\hline Female & $49.7 \%(n=294)$ \\
\hline \multicolumn{2}{|l|}{ BMI z-score weight category ${ }^{b}$} \\
\hline Wasting & $0.7 \%(n=4)$ \\
\hline Normal-weight & $69.0 \%(n=408)$ \\
\hline At-risk of overweight & $21.5 \%(n=127)$ \\
\hline Overweight & $6.6 \%(n=39)$ \\
\hline Obese & $2.2 \%(n=13)$ \\
\hline Mean BMI z-score & $0.6 \pm 1.2$ \\
\hline \multicolumn{2}{|l|}{ Location } \\
\hline Urban & $51.9 \%(n=307)$ \\
\hline Rural & $48.1 \%(n=284)$ \\
\hline \multicolumn{2}{|l|}{$\begin{array}{l}\text { Highest household educational } \\
\text { attainment }\end{array}$} \\
\hline No schooling/Lowest level & $0.2 \%(\mathrm{n}=1)$ \\
\hline Primary school/Secondary School & $9.5 \%(n=56)$ \\
\hline High School & $32.5 \%(n=192)$ \\
\hline Post-secondary & $55.0 \%(n=325)$ \\
\hline Do not know/Did not answer & $2.7 \%(n=16)$ \\
\hline
\end{tabular}

${ }^{a}$ Mean and standard deviation values were presented for continuous variables, and percentage and frequency for categorical variables.

${ }^{\mathrm{b}}$ Body mass index (BMI) z-scores were categorized using the following definitions: wasted z-score $=<-2.0$; normal-weight $\mathrm{z}$-score $=\geq-2.0$ and $\leq 1.0$; at-risk of overweight $=>1.0$ and $\leq 2.0 ;$ overweight $=>2.0$ and $\leq 3.0$; obese $=>3.0$.

Table 5. Mean and median daily screen time among SUNRISE pilot study participants from 8 countries.

\begin{tabular}{|c|c|c|}
\hline Country (Sample size) & \multicolumn{2}{|c|}{ Daily Screen Time (mins) } \\
\hline & Mean \pm SD $^{\mathbf{a}}$ & ${\text { Median }(\mathbf{Q R})^{\mathbf{b}}}^{\mathbf{b}}$ \\
Brazil $(\mathrm{n}=49)$ & $158.0 \pm 124.7$ & $120(90)$ \\
Canada $(\mathrm{n}=99)$ & $98.4 \pm 95.4$ & $60(60)$ \\
China $(\mathrm{n}=141)$ & $75.9 \pm 66.6$ & $60(90)$ \\
Papua New Guinea $(\mathrm{n}=80)$ & $69.9 \pm 81.8$ & $120(105)$ \\
South Africa $(\mathrm{n}=81)$ & $127.2 \pm 105.8$ & $90(60)$ \\
Sweden $(\mathrm{n}=94)$ & $104.8 \pm 69.3$ & $120(135)$ \\
United States $(\mathrm{n}=27)$ & $118.3 \pm 93.4$ & $52.5(123.75)$ \\
Zimbabwe $(\mathrm{n}=20)$ & $93.3 \pm 94.4$ & $60(90)$ \\
Overall $(\mathrm{n}=591)$ & $99.9 \pm 91.3$ & \\
\hline
\end{tabular}

${ }^{a}$ Standard deviation (SD)

${ }^{\mathrm{b}}$ Interquartile range (IQR) 
Table 6. Proportion of participants in 8 SUNRISE pilot studies adhering to screen time recommendations (no more than $1 \mathrm{hr}$ of daily screen time) by country, sex, location, and highest household educational attainment.

\begin{tabular}{|c|c|}
\hline Variable & $\begin{array}{c}\text { Proportion Adhering to Screen Time } \\
\text { Recommendations (\%) }\end{array}$ \\
\hline Country (HDI) & \\
Brazil $(0.761)$ & 20.4 \\
Canada (0.922) & 51.5 \\
China (0.758) & 66.0 \\
Papua New Guinea (0.543) & 66.3 \\
South Africa (0.705) & 48.1 \\
Sweden (0.937) & 37.2 \\
United States (0.920) & 33.3 \\
Zimbabwe (0.563) & 55.0 \\
\hline HDI Group & \\
Very high (0.8-1.0) & 43.2 \\
High (0.7-0.79) & 52.4 \\
Medium to low (<0.69) & 64.0 \\
\hline Sex & 52.2 \\
Male & 49.7 \\
Female & 52.1 \\
\hline Location & 49.6 \\
Urban & \\
Rural & 100 \\
\hline attainment & 66.1 \\
Highest houseld educational & 42.7 \\
No schooling/Lowest level & 52.6 \\
Primary school/some high school & 56.3 \\
High School & \\
Post-secondary & \\
Do not know/Did not answer & \\
\hline
\end{tabular}

${ }^{\mathrm{a}}$ Human Development Index (HDI) 
Table 7. P-value results of post-hoc pairwise comparison of proportions of participants meeting screen time guidelines ( $\leq 1 \mathrm{hr}$ daily screen time) by SUNRISE pilot study country.

\begin{tabular}{|c|c|c|c|c|c|c|c|}
\hline & Brazil & Canada & China & $\begin{array}{c}\text { Papua } \\
\text { Guinea }\end{array}$ & $\begin{array}{c}\text { South } \\
\text { Africa }\end{array}$ & Sweden & $\begin{array}{c}\text { United } \\
\text { States }\end{array}$ \\
\hline Canada & 0.02 & - & - & - & - & - & - \\
\hline China & $<.001$ & 0.9 & - & - & - & - & - \\
\hline $\begin{array}{c}\text { Papua New } \\
\text { Guinea }\end{array}$ & $<.001$ & 1.0 & 1.0 & - & - & - & - \\
\hline $\begin{array}{c}\text { South } \\
\text { Africa }\end{array}$ & 0.08 & 1.0 & 0.39 & 0.86 & - & - & - \\
\hline Sweden & 1.0 & 1.0 & $<.001$ & 0.007 & 1.0 & - & - \\
\hline $\begin{array}{c}\text { United } \\
\text { States }\end{array}$ & 1.0 & 1.0 & 0.08 & 0.16 & 1.0 & 1.0 & - \\
\hline Zimbabwe & 0.31 & 1.0 & 1.0 & 1.0 & 1.0 & 1.0 & 1.0 \\
\hline
\end{tabular}

Table 8. Binary logistic regression analysis of screen time recommendation $(<1 \mathrm{hr}$ daily screen time) adherence by Human Development Index, sex, and urban/rural location based on SUNRISE pilot studies in 8 countries (Brazil, Canada, China, Papua New Guinea, South Africa, Sweden, United States, and Zimbabwe; $\mathrm{n}=591$ ).

\begin{tabular}{|c|c|c|c|c|}
\hline Predictor & $\beta^{\mathrm{a}}$ & $\begin{array}{c}\text { Standard } \\
\text { Error } \beta\end{array}$ & $p$-value & $\begin{array}{c}e^{\beta} \\
\text { (odds } \\
\text { ratio) }\end{array}$ \\
\hline \multicolumn{5}{|l|}{$\mathrm{HDI}^{\mathrm{b}}$} \\
\hline $\begin{array}{r}\text { Medium to low }(<0.69)- \\
\text { High }(0.70-0.79)\end{array}$ & 0.54 & 0.25 & .08 & 1.72 \\
\hline Very high $(0.8-1.0)$ - High & -0.47 & 0.20 & .04 & 0.62 \\
\hline Medium to low - Very high & 1.01 & 0.28 & $<.001$ & 2.74 \\
\hline \multicolumn{5}{|l|}{$\operatorname{Sex}^{\mathrm{c}}$} \\
\hline Male - Female & -0.09 & 0.16 & .57 & 0.91 \\
\hline Urban/rural location $^{\mathrm{d}}$ & -0.11 & 0.17 & .50 & 0.89 \\
\hline
\end{tabular}

${ }^{a}$ Beta coefficient

${ }^{\mathrm{b}}$ Model controlling for age, sex, and parental education; Human Development Index (HDI); very high (Canada, Sweden, and United States), high (Brazil, China, and South Africa), and medium to low (Papua New Guinea and Zimbabwe)

${ }^{\mathrm{c}}$ Model controlling for age and parental education.

${ }^{\mathrm{d}}$ Model controlling for age, sex, and parental education. 


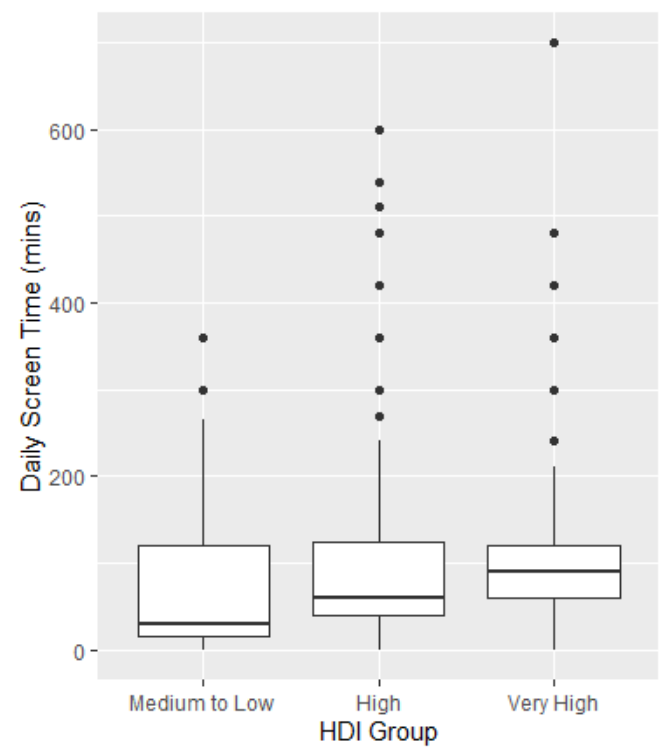

Figure 2. Box plots of daily screen time among SUNRISE pilot study participants (n = 591) categorized into very high (Canada, Sweden, and United States), high (Brazil, China, and South Africa), and medium to low (Papua New Guinea and Zimbabwe) Human Development Index (HDI) categories.

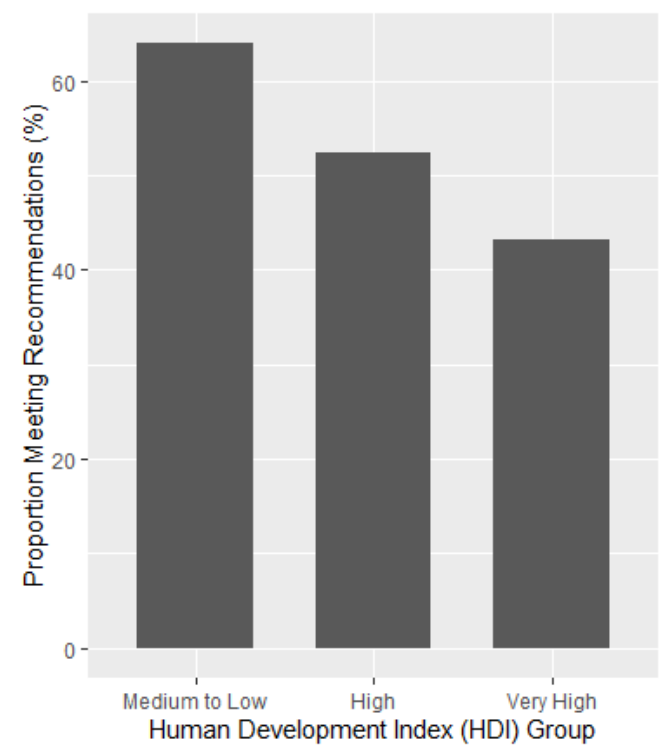

Figure 3. Bar plot of adherence to screen time recommendations (no more than $1 \mathrm{hr}$ of daily screen time) by very high (Canada, Sweden, and United States), high (Brazil, China, and South Africa), and medium to low (Papua New Guinea and Zimbabwe) Human Development Index (HDI) categories among SUNRISE pilot study participants $(\mathrm{n}=591)$. 


\section{Actical and activPAL Movement Behaviour Measurement Comparison}

\section{(Objective 4)}

There were 31(12 female, 19 male) participants with activPAL and Actical data available for analyses, with 58 total days of accelerometer wear time available for comparison of steps and sedentary/sitting time. Descriptive characteristics of participants included in analyses are presented in Table 9. The mean wear time for Acticals was $803 \pm 70$ mins per day and $836 \pm 2$ mins per day for activPALs. Mean daily steps recorded by Actical and activPALs were 11,340 \pm 4497 and 10,911 \pm 3664, respectively. Mean sedentary/sitting time was $440 \pm 88$ mins per day as measured by Acticals and $467 \pm 130$ mins per day as measured by activPALs. Daily steps and sedentary/sitting time were weighted by wear time calculated for that day.

Data from Acticals and activPALs were compared with paired t-tests. Step counts alone ( $\mathrm{t}=0.783 \mathrm{df}=57, \mathrm{p}=.44)$ and step counts weighted by wear time $(\mathrm{t}=1.836$, $\mathrm{df}=57, \mathrm{p}=.07)$ were not significantly different, with mean differences of 429 steps and 1 step per min of wear time, respectively. Sedentary time recorded by Acticals and sitting time recorded by activPALs alone had a mean difference of -26.7 mins and were not significantly different $(\mathrm{t}=-1.555, \mathrm{df}=57, \mathrm{p}=.13)$. Wear time weighted sedentary/sitting time had a mean difference of -0.005 mins of sedentary/sitting time per min of wear time, which was not statistically significant $(\mathrm{t}=-0.400, \mathrm{df}=57, \mathrm{p}=.69)$.

Bland-Altman plots comparing weighted steps and sedentary/sitting time between devices were generated and are presented in Figures 4 and 5, respectively. The mean difference between weighted measures of steps was 1.15 , indicating a bias of +1.15 steps per min of wear time with activPALs relative to Acticals. Visually, 
from the steps plots, it appears that Acticals tend to measure fewer steps than activPALs when the average steps per min of wear time are lower and the opposite trend was true when the average steps per min of wear time was higher. In the weighted steps plots, no values laid outside of the $95 \%$ CIs. In terms of the weighted sedentary/sitting time plots, the mean measurement difference was -0.0047 , which indicates a bias of -0.28 seconds of sedentary/sitting time per min of wear time among activPALs relative to Acticals. Values appeared equally distributed in the sedentary/sitting time plots and only one value was outside of the $95 \%$ CIs.

The computed ICC for weighted steps showed that the reliability between measures was not statistically significant as the ICC value of 0.04 was not significantly different from $0(\mathrm{~F}(1,57)=3.37, p=.07)$. With regard to weighted sedentary behaviour/sitting time, the ICC value of -0.01 was also not significantly different from $0(\mathrm{~F}(1,57)=0.16, p=.69)$.

Although both devices were worn continuously for a 3-day period, it was not possible to compare sleep data recorded by both devices. There are no established guidelines or validation studies for the use of Acticals or activPALs to measure sleep in preschoolers, therefore it was not possible to meaningfully assess and compare accelerometer data recorded during sleep periods. 
Table 9. Descriptive characteristics of participants who wore Actical and activPAL accelerometers in the Canadian SUNRISE pilot study.

\begin{tabular}{|c|c|}
\hline Variable & Full sample $(\mathrm{n}=31)^{\mathrm{a}}$ \\
\hline Age (years) & $4.5 \pm 0.3^{b}$ \\
\hline \multicolumn{2}{|l|}{ Sex } \\
\hline Male & $61 \%(n=19)$ \\
\hline Female & $39 \%(n=12)$ \\
\hline \multicolumn{2}{|l|}{ Mean daily wear time (mins) } \\
\hline Actical & $803 \pm 70.2$ \\
\hline $\operatorname{activPAL}$ & $836 \pm 132.5$ \\
\hline \multicolumn{2}{|l|}{ Mean daily steps } \\
\hline Actical & $11340.4 \pm 4497.0$ \\
\hline $\operatorname{activPAL}$ & $10910.9 \pm 3664.4$ \\
\hline \multicolumn{2}{|l|}{$\begin{array}{l}\text { Mean daily sedentary/sitting time } \\
\text { (mins) }\end{array}$} \\
\hline $\begin{array}{r}\text { Actical } \\
\text { activPAL }\end{array}$ & $\begin{array}{c}439.8 \pm 87.8 \\
466.5 \pm 130.4\end{array}$ \\
\hline
\end{tabular}

${ }^{a}$ Mean and standard deviation values were presented for continuous variables, and percentage and frequency for categorical variables.

$\mathrm{b}_{ \pm}$Standard deviation

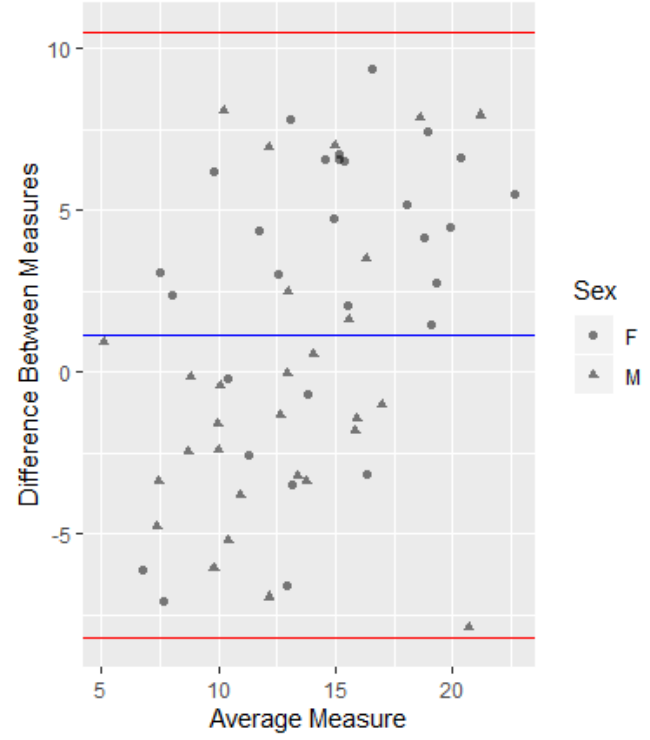

Figure 4. Bland-Altman plot of differences between daily step counts weighted by wear time measured by activPAL relative to Actical accelerometers $(n=58)$. 


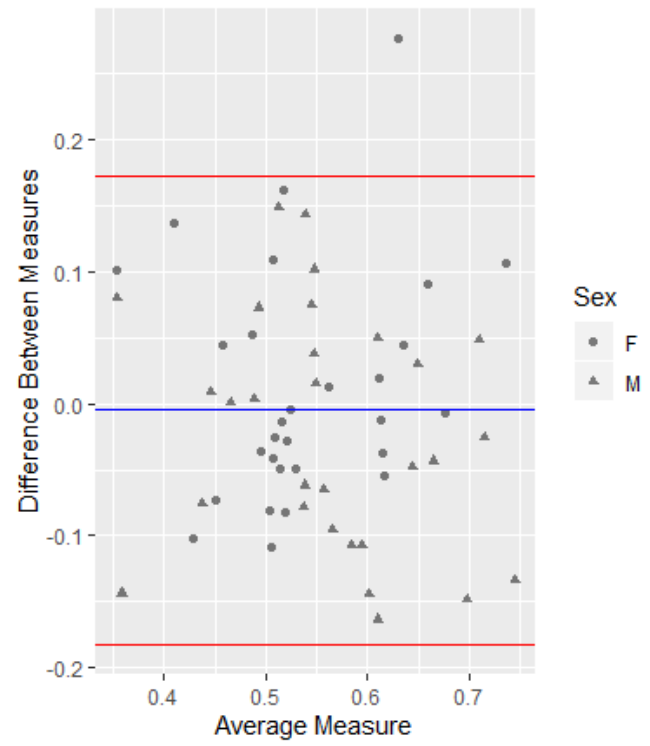

Figure 5. Bland-Altman plot of differences between sedentary time/sitting time weighted by wear time measured by activPAL relative to Actical accelerometers $(\mathrm{n}=$ 58). 


\section{Discussion}

\section{Feasibility of the SUNRISE Study (Objective 1)}

The first objective of this project was to assess the feasibility of the SUNRISE study measurement instruments and protocols. Overall, the Canadian SUNRISE pilot study revealed mixed findings regarding the feasibility of the SUNRISE study.

\section{Feasibility of Recruitment}

In terms of achieving the target sample size of 100 participants (50 urban and 50 rural), low enrollment rates at both childcare centres (10.3\%) and schools (17.7\%) limited the study's ability to reach this target in a timely or efficient manner. Although a sample size of 106 participants was eventually reached, doing so required research staff to contact all of the 169 schools in the OCDSB and OCSB. Response rates in the current study were low compared to other similar physical activity studies conducted within schools in Ottawa. For example, a multi-site study on active transportation and independent mobility that was conducted in Ottawa schools had a response rate of $60.6 \% .{ }^{194}$ Additionally, in the Canadian ISCOLE study, accelerometry and questionnaire data were collected from over 500 school-aged children attending Ottawa schools with a response rate of $50 \% .{ }^{195}$ In terms of childcare centres, a randomized controlled study of a physical activity intervention among preschoolers attending childcare centres in Ottawa had a $66 \%$ response rate. ${ }^{196}$ The lower response rates in the SUNRISE study may be partly explained by a lack perceived attractiveness of movement behaviour research among parents. Relative to research that is grounded in established topics such as obesity, active transportation, 
and childcare-based physical activity interventions, a surveillance study of movement behaviours may not carry a similar degree of perceived importance among parents.

The target sample of 50 urban children was met $(n=83)$, however the target of 50 rural participants was not met $(n=23)$ due in part to the study's research ethics rejection from the UCDSB. The current study's research ethics application was rejected due to a perceived lack of direct benefits that the study would provide the UCDSB. This requirement by the UCDSB was unforeseen and recruitment in the UCDSB was intended to fulfill the rural sample. Instead, the rural sample consisted of participants from 3 rural childcare centres and 2 rural OCDSB schools. Study recruitment outside of the summer period was entirely dependent on being able to recruit within schools. Due to this dependency on schools, a failure to gain research ethics approval from the school boards could severely impair recruitment for the main Canadian SUNRISE study if it were to occur solely in Ottawa. Given that the target sample size of the main SUNRISE study is 500-1000 (with an even urban and rural split) participants per country, it is unlikely that the City of Ottawa alone would be a feasible location to conduct the main Canadian study. Instead of recruiting solely in the Ottawa area, it would be beneficial to spread recruitment across multiple sites throughout Canada. Moreover, the Canadian pilot study sample was likely nonrepresentative and was skewed towards Caucasian participants with post-secondary household educational attainment. It is recommended that the main SUNRISE study employ a methodology that closely resembles a random sampling approach to maximize representativeness, although this may prove to be an issue given the difficulties experienced in Ottawa with recruitment. 
From conversations with the principals of schools, there were several instances of principals expressing interest in participating in the SUNRISE study but declining to participate due to their school's recent participation in another study. The City of Ottawa has two public universities and two public colleges; all potentially competing to conduct research studies within the Ottawa school boards. The oversaturation of research demands among the schools in Ottawa is a potential explanation for the difficulties recruiting schools to participate in the Canadian SUNRISE study. Results from a qualitative study on why people participate in epidemiological research indicated that individuals are more likely to participate in research that they believe provides a broad public benefit or in research that is focused on an illness that they, their family, or their friends have been impacted by. ${ }^{197}$ A Canadian study also reported that participation was positively influenced by the potential to provide future health benefits to themselves or society and receiving a copy of study results. ${ }^{198}$ Provided that movement behaviours are not established pathologies, it is possible that the parents of eligible children at participating sites did not view movement behaviours as a study topic that was relevant to them or their children. In this regard, research has found that many parents believe that their preschool-aged children are sufficiently active. ${ }^{199}$ Therefore, parents approached for the current study potentially may not have perceived research on physical activity and related movement behaviours as a priority. Additionally, in the SUNRISE study documents it was not explicitly stated that parents could receive a copy of their child's results, which may have deterred some parents from consenting to their child's participation in the study. This speculation is further supported by comments 
in parent/guardian feedback documents in which several parents stated their interest in seeing their child's study results.

\section{Feasibility of Accelerometry}

In general, participants adhered to the Actical wear protocol and enjoyed wearing the device. One reoccurring complaint from parents/guardians was that the belts used to wear Acticals were too large, uncomfortable for their child, or falling off their child (15\%). Size "small" belts were used in this study; however the use of smaller belts would have likely mitigated reported wear issues. Well-established physical activity intensity cut-points facilitated data reduction and there were no reported negative physical or behavioural reactions to wearing Acticals. Acticals have been validated for use among preschool-aged children, ${ }^{167,175}$ and they have been used in a large-national study of the physical activity of Canadian preschool-aged children. ${ }^{127}$ Based on this evidence, Acticals appear to be a feasible instrument for assessing physical activity in the SUNRISE study.

One issue with activPAL accelerometers was the lack of consistency among validation studies of physical activity intensity determined by activPALs among preschool-aged children. ${ }^{176,179,182}$ The new WHO movement guidelines for children under 5 years of age provide physical activity recommendations based on total physical activity and MVPA. ${ }^{2}$ Therefore, it is critical that the devices used to assess adherence to this component of the guidelines be able to correctly categorize physical activity intensities. Additionally, the inconvenience of applying activPALs to participant's thighs when they were wearing pants/leggings added time and a degree of invasiveness to the data collection process. Of 33 participants that wore activPALs 
in the Canadian SUNRISE pilot study, there were 4 (12\%) reports of skin irritation associated with the wear of activPALs. Rashes/irritation were reported to occur on the area the children's thighs where the waterproofed activPALs were in direct contact with the skin. Following these reports of rashes/irritation, activPALs were removed from the Canadian SUNRISE pilot study and appropriate follow-up was carried out with affected parents/guardians and relevant research ethics boards. Consistent with the reports of irritation in the current study, other studies have also reported similar issues associated with the continuous wear of activPALs. ${ }^{176,200}$ In one of the studies, $39 \%$ of the adolescent participants reported skin irritation associated with wearing activPALs and 55\% reported that they would rather not wear the device again. ${ }^{200}$ In another study of 4-6 year-old children, $38 \%$ of parents reported that their children had skin irritation due to wearing activPALs for consecutive days. ${ }^{176}$ Based on these findings, activPALs are not feasible accelerometers to be included in the SUNRISE study.

In consideration of the 3-day accelerometer protocol, the short data collection period limited the amount of days available for analysis. Two valid days was the maximum amount available for analysis in this sample, and participants with only 1 valid day were not included in analyses. Findings from a study by Penpraze and colleagues $^{201}$ indicated that a 7-day protocol maximizes the reliability of physical activity estimates in 5 year-old children, but also suggest that a minimum of a 3-day protocol may be sufficient. Another study of physical activity measurement in children 1-5 years-old found that any 2 valid weekdays or weekend days of accelerometer data were sufficient for measuring physical activity. ${ }^{202}$ Consistent with 
these results, findings from Hislop and colleagues suggest that 3 days, regardless of the inclusion of a weekend day, provided sufficient reliability for characterizing physical activity in 3-4 year-old children. ${ }^{203}$ Although it appears that the current 3day protocol (no weekends included) of the SUNRISE study is feasible, in order to maximize the number of participants included in analyses, the study protocol would likely benefit from a longer accelerometer protocol. For example, a nationallyrepresentative study of movement behaviours among preschool-aged children used a 7-day protocol with the inclusion of participants with 3 or more valid days. ${ }^{127}$ Expanding the length of the protocol would provide more allowance for non-wear, and could improve the reliability of physical activity estimates.

\section{Feasibility of Motor Skills Testing}

Overall, ASQ testing was simple to administer, not excessively time consuming, and was well tolerated by participants in this study. Additionally, the ASQ has been employed internationally in a range of contexts without a significant attenuation in performance. ${ }^{204}$ Thus, the ASQ appears to be a good test for an international surveillance study. However, as noted previously, the ASQ was designed to be completed by a parent or a trained professional who is familiar with the child. ${ }^{204}$ In this study, researchers who were unfamiliar with the participants were administering the tests, therefore some questions arise as to the validity of the test when administered in this manner. At all study sites it was not possible to score the gross motor item related to climbing a ladder and sliding down a slide due to a lack of access to readily available slides, although the scoring of the ASQ allows for adjusted scoring to account for missing items. ${ }^{205}$ It is advisable that in the main SUNRISE 
study, the motor skills testing protocol should either be modified to have the ASQ scored by the parents/guardians of participants, which might present its own feasibility issues, or be changed for a different battery of validated motor tests.

Alternative motor development tests include the Bayley Scales of Infant Development (BSID II), however the BSID II also comes with its own limitations in that it is costly, time consuming, and requires a trained professional to administer the test. ${ }^{206}$

\section{Feasibility of Executive Function Testing}

In general, participants disliked the EYT games included in the study protocol. Contrary to how the games are described by their creators as being short $(<5$ mins) and engaging, ${ }^{188}$ many participants found the games to be excessively long and unengaging. Given the ubiquitous nature of screens in society and the high prevalence of screen time among children, ${ }^{95}$ it is surprising that many children found screen time in the form of the EYT games uninspiring. The scores of some participants may have been inaccurate due to mid-game distractions that caused the child to lose focus from the game. Issues that children had with remaining engaged with games and completing them properly suggest that potential alternative measures of executive function should be investigated. Although the EYT protocol in the SUNRISE study still appears to feasible, an alternative battery of games such as the NIH Toolbox (National Institutes of Health, Bethesda, MD, United States) should be explored as a potentially more suitable alternative.

\section{Feasibility of Parent/Guardian Questionnaire}

Parents reported several issues related to the wording of items in the parent/guardian questionnaire. Questions beginning with "In a 24 hour period in the 
past week" caused confusion among parents and it is recommended that the question be reworded to be clearer. Additionally, it is recommended that the item related to the sex of the "main caregiver" be changed to reflect the fact that in the Canadian context many parents share equal caregiving responsibility for their children.

Many parents also reported that their estimations of their child's physical activity were inaccurate because they were not fully aware of their child's activity while at childcare centres/schools. Despite this, results from a study comparing parent reported and accelerometer measured physical activity among children under 6 years of age found weak to moderate correlations between the two measures, with the highest correlations between parent reported unstructured physical activity inside daycare/school and accelerometer-measured total physical activity. ${ }^{207}$ Therefore, it is possible that as a result of the structured nature of childcare centres/schools, parent reported physical activity in this study had higher accuracy than was initially perceived by parents.

In relation to questions on physical activity, a number of parents commented about perceived seasonal variation in their child's physical activity. Several studies have found that these concerns are well-founded. A systematic review found evidence that physical activity varies seasonally, and poor weather was identified as a barrier to physical activity. ${ }^{208}$ In a study of preschool children in Edmonton, Canada, there were significant seasonal differences in total physical activity, weekday physical activity, and weekend physical activity, with children less likely to be physically active in the winter. ${ }^{209}$ These findings were in contrast to a Finnish study of 3 year-old children where seasonality was found to only minimally influence children's physical 
activity. ${ }^{210}$ Given that Canada's climate varies considerably throughout the year, ${ }^{211}$ seasonality likely plays a role in influencing a child's physical activity. In the Canadian SUNRISE pilot study, data were collected from participants across all seasons. Therefore, it is advisable that in the main SUNRISE study, the season of the data collection period be taken into account.

There were not any issues related to the screen time or sleep items in the parent/guardian questionnaire. Although, as both measures were parent-reported, social desirability bias may have led to underestimation of their child's screen time, while erroneous recall may have resulted in overestimations. ${ }^{212}$ The objective assessment of sleep duration is relatively well established, ${ }^{213}$ and the main SUNRISE study would likely benefit from using actigraphy to assess adherence to sleep guidelines in order to reduce the risk of bias or reporting error. Objective means for assessing the screen time of preschool-aged children are still underdeveloped and especially in the context of a large international surveillance study, subjective parent reports are likely the most feasible protocol for the main SUNRISE study.

\section{4-Hour Movement Behaviour Adherence (Objective 2)}

Findings from the Canadian SUNRISE pilot study indicate that few participants were adhering to the WHO's movement guidelines for the early years. ${ }^{2}$ In this study, $19.8 \%$ of participants were adhering to all 3 movement behaviour recommendations, $48.8 \%$ were adhering to two recommendations, $25.6 \%$ were adhering to one recommendation, and $5.8 \%$ were not adhering to any recommendations. More specifically, $76.7 \%$ of participants were adhering to sleep recommendations, $55.8 \%$ to physical activity recommendations, and $50 \%$ to 
sedentary behaviour recommendations. There were also no significant differences in the number of recommendations met by sex, weight category, or urban/rural location.

These results were consistent with those of a nationally-representative Canadian study, ${ }^{127}$ which found similar proportions in terms of the number of movement behaviours recommendations met $(12.7 \%, 48.0 \%, 36.0 \%$, and $3.3 \%$ of $3-4$ year-old children were meeting all, two, one, and none of the recommendations from the Canadian movement guidelines for the early years, respectively). ${ }^{57}$ Similar to the Canadian SUNRISE pilot study, the study used Acticals and the same activity count cut-points to objectively assess physical activity adherence and parent-reports to assess screen time and sleep adherence. The WHO movement behaviour guidelines for the early years ${ }^{2}$ were based on the Canadian guidelines ${ }^{3}$ and Australian guidelines $^{4}$ and composed of essentially identical recommendations for movement behaviours.

Regarding individual movement recommendations, findings were only somewhat consistent with previous studies. In the current study, most participants were meeting sleep recommendations (76.7\%), which was relatively similar to findings from several Canadian and Australian studies of movement behaviours in the early years (82-88\%). ${ }^{127,151-153}$ However, although physical activity adherence (55.8\%) approximated results by Chaput and colleagues $(61.8 \%),{ }^{127}$ substantially less were adhering to recommendations in the Canadian SUNRISE pilot compared to other Canadian and Australian studies (93-99\%). ${ }^{151,153}$ In a study by Carson and colleagues, the opposite trend was found, where only $19 \%$ of 3-4 year-old participants were meeting physical activity recommendations. These findings may be partially 
explained by the use of similar devices (Acticals) and cut-points by Canadian SUNRISE pilot study and the study by Chaput and colleagues. ${ }^{127}$ The other aforementioned studies utilized ActiGraph accelerometers (ActiGraph, Pensacola, FL, United States), which have different associated cut-points based on the devicespecific activity counts. As well, except for the study by Chaput and colleagues, ${ }^{127}$ the other previous studies did not include representative samples, which may have contributed to the observed differences in proportions adhering to physical activity recommendations. Lastly, regarding sedentary behaviour/screen time adherence, the results from the Canadian SUNRISE pilot study (50\%) was similar to findings from a Canadian study involving 3 year-old children from Edmonton, where $50.5 \%$ of participants were found to be meeting screen time recommendations. ${ }^{152}$ This is in contrast to other studies where significantly fewer participants were meeting screen time recommendations (5-24\%). ${ }^{104,127,151,153}$ Overall, it appears that most children are meeting sleep recommendations, however findings are mixed in terms of the proportions meeting physical activity and sedentary behaviour/screen time recommendations.

Differences in proportions adhering to movement recommendations between studies are potentially attributable to the more stringent criteria used in the Canadian SUNRISE pilot study to determine adherence to sedentary behaviour and sleep recommendations. In other studies, ${ }^{127,151-153}$ adherence to sedentary behaviour/screen time recommendations was defined as no more than $1 \mathrm{hr}$ of daily screen time, and sleep recommendations were defined as 10-13 hrs of sleep per day. However, adherence to sedentary behaviour recommendations in Canadian SUNRISE pilot 
study was defined as no more than $1 \mathrm{hr}$ of daily screen time and not restrained while seated for more than $1 \mathrm{hr}$ at a time. In addition, sleep recommendation adherence in this study was defined as 10-13 hrs of sleep per day with consistent wake and sleep times. These additional movement behaviour recommendation caveats likely decreased the proportion of children meeting sedentary behaviour and sleep recommendations compared to other studies. In the case of sleep adherence, using less stringent criteria likely would have increased adherence and brought results closer to findings from other studies, however with regard to screen time, less stringent definitions would have exacerbated the differences in findings (i.e., increased already higher adherence proportions relative to other studies). Additionally, the current study's sample was not representative and was biased towards higher household educational attainment and Caucasian ethnicity, which may have contributed to differences in findings.

The lack of differences between sexes and weight categories are consistent with movement behaviour findings from Chaput and colleagues who reported similar findings among a nationally-representative sample of 3-4 year-olds. ${ }^{127}$ These findings suggest that, potentially, sex difference in adherence to movement guidelines do not appear until after the early years. In the current study and the study by Chaput and colleagues, ${ }^{127}$ most participants were normal weight or at risk of overweight, therefore there may have been inadequate statistical power to detect differences between significantly different weight categories (e.g., normal weight compared to obese). No studies have previously assessed urban/rural differences in movement guideline adherence in a preschool-aged sample. One study of Mozambican children 
found differences between urban (3.6\%) and rural (17.7\%) children meeting all 3

movement recommendations. ${ }^{154}$ However, that study involved an older population (911 year-olds) and the differences between urban and rural Mozambique are likely quite significant. In contrast, the differences between urban and rural Ottawa are less distinct, which may have attenuated potential differences in proportions meeting guidelines.

In short, the results from the Canadian SUNRISE pilot study indicate that half of participants were not meeting sedentary behaviour guidelines and nearly half were not meeting physical activity recommendations. In contrast, a relatively high proportion of participants were meeting sleep recommendations. From what is known regarding the potential health consequences of unfavourable levels of movement behaviours in the early years, ${ }^{107}$ the findings in this study support the need for interventions to increase physical activity and decrease sedentary behaviour levels among young Canadian children. The full 24-hour period matters; and although many children were meeting sleep recommendations, associated benefits of meeting recommendations for this behaviour may be attenuated by unhealthy physical activity and sedentary behaviours.

\section{International Screen Time Comparison (Objective 3)}

Based on the SUNRISE pilot data from Brazil, Canada, China, Papua New Guinea, South Africa, Sweden, United States, and Zimbabwe, 3-4 year-old participants averaged roughly 100 mins of screen time per day and $51 \%$ of participants were meeting screen time recommendations. Brazilian participants had the lowest screen time adherence (20\%) and participants from China and Papua New 
Guinea had the highest adherence (66\%). There was significant variation in adherence proportions between countries based on non-parametric tests with statistically significant differences between Brazil and Canada, Brazil and China, Brazil and Papua New Guinea, China and Sweden, and Papua New Guinea and Sweden. In addition, logistic regression models controlling for the same descriptives indicated that participants from very high HDI countries had a 0.6 odds of meeting screen time recommendations relative to those from high HDI countries. Even more strikingly, participants from low to medium HDI countries had 2.7 times the odds of meeting screen time recommendations relative to those from very high HDI countries. Similarly, statistically significant differences in screen time between very high HDI countries and medium to low HDI countries were found in non-parametric tests, with higher screen time in very high HDI countries. There were also significant differences between the highest household educational attainment categories primary school/some high school and high school based on non-parametric tests. Although, this relationship was not linear with the primary school/some high school group having the highest adherence (66\%), which was then followed by the post-secondary group (52.6\%), and finally by the high school group (42.7\%). There were also differences in daily screen time between highest educational attainment groups. There were no significant differences in screen time adherence proportions between sexes, weight categories, or urban/rural location.

The proportion of participants adhering to screen time recommendations was higher among these pilot samples than findings from other Canadian and Australian studies, which reported that 5-24\% were meeting recommendations. ${ }^{104,127,151,153}$ 
However, findings were consistent with one Canadian study of movement behaviours among 3 year-old children (50.5\%). ${ }^{152}$ The findings were roughly consistent with results from the ISCOLE study, where $45.8 \%$ of participants (9-11 years-old) were adhering to screen time recommendations (no more than $2 \mathrm{hrs}$ recreational screen time per day). ${ }^{103}$ The ISCOLE study had a sample composition similar to this current study and included samples from Australia, Brazil, Canada, China, Colombia, Finland, India, Kenya, Portugal, South Africa, the United Kingdom, and the United States. However, the ISCOLE study included an older participant group (9-11 yearolds) with a different screen time threshold and the samples were larger and more representative than those in the current study. Interestingly, the SUNRISE pilot study screen time results for China were in contrast to those from the Global Matrix 3.0 of Physical Activity Report Cards for Children and Youth. ${ }^{214}$ In the Global Matrix 3.0, China was assigned a grade of "F", which was informed by a nationallyrepresentative study indicating that only $7.1 \%$ of $9-17$ year-olds are meeting screen time recommendations of no more than $2 \mathrm{hrs}$ of daily screen time. ${ }^{215}$ These findings from the Global Matrix 3.0 were in contrast to the results from the SUNRISE pilot study results where $66 \%$ of $3-4$ year-old participants were adhering to screen time recommendations. These results may partially be explained by the significant variation in the size of each country's sample, the non-representativeness of samples included in the pilot studies, differences in measurement procedures, and differences in ages.

Based on results from the Global Matrix 3.0, which involved the participation of 49 countries from a range of HDI backgrounds, the mean letter grade for sedentary 
behaviour was " $\mathrm{D}+$ ". ${ }^{214} \mathrm{~A}$ " $\mathrm{D}+$ " generally corresponds to $34-39 \%$ of children meeting sedentary behaviour recommendations of no more than $2 \mathrm{hrs}$ of daily screen time for school-aged children and youth. ${ }^{214}$ Breaking this aggregated grade down, medium to low HDI countries had a mean grade of " $\mathrm{C}+$ ", high HDI countries had a mean grade of "D", and very high HDI countries had a mean grade of "D+". These results are similar to the international screen time results of the current study where a similar negative linear gradient was observed between screen time and HDI levels (i.e., decreasing screen time recommendation adherence with increasing HDI values). Despite these trends, sedentary behaviours in the Global Matrix 3.0 were not significantly related to HDI, though this analysis may have been under-powered. Data used from the Global Matrix 3.0 were from an older age group (5-17 year-olds) than the current study and were collected using differing methodologies and benchmarks. ${ }^{214}$

Although there were significant differences between highest household educational groups in terms of adherence to screen time guidelines and total daily screen time, the specific nature of these relationships is not clear. One study of Chinese preschoolers found that higher daily screen time was associated with lower maternal education. ${ }^{216}$ Another study of youth screen time, which involved a metaanalysis of various international data from the ICAD, similarly found that participants with higher maternal education were more likely to have less than $2 \mathrm{hrs}$ of daily screen time ${ }^{88}$ These findings are in contrast to observations in the current study where the primary school/some high school category had the highest screen time adherence. With regard to sex, weight category, and urban/rural differences in daily 
screen time and adherence to screen time recommendations, the current study did not find any statistically significant differences between groups. These findings are in contrast with previous studies where boys were found to have higher screen time and poorer adherence to screen time guidelines than girls, ${ }^{88,103}$ children with a poorer weight status were more likely to exceed screen time recommendations than those with a normal weight status, ${ }^{88,103}$ and children in rural locations were more likely to meet screen time recommendations than children from urban locations. ${ }^{154,217,218}$ Potential explanations for these contrasting results include a younger study sample in the current study, relatively small and variable sample sizes among SUNRISE pilot countries, and the potential loss of cultural sensitivity through the recoding of highest household educational attainment levels.

Overall, nearly half of the preschool-aged participants from the SUNRISE pilot studies were not meeting screen time recommendations, which provides further evidence that many young children do not have healthy levels of sedentary screen time behaviour. Participants from very high HDI countries were found to have significantly lower adherence to screen time recommendations than participants from medium to low HDI countries. These trends are of concern given that many medium to low HDI countries, where children are relatively succeeding in terms of sedentary behaviours, are undergoing rapid economic transitions and becoming more like the high and very high HDI countries, ${ }^{219}$ where children have less favourable sedentary screen time behaviours. Despite these findings, there remains the need for nationallyrepresentative surveillance studies investigating screen time in the early years to 
further clarify estimates of screen time adherence and its relationships with various correlates.

\section{Comparison of Actical and activPAL Physical Activity, Sedentary}

\section{Behaviour, and Sleep Data (Objective 4)}

As part of the Canadian SUNRISE pilot study, comparative analyses of Actical and activPAL step counts and sedentary/sitting time were performed. Based on 58 participant-days of comparison, it was found that Actical and activPAL step counts and sedentary/sitting time weighted by wear time have poor agreement. Although paired t-tests did not reveal any statistically significant differences between means of the measures, based on the computed ICC values there was poor reliability between Actical and activPAL measures of steps and sedentary/sitting time. BlandAltman plots that were generated did not reveal any significant measurement biases between Actical and activPAL outputs. Although there was visual evidence for a tendency of Acticals to measure less steps than activPALs when average steps per min of wear time was lower, and the opposite trend was observed for higher steps per min of wear time. These findings suggest that variation between Actical and activPAL measures were not biased in a consistent manner and were instead spread randomly, which is difficult to correct for. Additionally, it was not possible to compare sleep time due to a lack of validated algorithms for extracting sleep from Actical and activPAL data.

The step count findings from the Canadian SUNRISE pilot study were in contrast to those from a study by Aminian and colleagues, where it was found that Acticals recorded $33 \%$ more steps than activPALs among 5-11 year-old children. ${ }^{220}$ 
Another study of adults in a laboratory setting found a correlation of 0.66 between Actical and activPAL step counts. ${ }^{221}$ In terms of sedentary behaviour, a study involving preschool aged children found that although no systematic biases were apparent in Bland-Altman plots, sedentary behaviour as measured by Acticals and activPALs indicated moderate agreement and there was large random bias between measures. ${ }^{222}$ These results were somewhat consistent with the current study where no systematic biases were found between Actical and activPAL sedentary/sitting time. Although, their study found moderate agreement between measured sedentary times, which was in contrast to results from the current study.

A potential explanation for these observed differences between this study and previous studies was the methodology employed in the Canadian SUNRISE study. The accelerometer protocol in this study was not designed to maximize the comparability of Actical and activPAL measures. Daily wear time varied per participant and often varied per device as devices would fall off or were removed during waking hours. To correct for this variation, daily step counts and sedentary/sitting time were weighted by wear time for that day. This crude correction may have resulted in a loss of sensitivity compared to a protocol ensuring equal and time-matched wear time and direct comparisons of measures had been employed. Moreover, comparisons were performed at the day level in this study, whereas comparisons at the epoch level may have yielded differing results. In the study of sedentary behaviour comparisons by Van Cauwenberghe and colleagues, comparisons were performed on every $15 \mathrm{sec}$ epoch for each full day of data. ${ }^{222} \mathrm{In}$ addition, Actical sedentary time was based on established activity count cut-points, 
whereas activPAL sitting time was determined with the positional data indicating a sitting or lying posture. Therefore, these differing criteria may have biased the types of activities being classified as sedentary behaviour by both devices. Lastly, the contrasting findings from the paired t-tests and ICC values are potentially explained by the random variation in step and sedentary/sitting time data. Paired t-tests are used to test differences between the means of two samples. ${ }^{223}$ However, the random variation between Actical and activPAL measures may have resulted in equal variation above and below the mean, which equated to mean differences that were relatively small. In contrast, the ICC is defined as the ratio of between-pair variance to the total variance. ${ }^{224}$ Therefore, the ICC was not influenced by random variation of the Actical and activPAL measures.

In summary, it appears that the use of Actical versus activPAL devices is not inconsequential, and the choice of device may have implications for step count and sedentary behaviour results. Although results from the current study should be interpreted cautiously, supporting evidence from other studies suggest that Acticals and activPALs yield different step and sedentary behaviour data. Further research is needed to develop activPAL physical activity intensity cutoffs to compare the classification of physical activity intensities between devices. Additionally, more comparative studies among preschool-aged populations are needed due to differences between the movement patterns of young children relative to older children and adults. Finally, sleep algorithms need to be developed in order to assess and compare sleep data recorded by Actical and activPAL devices. 


\section{Strengths and Limitations}

There were several strengths of the Canadian SUNRISE pilot study and the international comparison of screen time data from the SUNRISE pilot study samples. In this study, physical activity recommendation adherence was objectively assessed by accelerometry. As previously described, subjective means of assessing physical activity, such as parent reports, have a number of limitations for 4 year-olds, ${ }^{52-55}$ and therefore the use of accelerometers in this study provide direct insight into the physical activity of participants. The intended urban/rural balance of the study samples allows for the analysis of an important demographic independent variable that has been understudied, especially in the early years. The international comparisons of screen time data included 8 countries from a diverse range of HDI backgrounds, which allowed for analyses based on HDI groupings.

The Canadian SUNRISE pilot study and the international comparison of screen time data also had several limitations. First, the pilot study samples were not representative and varied in size, which imposes limitations on the generalizability of the results. In addition, in the Canadian SUNRISE pilot study the maximum number of valid days of accelerometer wear was limited to 2 days. Regarding accelerometry, objective methods are not without their own limitations in that they are unable to capture the full extent of bodily movements, they may accidentally capture nonphysical activity, they may be overly sensitive to small errors in calibration methods and cut-points used to categorize physical activity, and they provide no information on context (e.g., indoors versus outdoors, alone or with a group, at home or at school).${ }^{54}$ In the Canadian study, study documents were only available in English, 
which excluded Francophones and participants from non-English speaking immigrant backgrounds. Additionally, the parent-report measures used to assess screen time and sleep recommendation adherence may have been biased and objective measures would have been ideal. The comparison of Actical and activPAL results are likely not generalizable due to the methodology used in the Canadian SUNRISE pilot study.

\section{Future Directions}

The findings from the SUNRISE pilot studies are intended to inform the design of the main SUNRISE surveillance study. Changes will be made to the main study's protocol and measurement instruments based on issues identified in the Canadian pilot study and the pilot studies carried out in other countries. The main SUNRISE study will likely include the participation of over 30 countries and will be the first study to provide global estimates of levels of movement behaviours in the early years. Additionally, the main SUNRISE study will facilitate capacity building in low- and medium-income countries and create a global network of researchers investigating movement behaviours among young children. Future research should be aimed at developing sleep algorithms for objectively assessing sleep with Actical and activPAL accelerometers. Additionally, future work should target the development of activity count cut-points for preschool-aged children using activPAL accelerometers, and new protocols should be investigated for minimizing irritation associated with wearing the devices. 


\section{Conclusion}

In summary, results from the Canadian SUNRISE pilot study revealed mixed findings in terms of the feasibility of the study protocol in a Canadian context and helped to identify areas for improvement. Most sampled children from the Canadian SUNRISE pilot study were not adhering to the WHO's Guidelines on Physical Activity, Sedentary Behaviour, and Sleep for Children Under 5 Years. ${ }^{2}$ Many participants were meeting sleep recommendations, however only approximately half of participants were meeting recommendations for physical activity and sedentary behaviour. International screen time data from the Brazil, Canada, China, Papua New Guinea, South Africa, Sweden, United States, and Zimbabwe SUNRISE pilot studies suggests that nearly half of participants were not meeting screen time recommendations of no more than $1 \mathrm{hr}$ of screen time per day. The proportion of these participants adhering to screen time recommendations was also found to differ between highest household education categories and HDI groups. Lastly, there was poor reliability between steps and sedentary/sitting time measured by Acticals and activPALs though there were no consistent biases in these measures. Overall, the findings from this project will help to inform the main SUNRISE surveillance study, and contribute to better understanding movement behaviours in the early years. 


\title{
APPENDICES
}

\section{APPENDIX 1. Most-Recent Research Ethics Approvals}

\section{Carleton}

\author{
Office of Research Ethics \\ 503 Robertson Hall | 1125 Colonel By Drive \\ Ottawa, Ontario K1S 5B6 \\ 613-520-2600 Ext: 4085 \\ ethics@carleton.ca
}

\section{CERTIFICATION OF INSTITUTIONAL ETHICS CLEARANCE}

This document is an administrative clearance corresponding to the amendment to protocol \#18/19X cleared by the CHEO Research Ethics Board on March 4, 2019. CUREB-B is constituted and operates in compliance with the Tri-Council Policy Statement: Ethical Conduct for Research Involving Humans (TCPS2).

Ethics Clearance ID: Project \# 109584

Principal Investigator: Mr. Evan Tumer

Co-Investigator(s) (If applicable): Mr. Evan Turner (Primary Investigator) Paul Villeneuve (Research Supervisor)

Project Title: SUNRISE Pilot Study: Canadian Surveillance study of Movement Behaviours in the Early Years [Evan Turner]

\section{Funding Source:}

Effective: August 02, 2019

Expires: August 31, 2020.

Upon reasonable request, it is the policy of CUREB, for cleared protocols, to release the name of the PI, the title of the project, and the date of clearance and any renewal(s).

During the course of the study, if you encounter an adverse event, material incidental finding, protocol deviation or other unanticipated problem, you must complete and submit a Report of Adverse Events and Unanticipated Problems Form, found here: https://carleton.ca/researchethics/forms-and-templates/

Please email the Research Compliance Coordinators at ethics@carleton.ca if you have any questions. 
From: nanderson@cheo.on.ca [mailto:nanderson@cheo.on.ca]

Sent: September-24-19 8:08 AM

To: Tremblay, Mark

Cc: Carson, Stephanie; Anderson, Natalie

Subject: REB Protocol No 18/19X - Approval of Minor Modification

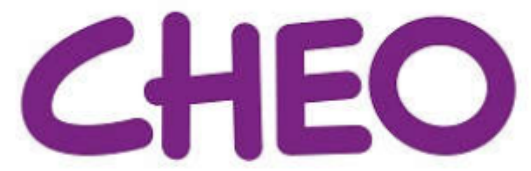

\section{CHEO Research Ethics Board Approval - Minor Modification}

REB Protocol No: 18/19X

ROMEO File No: 20180098

Principal Investigator: Dr. Mark Tremblay

Project Title: CHEOREB\# 18/19X - SUNRISE Pilot Study International Surveillance study of Movement

Behaviours in the Early Years

Protocol Status: Active

Date Modifications Approved: September 23, 2019

Documents Reviewed \& Approved:

Document Name Comments Version Date

\begin{tabular}{l|l|l|}
\hline Protocol & Protocol & $2019 / 09 / 18$ \\
\hline
\end{tabular}

The CHEO REB has reviewed and granted approval of the study documents listed above; which is to be conducted under the auspices of CHEO and/or CHEO Research Institute by the investigator noted above. The minor modification was reviewed in the delegated stream and approved by the Chair, and will be ratified by the full Board at its subsequent meeting.

Approval is granted with the understanding that the investigator agrees to comply with the following requirements: 
Stephanie Carson

CHEO Research Institute

401 Smyth Road

Ottawa, Ontario

K1H $8 \mathrm{~L} 1$

\section{Re: SUNRISE Pilot Study}

Dear Ms. Carson,

The Ottawa-Carleton Research and Evaluation Advisory Committee has reviewed the revisions to your application and is granting you formal approval to conduct your study in the 2018-2019 academic year. This approval is for the Ottawa Catholic School Board and the Ottawa-Carleton District School Board. However, please note that final approval to participate in the study must come from the individual principal.

At the time in which your research team is in our schools, the committee asks that police record checks and a copy of this letter of approval be shown to the school staff being approached to participate in your study. We thank you for approaching the Ottawa-Carleton area school boards as a venue for your study and we look forward to receiving a copy of your results.

Sincerely,

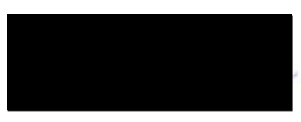

Lauren Figueredo, Ph.D

Research Officer, Leading \& Learning

Ottawa Catholic School Board

613-224-4455 ext. 2341

lauren.figueredo@ocsb.ca

On behalf of the Ottawa-Carleton Research and Evaluation Advisory Committee 


\section{APPENDIX 2. Parent/Guardian Package Documents}
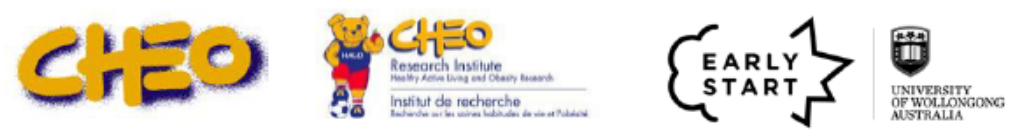

COVER LETTER

INTERNATIONAL SURVEILLANCE STUDY OF 24-HOUR MOVEMENT BEHAVIOURS IN THE EARLY YEARS: The SUNRISE Study

Dear Parent/Guardian,

Researchers from the Children's Hospital of Eastern Ontario (CHEO) Research Institute, in collaboration with the University of Wollongong, in Australia, are conducting a multi-country research study to examine the levels of physical activity, sedentary behaviour, and sleep among approximately 4-year-old children attending elementary schools/childcare centres. The elementary school/child care centre your child attends agreed to give you this package. We are hoping you and your child will participate in this important research study that will support the health and wellbeing of young children.

Please read the attached information letter for more detailed information about the study.

Thank you for your time!

Sincerely,

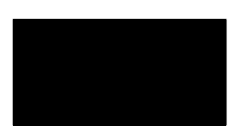

Dr. Mark Tremblay, PhD, Dlitt, FACSM, CSEP-CEP

Director, Healthy Active Living and Obesity Research

Children's Hospital of Eastern Ontario

Cover letter_v2_02 Oct 2018 


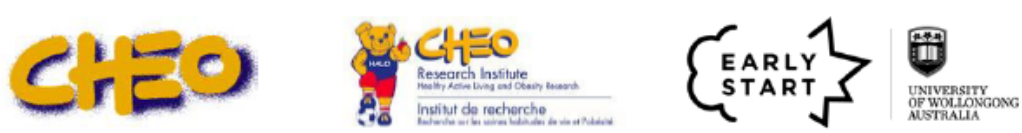

INFORMATION LETTER \& CONSENT FORM

PROTOCOL TITLE: International Surveillance Study of Movement Behaviours in the Early Years'

\section{MAIN INVESTIGATOR:}

Dr. Mark Tremblay

Healthy Active Living and Obesity (HALO) Research Group,

Children's Hospital of Eastern Ontario-Research Institute (CHEO-RI)

401 Smyth Rd. Room211, Ottawa, ON, K1H 8L1

Tel: 613-737-7600 x4114, Email: mtremblay@cheo.on.ca

Dear. Parents/Guardians,

You and your child are being invited to join in a research study entitled "International Surveillance Study of Movement Behaviours in the Early Years". You are being invited to join this study because your child is attending an elementary school/daycare centre that agreed to participate in this study. Before agreeing to take part in this study, it is important that you read and understand this document.

Taking part in this study is voluntary. Your decision to participate or not in this study will not affect the care you receive at CHEO or your child's school/daycare centre. You are free to withdraw from the study at any time and there will be no penalty to you or your child.

Why is this study being done?

Consider a young child's physical and motor development in the context of how they move throughout a typical day, It is usually a combination of sleeping, sitting, standing, and different intensities of physical activity, the latter mostly in the form of play and other activities of daily living. We know little about how these behaviours - individually and in combination - influence one another and how they relate to healthy growth and development. The primary aim of this study is to determine the proportion of 4-year-old children who meet the World Health Organization (WHO) Global 24hour Movement Guidelines for the Early Years.

How many people will participate?

A total of 100 children who attend a local elementary school/daycare centre and their parents from either urban or rural areas in Canada (Ottawa, ON Region) will be asked to participate in this study. The same sampling will be done in Brazil, China, Papua New Guinea and South Africa.

What will I have to do?

1. Activities, Games and Measurements: If you agree to allow your child to participate in the study, your child will be asked to do various activities with a data collector (throwing and catching a ball, balancing, solving puzzles, cutting paper, and drawing) as well as three games on an iPad. The iPad games ('Card Sort' task, 'Fish and Shark' task, and 'Mr. Ant' task) allow us to assess some of their cognitive abilities such as memory. The data collectors will also measure your child's height and weight. All of these activities will take approximately 30 minutes total to complete.

2. Parent Questionnaire: While your child is participating in this study, we will ask you to fill out the parent questionnaire which will take about 15 minutes to complete.

3. A Waist Activity Monitor: Your child will be asked to wear a lightweight activity monitor. The monitor will be worn around their waist (secured on a belt) for 72 hours to measure their movement behaviours (e.g., physical activity,

Information letter and consent form v10_20190304 
sitting, sleep; Figure 1). It is important to wear this belt at all times throughout the day and night except during water-based activities, since it is not waterproof. The child or parents/guardians will not be held responsible for any loss or damage to these devices. There is a separate sheet with further information about the monitor titled 'Activity Monitors Instruction Sheet'.

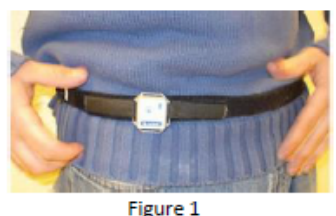

Are there any risks to participatina?

Apart from the time taken to participate in this study, there are no known risks associated with taking part in this study. A potential discomfort may include you feeling uncomfortable with some of the questions being asked if they are sensitive. If you feel uncomfortable, you may choose not to answer a question. Also, your child may feel uncomfortable wearing the activity monitor for 72 hours. If this is the case, your child may choose to take off the monitor.

Are there any benefits to participating?

If you decide to participate, you may or may not benefit from participating in this study. However, we hope to identify areas that we can focus on to promote healthy growth, development and well-being of young children from around the world.

Will I be paid to participate?

You will not be paid to take part in this study.

Can I withdraw?

You and your child's involvement in the study is entirely voluntary and you may decline to participate, or withdraw from the study, at any time. You can withdraw from the study by contacting Dr. Mark Tremblay (mtremblay@cheo.on.ca). Your child's data can also be withdrawn, by requesting this in writing within two weeks of the program's completion. In either event, declining to participate or withdrawing from the study will not affect your relationship with the researchers, your child's elementary school/daycare centre or the CHEO.

What about confidentiality and privacy?

Your personal information will be kept strictly confidential except as required or permitted by law.

For this study we will be collecting personal identifiers from you (i.e., name, age, sex, highest level of education, postal code, phone number and email address) and your child (i.e., name, date of birth, sex, and race/ethnicity) for the research purposes described in this letter. Your personal identifiers will be kept in a document that links this information with a study ID, called a master list. The study ID will be used in all of the research documents instead of your personal information to protect your privacy. The master list will be stored separately from the research data. It will be stored in a password locked computer in a CHEO-RI lab with access restricted to the principal investigator and the study team. Representatives from the CHEO Research Ethics Board (REB) and a quality reviewer from CHEO-RI may look at your records at the site where these records are held, to check that the study is following the proper laws and guidelines.

The data produced from this study will be stored in a password secured computer. Only members of the study team and the individuals described above will have access to the data. Following completion of the study the data and master list will be kept for 7 years after the last publication of this study. They will then be destroyed. You will not be identified in any publication or presentation of this study.

This study is taking place at multiple sites around the world which means the research data will be shared outside of

Information letter and consent form v10_20190304 
the CHEO-RI. The research coordinating centre, the Early Start Research Institute (ESRI) at the University of Wollongongin Australia, will receive data including your study ID, for data analysis and/or quality assurance. Any personal information about you that leaves the CHEO-RI will be coded with a study ID so that you cannot be identified by name. This is called de-identified data. The de-identified data will be sent securely to the ESRI and stored in a password secured computer for 5 years.

Is the research team benefiting from the study?

The research team members are not benefiting personally, financially or in some other way from this study. The benefit of the study is increasing our understanding of healthy movement behaviours of young children.

Will I be told about new information?

Please let the study team or Dr. Mark Tremblay (mtremblay@cheo.on.ca) know if you would like to receive a copy of the research findings.

What if I have questions?

This study has been reviewed and approved by The Ottawa-Carleton Research and Evaluation Advisory Committee and the CHEO REB. The CHEO REB is a committee of the hospital that includes individuals from different professional backgrounds. The Board reviews all human research that takes place at the hospital. Its goal is to ensure the safety of people taking part in research. The Board's work is not meant to replace a parent or child's decisions or choices that are best for them. You may contact the Research Ethics Board for information regarding a patient's rights in research studies at (613) 737-7600 (3272), although this person cannot provide any health-related information about the study.

If you have any questions concerning participation in this study, please do not hesitate to contact the Principal Investigator Dr. Mark Tremblay (mtremblay@cheo.on.ca).

Next steps to participation

If you feel that you have enough information about the study and do not have any further questions, feel free to review this information sheet, sign and return the consent form to your child's teacher or daycare provider. Please also complete and return the attached questionnaire providing basic demographic information on factors, such as age, sleep time, screen time, and others. If you do have any further questions, please feel free to contact Dr. Mark Tremblay (mtremblay@cheo.on.ca). 


\title{
CONSENT FORM
}

\author{
I (your full name) \\ agree to take part in the pilot study \\ entitled: "International Surveillance Study of Movement Behaviours in the Early Years" and for my child to \\ participate in the following assessments (approximately 30 minutes to complete): \\ - Have their cognitive ability measured by playing 3 different games using an iPad; \\ - Have their motor skills assessed by completing various activities (throwing, catching etc.); \\ - Have their body measurements taken (height \& weight); and \\ - Have their physical activity measured by an activity monitors for 3 days.
}

By signing this consent form, I agree that:

- I am voluntarily agreeing for my child and I to participate in this study;

- I understand the information in the information letter;

- All of the risks and benefits of participation have been explained to me;

- All of my questions have been answered;

- I allow access to my personal information as described in this consent form;

- I do not give up my legal rights by signing this form;

- I understand that me or my child will not be held responsible for any loss or damage to the activity monitors; and

- I understand that a copy of the signed consent form will be provided to me.

Child's Full Name:

Parent/Guardian's signature:

Date: 


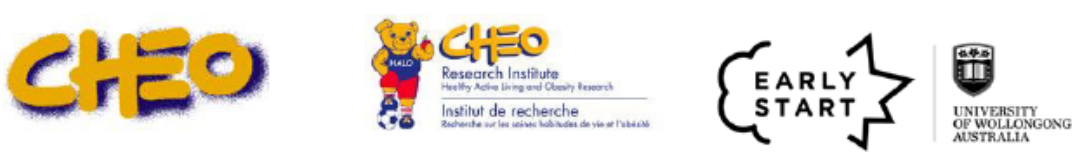

\section{CONTACT INFORMATION SHEET}

Name of Elementary School /Childcare Centre:

Child's full name:

First Last

Name of Parent/ Guardian:

First

Last

Parent/ Guardian Phone Number:

)

Parent/ Guardian Alternate Phone Number: $\_($

Parent/ Guardian Email Address:

Postal Code:

Please check how you prefer to be contacted:

Email $\square$

Phone $\square$

No contact $\square$ 


\section{APPENDIX 3. Parent/Guardian Questionnaire}

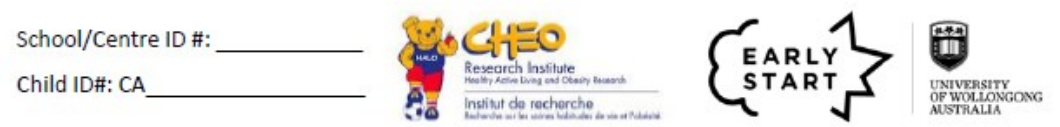

\section{PARENT/GUARDIAN QUESTIONNAIRE}

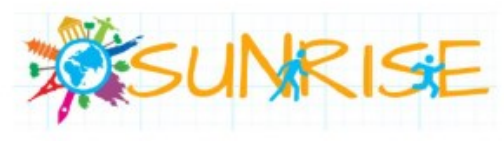

\section{INTERNATIONAL SURVEILLANCE STUDY OF 24-HOUR MOVEMENT BEHAVIOURS IN THE EARLY YEARS: The SUNRISE Study}

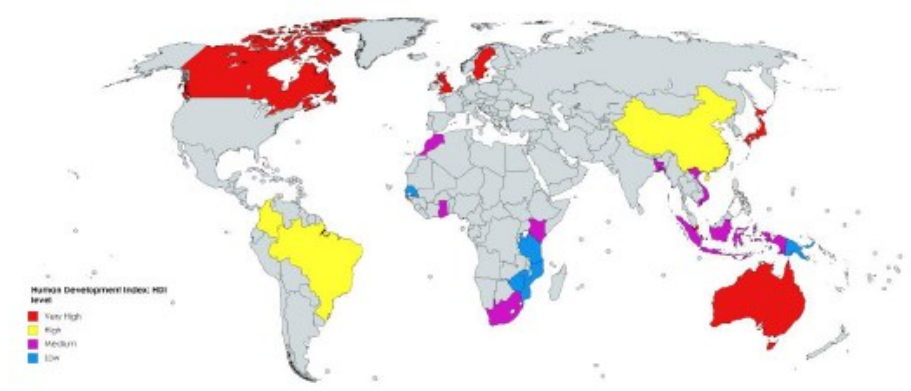

Instructions: This questionnaire is for parents/guardians who have a child approximately aged 4 years old (48-59 months old). Please take your time and read each question carefully. Choose the answer that best describes you and your child by placing a checkmark ( $v$ ) in the box provided or writing in the space provided. If there is a question that you do not want to answer, you do not have to. Your responses will be kept confidential.

Parent Questionnaire V5 02/Oct/2018 


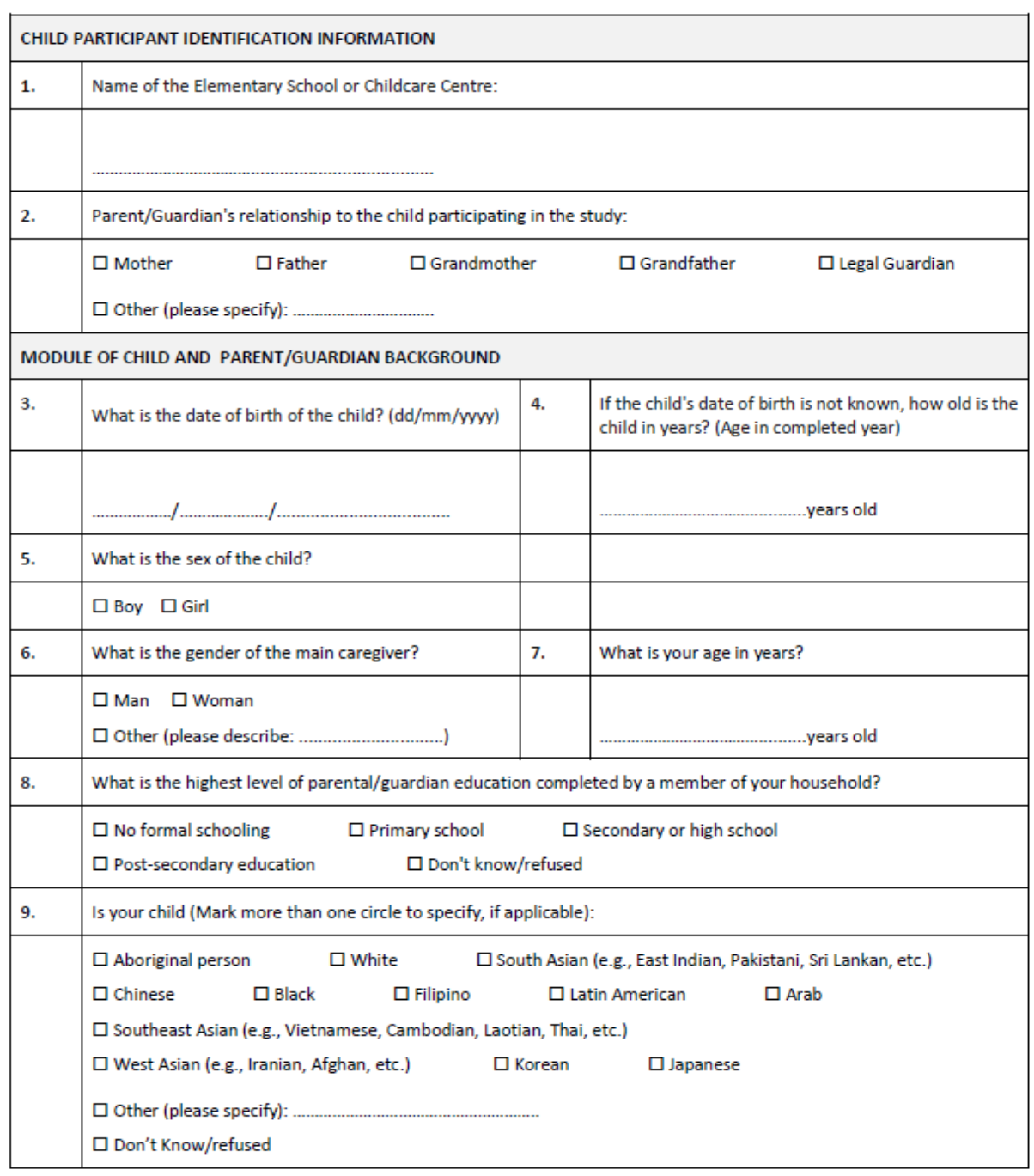

QUALITY CONTROL

DATA ENTRY
Staff initials:

Staff initials:
Date:

Date: 


\begin{tabular}{|c|c|c|c|c|}
\hline \multicolumn{5}{|c|}{ CORE: PHYSICAL ACTIVITY, SCREEN TIME, SEDENTARY BEHAVIOUR, AND SLEEP } \\
\hline \multicolumn{5}{|c|}{$\begin{array}{l}\text { The next questions ask about your child's movement behaviours. } \\
\text { Please report the number of hours and minutes per day (for all the questions). E.g. } 1 \text { hour and } 30 \text { mins }\end{array}$} \\
\hline 10. & \multicolumn{4}{|c|}{$\begin{array}{l}\text { In a } 24 \text { hour period in the past week, how much time did the 3-5 year old child who is participating in this study spend } \\
\text { on: }\end{array}$} \\
\hline 10a. & \multicolumn{3}{|c|}{$\begin{array}{l}\text { In a variety of physical activities, spread throughout the day? } \\
\text { Physical activity includes active movements, such as going for a } \\
\text { walk, skating, running around, playing at a spray park or pool, } \\
\text { riding a tricycle, gymnastics, dancing, playing at the park, } \\
\text { playground, or in the yard. }\end{array}$} & 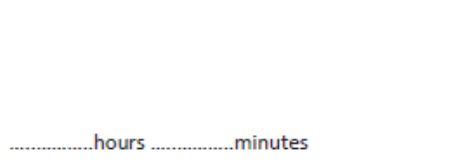 \\
\hline $10 \mathrm{~b}$. & \multicolumn{3}{|c|}{$\begin{array}{l}\text { In energetic play that causes him/her to 'huff and puff' and } \\
\text { increases his/her heart rate (this would be part of the total time } \\
\text { spent in a variety of activities just mentioned). }\end{array}$} & 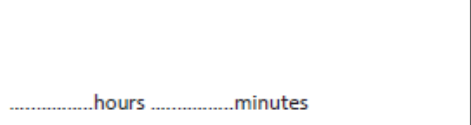 \\
\hline 10c. & \multicolumn{3}{|c|}{$\begin{array}{l}\text { Using anv electronic screen device such as a smart phone, tablet, } \\
\text { video game, or watch television or movies, videos on the internet } \\
\text { while they were sitting or lying down? }\end{array}$} & 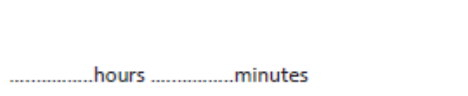 \\
\hline 11. & \multicolumn{4}{|c|}{ In the past week, were there any days where your child who is participating in this study: } \\
\hline \multirow[t]{2}{*}{ 11a. } & $\begin{array}{l}\text { Was re } \\
\text { stroller }\end{array}$ & $\begin{array}{l}\text { e than one hour at a time in a } \\
\text { gh chair/booster seat? }\end{array}$ & $11 b$. & Sat for more than 30 minutes at a time? \\
\hline & $\square$ Yes & $\square$ No & & $\square$ Yes \\
\hline 12. & \multicolumn{3}{|c|}{$\begin{array}{l}\text { How many hours of sleep does this child get in a typical 24-hours } \\
\text { day (including naps)? }\end{array}$} & 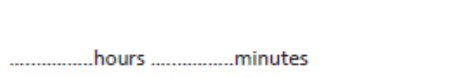 \\
\hline \multirow[t]{2}{*}{13.} & \multicolumn{4}{|c|}{$\begin{array}{l}\text { Does the child use electronic screen devices (e.g. TV, video game, computer, tablet or smartphone) in the } 2 \text { hours } \\
\text { before bedtime on a daily basis? }\end{array}$} \\
\hline & $\square$ Yes & $\square$ No & \multicolumn{2}{|c|}{$\square$ Don't know } \\
\hline \multirow[t]{2}{*}{14.} & \multicolumn{4}{|c|}{ If Yes, how close to bedtime does the child usually use these devices? If no, go to question 23.} \\
\hline & \multicolumn{4}{|c|}{$\begin{array}{l}\square \text { Closer than } 30 \text { minutes before bedtime } \\
\square 30 \text { minutes to less than } 1 \text { hour before bedtime } \\
\square \text { Between } 1 \text { and } 2 \text { hours before bedtime }\end{array}$} \\
\hline \multirow[t]{2}{*}{15.} & \multicolumn{4}{|c|}{$\begin{array}{l}\text { Does the child have electronic screen devices in the room where he/she sleeps (e.g. TV, video game, computer, tablet } \\
\text { or smartphone)? }\end{array}$} \\
\hline & $\square$ Yes & $\square$ No & & \\
\hline
\end{tabular}

QUALITY CONTROL

DATA ENTRY
Staff initials:

Staff initials:
Date:

Date: 


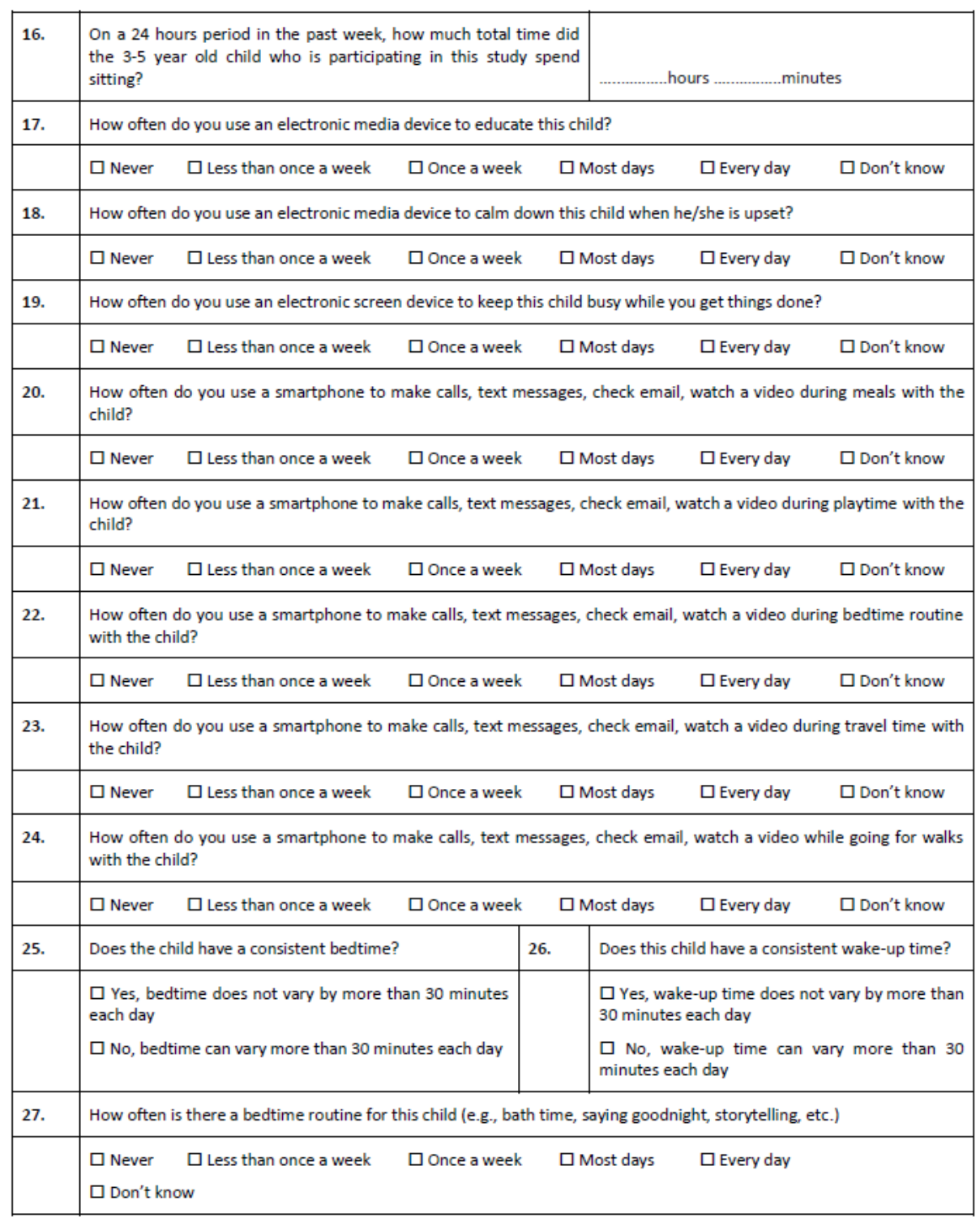

QUALITY CONTROL DATA ENTRY
Staff initials: Staff initials:
Date:

Date: 


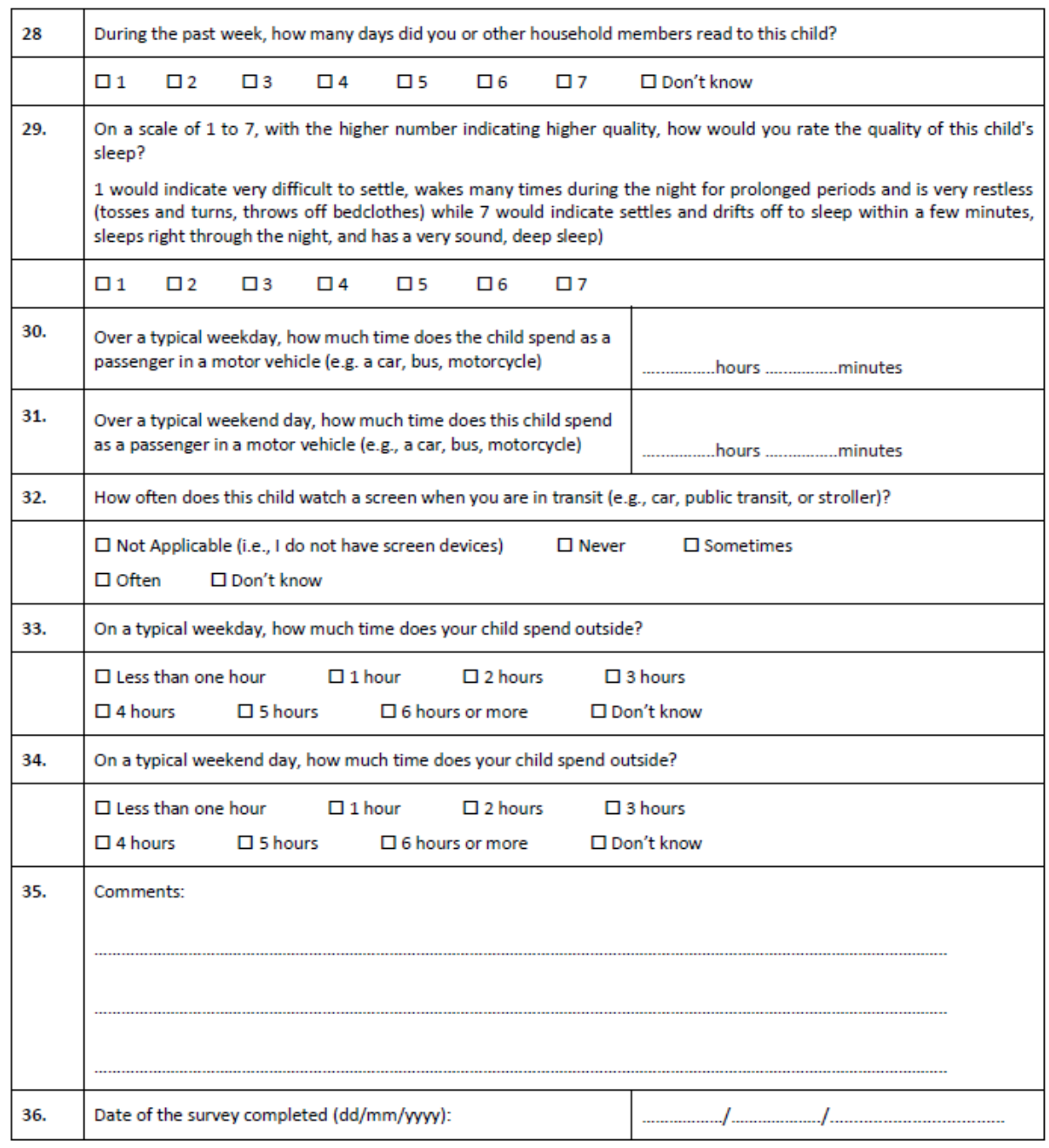

This is the end of the questionnaire. Thank you very much for your participation! Please return the questionnaire in the sealed envelope to the educator at your child's school or daycare centre.

QUALITY CONTROL DATA ENTRY
Staff initials:

Staff initials:

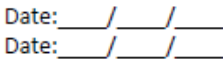


ID:

\section{Ages and Stages Questionnaire}

\section{GROSS MOTOR}

1. Does your child catch a large ball with both hands? (You should stand about 5 feet away and give your child two or three tries before you mark the answer.)

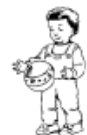

2. Does your child climb the rungs of a ladder of a playground slide and slide down without help?

3. While standing, does your child throw a ball overhand in the direction of a person standing at least 6 feet away? To throw overhand, your child must raise his arm to shoulder height and throw the ball forward. (Dropping the ball or throwing the ball underhand should be scored as "not yet.")

Does your child hop up and down on either the right or left foot at least one time without losing her balance or falling?

5. Does your child jump forward a distance of 20 inches from a standing position, starting with his feet together?

6. Without holding onto anything, does your child stand on one foot for at least 5 seconds without losing her balance and putting her foot down? (You may give your child two or three tries before you mark the answer.?

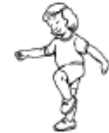

\section{FINE MOTOR}

1. Does your child put together a five- to seven-piece interlocking puzzle? (If one is not available, take a full-page picture from a magazine or catalog and cut it into six pieces. Does your child put it back together correctly?)

2. Using child-safe scissors, does your child cut a paper in half on a more or less straight line, making the blades go up and down? (Carefully watch your child's use of scissors for safety reasons.

3. Using the shapes below to look at, does your child copy at least three shapes onto a large piece of paper using a pencil, crayon, or pen, with out tracing? (Your child's drawings should look similar to the design of the shapes below, but they may be different in size.)

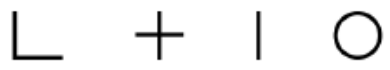

4. Does your child unbutton one or more buttons? (Your child may use his own clothing or a doll's clothing.)

5. Does your child draw pictures of people that have at least three of the following features: head, eyes, nose, mouth, neck, hair, trunk, arms, hands, legs, or feet?

6. Does your child color mostly within the lines in a coloring book or within the lines of a 2-inch circle that you draw? (Your child should not go more than $1 / 4$ inch outside the lines on most of the picture.)
Yes SOMETIMES NOT Yet<smiles>[C-]1CO1</smiles><smiles>[CH-]CO</smiles><smiles>C=CO</smiles>

○

$\bigcirc$

$\bigcirc-$

O

O

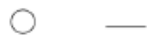

$\bigcirc$

0

$\bigcirc$

GROSS MOTOR TOTAL

YES

SOMETIMES NOT YET

0

0

O

0

O

0

Q

0

FINE MOTOR TOTAL 


\section{APPENDIX 5. Parent/Guardian Feedback Form

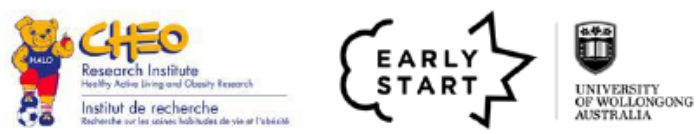

\section{Parent/Guardian Feedback}

1. How did you find the parent/guardian questionnaire? Were there any questions that you didn't understand or found hard to answer?

2. Did your child enjoy participating? Was there anything you think they did not enjoy or that could be improved?

3. How did you find it went for your child wearing the activity monitor for three days? Were there any problems? If so, can you tell us about them and offer any advice on how we could improve this?

4. Do you have any other comments to make?

This is the end of the questionnaire. Thank you very much for your participation! Please return the questionnaire in the sealed envelope to the educator at your child's school/centre. 


\section{APPENDIX 6. Staff Feedback Form}

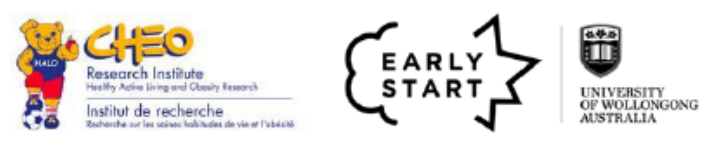

\section{Staff Feedback}

1. How did you find having the data collection team in your school/centre this week? Is there anything that you could recommend be done differently if the study was to be repeated to minimize any disruption to your daily routines?

2. How do you think the children found the gross and fine motor skills activities and the iPad games? Do you have any feedback or advice on how the assessment of these items could be improved?

3. Did the children enjoy participating? Was there anything you think they did not enjoy or that could be improved?

4. How did you find it went for the children wearing the activity monitor for three days? Were there any problems? If so, can you tell us about them and offer any advice on how we could improve this

5. Do you have any other comments to make?

This is the end of the questionnaire. Thank you very much for your participation!

Please return the questionnaire in the sealed envelope to the educator at your child's school/centre. 


\section{References}

1. Black MM, Walker SP, Fernald LCH, et al. Early Childhood Development Coming of Age: Science Through the Life Course. Lancet. 2017;389(10064):77-90.

2. WHO. WHO Guidelines on Physical Activity, Sedentary Behaviour and Sleep for Children under 5 Years of Age. Geneva: World Health Organization; 2019.

3. Tremblay MS, Chaput J-P, Adamo KB, et al. Canadian 24-Hour Movement Guidelines for the Early Years ( $0-4$ years): An Integration of Physical Activity, Sedentary Behaviour, and Sleep. BMC Public Health. 2017;17(S5):874.

4. Okely AD, Ghersi D, Hesketh KD, et al. A Collaborative Approach to Adopting/Adapting Guidelines - The Australian 24-Hour Movement Guidelines for the Early Years (Birth to 5 years): An Integration of Physical Activity, Sedentary Behavior, and Sleep. BMC Public Health. 2017;17(S5):869.

5. WHO. Human Development Report 2019. Geneva: World Health Organization; 2019.

6. Sameroff AJ. The Transactional Model of Development : How Children and Contexts Shape Each Other. Washington, DC: American Psychological Association; 2009.

7. Gluckman PD, Hanson MA, Low FM. The Role of Developmental Plasticity and Epigenetics in Human Health. Birth Defects Res Part C Embryo Today Rev. 2011;93(1):12-18. 
8. Jacob CM, Baird J, Barker M, et al. The Importance of a Life Course Approach to Health: Chronic Disease Risk from Preconception through Adolescence and Adulthood. Geneva: World Health Organization; 2017.

9. Oestreich AK, Moley KH. Developmental and Transmittable Origins of Obesity-Associated Health Disorders. Trends Genet. 2017;33(6):399-407.

10. Barker DJP. The Origins of the Developmental Origins Theory. J Intern Med. 2007;261(5):412-417.

11. Rose G. Familial Patterns in Iscaemic Heart Disease. Br J Prev Soc Med. 1964;18(2):75-80.

12. Buck C, Simpson H. Infant Diarrhoea and Subseqeunt Mortality from Heart Disease and Cancer. J Epidemiol Community Health. 1982;36(1):27-30.

13. Forsdahl A. Are Poor Living Conditions in Childhood and Adolescence an Important Risk Factor for Arteriosclerotic Heart Disease? Br J Prev Soc Med. 1977;31(2):91-95.

14. Barker DJP, Osmond C. Infant Mortality, Childhood Nutrition, and Ischaemic Heart Disease in England and Wales. Lancet. 1986;327(8489):1077-1081.

15. Heindel JJ, Vandenberg LN. Developmental Origins of Health and Disease: A Paradigm for Understanding Disease Etiology and Prevention. Curr Opin Pediatr. 2015;27(2):248-253.

16. Preston JD, Reynolds LJ, Pearson KJ. Developmental Origins of Health Span and Life Span: A Mini-Review. Gerontology. 2018;64(3):237-245.

17. Wibaek R, Vistisen D, Girma T, et al. Body Mass Index Trajectories in Early Childhood in Relation to Cardiometabolic Risk Profile and Body Composition 
at 5 Years of Age. Am J Clin Nutr. 2019;110(5):1175-1185.

18. Wibaek R, Girma T, Admassu B, et al. Higher Weight and Weight Gain after 4 Years of Age Rather than Weight at Birth Are Associated with Adiposity, Markers of Glucose Metabolism, and Blood Pressure in 5-Year-Old Ethiopian Children. J Nutr. 2019;149(10):1785-1796.

19. del Pozo-Cruz B, Perales F, Parker P, et al. Joint Physical-Activity/ScreenTime Trajectories During Early Childhood: Socio-Demographic Predictors and Consequences on Health-Related Quality-of-Life and Socio-Emotional Outcomes. Int J Behav Nutr Phys Act. 2019;16:55.

20. Wells JCK. The Capacity-Load Model of Non-Communicable Disease Risk: Understanding the Effects of Child Malnutrition, Ethnicity and the Social Determinants of Health. Eur J Clin Nutr. 2018;72(5):688-697.

21. Wells JCK. The Diabesity Epidemic in the Light of Evolution: Insights From the Capacity-Load Model. Diabetologia. 2019;62(10):1740-1750.

22. Hirvensalo M, Lintunen T. Life-Course Perspective for Physical Activity and Sports Participation. Eur Rev Aging Phys Act. 2011;8(1):13-22.

23. Royal College of Physicians and Surgeons of Canada. Early Childhood Development Royal College Position Statement. Ottawa, ON: Royal College of Physicians and Surgeons of Canada; 2014.

24. Kershaw P, Warburton B, Anderson L, et al. The Economic Costs of Early Vulnerability in Canada. Can J Public Health. 2010;101(S3):8-12.

25. Hertzman C. Social Geography of Developmental Health in the Early Years. Healthc Q. 2010;14(S1):32-40. 
26. Williams R, Clinton J, Canadian Paediatric Society, Early Years Task Force. Getting it Right at 18 Months: In Support of an Enhanced Well-Baby Visit. Paediatr Child Health. 2011;16(10):647-654.

27. Bouchard C, Blair SN, Haskell W. Physical Activity and Health. $2^{\text {nd }}$ ed. Windsor, ON: Human Kinetics; 2012.

28. WHO. WHO Global Action Plan on Physical Activity 2018-2030: More Active People for a Healthier World. Geneva: Wolds Health Organization; 2018.

29. Boreham C, Riddoch C. The Physical Activity, Fitness and Health of Children. J Sports Sci. 2001;19(12):915-929.

30. Norton K, Norton L, Sadgrove D. Position Statement on Physical Activity and Exercise Intensity Terminology. J Sci Med Sport. 2010;13(5):496-502.

31. Tremblay MS, Aubert S, Barnes JD, et al. Sedentary Behavior Research Network (SBRN)-Terminology Consensus Project Process and Outcome. Int $J$ Behav Nutr Phys Act. 2017;14(1):75.

32. Lee I-M, Shiroma EJ, Lobelo F, et al. Effect of Physical Inactivity on Major Non-Communicable Diseases Worldwide: An Analysis of Burden of Disease and Life Expectancy. Lancet. 2012;380(9838):219-229.

33. Kohl HW, Craig CL, Lambert EV, et al. The Pandemic of Physical Inactivity: Global Action for Public Health. Lancet. 2012;380(9838):294-305.

34. Tipton CM. Susruta of India, an Unrecognized Contributor to the History of Exercise Physiology. J Appl Physiol. 2008;104(6):1553-1556.

35. Warburton DER, Charlesworth S, Ivey A, et al. A Systematic Review of the Evidence for Canada's Physical Activity Guidelines for Adults. Int J Behav 
Nutr Phys Act. 2010;7:39.

36. Reiner M, Niermann C, Jekauc D, et al. Long-Term Health Benefits of Physical Activity - A Systematic Review of Longitudinal Studies. BMC Public Health. 2013;13(1):813.

37. Janssen I, LeBlanc AG. Systematic Review of the Health Benefits of Physical Activity and Fitness in School-Aged Children and Youth. Int J Behav Nutr Phys Act. 2010;7:40.

38. Poitras VJ, Gray CE, Borghese MM, et al. Systematic Review of the Relationships Between Objectively Measured Physical Activity and Health Indicators in School-Aged Children and Youth. Appl Physiol Nutr Metab. 2016;41(6 (Suppl. 3)):197-239.

39. Berk LE. Development Through the Lifespan. 7th ed. Hoboken, NJ: Pearson Education, Inc.; 2018.

40. Carson V, Lee E-Y, Hewitt L, et al. Systematic Review of the Relationships Between Physical Activity and Health Indicators in the Early Years (04 Years). BMC Public Health. 2017;17(S5):854.

41. Carson V, Hunter S, Kuzik N, et al. Systematic Review of Physical Activity and Cognitive Development in Early Childhood. J Sci Med Sport. 2016;19(7):573-578.

42. Figueroa R, An R. Motor Skill Competence and Physical Activity in Preschoolers: A Review. Matern Child Health J. 2017;21(1):136-146.

43. Hinkley T, Teychenne M, Downing KL, et al. Early Childhood Physical Activity, Sedentary Behaviors and Psychosocial Well-Being: A Systematic 
Review. Prev Med. 2014;62:182-192.

44. Timmons BW, LeBlanc AG, Carson V, et al. Systematic Review of Physical Activity and Health in the Early Years (Aged 0-4 Years). Appl Physiol Nutr Metab. 2012;37(4):773-792.

45. Ortega FB, Ruiz JR, Castillo MJ, et al. Physical Fitness in Childhood and Adolescence: A Powerful Marker of Health. Int J Obes. 2008;32(1):1-11.

46. Colley RC, Garriguet D, Adamo KB, et al. Physical Activity and Sedentary Behavior during the Early Years in Canada: A Cross-Sectional Study. Int $J$ Behav Nutr Phys Act. 2013;10:54.

47. Hesketh KR, McMinn AM, Ekelund U, et al. Objectively Measured Physical Activity in Four-Year-Old British Children: A Cross-Sectional Analysis of Activity Patterns Segmented Across the Day. Int J Behav Nutr Phys Act. 2014;11:1.

48. Hnatiuk JA, Salmon J, Hinkley T, et al. A Review of Preschool Children's Physical Activity and Sedentary Time Using Objective Measures. Am J Prev Med. 2014;47(4):487-497.

49. Malina RM. Physical Activity and Fitness: Pathways from Childhood to Adulthood. Am J Hum Biol. 2001;13(2):162-172.

50. Bingham DD, Costa S, Hinkley T, et al. Physical Activity During the Early Years: A Systematic Review of Correlates and Determinants. Am J Prev Med. 2016;51(3):384-402.

51. Bauman AE, Reis RS, Sallis JF, et al. Correlates of Physical Activity: Why Are Some People Physically Active and Others Not? Lancet. 
2012;380(9838):258-271.

52. Tudor-Locke CE, Myers AM. Challenges and Opportunities for Measuring Physical Activity in Sedentary Adults. Sport Med. 2001;31(2):91-100.

53. Hagstromer M, Ainsworth BE, Oja P, et al. Comparison of a Subjective and an Objective Measure of Physical Activity in a Population Sample. J Phys Act Health. 2010;7(4):541-550.

54. Prince SA, Adamo KB, Hamel ME, et al. A Comparison of Direct Versus SelfReport Measures for Assessing Physical Activity in Adults: A Systematic Review. Int J Behav Nutr Phys Act. 2008;5:56.

55. Strath SJ, Kaminsky LA, Ainsworth BE, et al. Guide to the Assessment of Physical Activity: Clinical and Research Applications: A Scientific Statement From the American Heart Association. Circulation. 2013;128(20):2259-2279.

56. Freedson PS, Miller K. Objective Monitoring of Physical Activity Using Motion Sensors and Heart Rate. Res Q Exerc Sport. 2000;71(S2):21-29.

57. Yang CC, Hsu YL. A Review of Accelerometry-Based Wearable Motion Detectors for Physical Activity Monitoring. Sensors. 2010;10(8):7772-7788.

58. Cliff DP, Reilly JJ, Okely AD. Methodological Considerations in Using Accelerometers to Assess Habitual Physical Activity in Children Aged 0-5 Years. J Sci Med Sport. 2009;12(5):557-567.

59. Kelly LA, Reilly JJ, Jackson DM, et al. Tracking Physical Activity and Sedentary Behavior in Young Children. Pediatr Exerc Sci. 2007;19(1):51-60.

60. Pate RR, Pfeiffer KA, Trost SG, et al. Physical Activity Among Children Attending Preschools. Pediatrics. 2004;114(5):1258-1263. 
61. Toschke JA, von Kries R, Rosenfeld E, et al. Reliability of Physical Activity Measures From Accelerometry Among Preschoolers in Free-Living Conditions. Clin Nutr. 2007;26(4):416-420.

62. Rowlands AV. Accelerometer Assessment of Physical Activity in Children: An Update. Pediatr Exerc Sci. 2007;19(3):252-266.

63. Ward DS, Evenson KR, Vaughn A, et al. Accelerometer Use in Physical Activity: Best Practices and Research Recommendations. Med Sci Sport Exerc. 2005;37(S11):582-588.

64. Corder K, Brage S, Ekelund U. Accelerometers and Pedometers: Methodology and Clinical Application. Curr Opin Clin Nutr Metab Care. 2007;10(5):597603.

65. Tremblay MS, Shephard RJ, Brawley LR. Research that Informs Canada's Physical Activity Guides: An Introduction. Can J Public Health. 2007;98(Suppl. 2):S1-S8; Appl Physiol Nutr Metab. 2007;32(Suppl. 2E):S1S8.

66. Tremblay MS, Warburton DER, Janssen I, et al. New Canadian Physical Activity Guidelines. Appl Physiol Nutr Metab. 2011;36(1):36-46.

67. Tremblay MS, LeBlanc AG, Carson V, et al. Canadian Physical Activity Guidelines for the Early Years (Aged 0-4 Years). Appl Physiol Nutr Metab. $2012 ; 37(2): 345-356$.

68. Katzmarzyk PT, Mason C. The Physical Activity Transition. J Phys Act Heal. 2009;6(3):269-280.

69. Guthold R, Stevens GA, Riley LM, et al. Global Trends in Insufficient 
Physical Activity Among Adolescents: A Pooled Analysis of 298 PopulationBased Surveys With 1.6 Million Participants. Lancet Child Adolesc Heal. 2020;4(1):23-35.

70. Sallis JF, Bull F, Guthold R, et al. Progress in Physical Activity Over the Olympic Quadrennium. Lancet. 2016;388(10051):1325-1336.

71. Janssen I. Health Care Costs of Physical Inactivity in Canadian Adults. Appl Physiol Nutr Metab. 2012;37(4):803-806.

72. Ding D, Lawson KD, Kolbe-Alexander TL, et al. The Economic Burden of Physical Inactivity: A Global Analysis of Major Non-Communicable Diseases. Lancet. 2016;388(10051):1311-1324.

73. Jetté M, Sidney K, Blümchen G. Metabolic Equivalents (METS) in Exercise Testing, Exercise Prescription, and Evaluation of Functional Capacity. Clin Cardiol. 1990;13(8):555-565.

74. Rhodes RE, Mark RS, Temmel CP. Adult Sedentary Behavior: A Systematic Review. Am J Prev Med. 2012;42(3):3-28.

75. Owen N, Healy GN, Matthews CE, et al. Too Much Sitting: The Population Health Science of Sedentary Behavior. Exerc Sport Sci Rev. 2010;38(3):105113.

76. Mansoubi M, Pearson N, Biddle SJH, et al. The Relationship Between Sedentary Behaviour and Physical Activity in Adults: A Systematic Review. Prev Med. 2014;69:28-35.

77. Proper KI, Singh AS, Van Mechelen W, et al. Sedentary Behaviors and Health Outcomes Among Adults: A Systematic Review of Prospective Studies. Am J 
Prev Med. 2011;40(2):174-182.

78. Wilmot EG, Edwardson CL, Achana FA, et al. Sedentary Time in Adults and the Association With Diabetes, Cardiovascular Disease and Death: Systematic Review and Meta-Analysis. Diabetologia. 2012;55(11):2895-2905.

79. Thorp AA, Owen N, Neuhaus M, et al. Sedentary Behaviors and Subsequent Health Outcomes in Adults: A Systematic Review of Longitudinal Studies, 1996-2011. Am J Prev Med. 2011;41(2):207-215.

80. Rezende LFM de, Rodrigues Lopes M, Rey-López JP, et al. Sedentary Behavior and Health Outcomes: An Overview of Systematic Reviews. PLoS One. 2014;9(8):e105620.

81. Carson V, Hunter S, Kuzik N, et al. Systematic Review of Sedentary Behaviour and Health Indicators in School-Aged Children and Youth: An Update. Appl Physiol Nutr Metab. 2016;41(6 (Suppl. 3)):240-265.

82. Cliff DP, Hesketh KD, Vella SA, et al. Objectively Measured Sedentary Behaviour and Health and Development in Children and Adolescents: Systematic Review and Meta-Analysis. Obes Rev. 2016;17(4):330-344.

83. Poitras VJ, Gray CE, Janssen X, et al. Systematic Review of the Relationships Between Sedentary Behaviour and Health Indicators in the Early Years (04 Years). BMC Public Health. 2017;17(S5):868.

84. Biddle SJH, Pearson N, Ross GM, et al. Tracking of Sedentary Behaviours of Young People: A Systematic Review. Prev Med. 2010;51(5):345-351.

85. Jones RA, Hinkley T, Okely AD, et al. Tracking Physical Activity and Sedentary Behavior in Childhood: A Systematic Review. Am J Prev Med. 
2013;44(6):651-658.

86. Temmel CSD, Rhodes R. Correlates of Sedentary Behaviour in Children and Adolescents Aged 7-18: A Systematic Review. Heal Fit J Canada. 2013;6(1):119-199.

87. Hinkley T, Salmon J, Okely AD, et al. Correlates of Sedentary Behaviours in Preschool Children: A Review. Int J Behav Nutr Phys Act. 2010;7:66.

88. Dias KI, White J, Jago R, et al. International Comparison of the Levels and Potential Correlates of Objectively Measured Sedentary Time and Physical Activity Among Three-to-Four-Year-Old Children. Int J Environ Res Public Health. 2019;16(11):E1929.

89. Yang L, Cao C, Kantor ED, et al. Trends in Sedentary Behavior Among the US Population, 2001-2016. JAMA. 2019;321(16):1587-1597.

90. Guthold R, Cowan MJ, Autenrieth CS, et al. Physical Activity and Sedentary Behavior Among Schoolchildren: A 34-Country Comparison. J Pediatr. 2010;157(1):43-49.

91. Arundell L, Fletcher E, Salmon J, et al. A Systematic Review of the Prevalence of Sedentary Behavior During the After-School Period Among Children Aged 5-18 Years. Int J Behav Nutr Phys Act. 2016;13:93.

92. Pereira JR, Cliff DP, Sousa-Sá E, et al. Prevalence of Objectively Measured Sedentary Behavior in Early Years: Systematic Review and Meta-Analysis. Scand J Med Sci Sports. 2019;29(3):308-328.

93. Tremblay MS, LeBlanc AG, Janssen I, et al. Canadian Sedentary Behaviour Guidelines for Children and Youth. Appl Physiol Nutr Metab. 2011;36:59-64. 
94. Tremblay MS, LeBlanc AG, Carson V, et al. Canadian Sedentary Behaviour Guidelines for the Early Years (Aged 0-4 Years). Appl Physiol Nutr Metab. 2012;37:370-380.

95. LeBlanc A, Gunnell K, Prince S, et al. The Ubiquity of the Screen: An Overview of the Risks and Benefits of Screen Time in Our Modern World. Transl J Am Coll Sport Med. 2017;2(17):104-113.

96. Hamer M, Stamatakis E, Mishra GD. Television- and Screen-Based Activity and Mental Well-Being in Adults. Am J Prev Med. 2010;38(4):375-380.

97. Hancox RJ, Milne BJ, Poulton R. Association Between Child and Adolescent Television Viewing and Adult Health: A Longitudinal Birth Cohort Study. Lancet. 2004;364(9430):257-262.

98. Carson V, Lee EY, Hesketh KD, et al. Physical Activity and Sedentary Behavior Across Three Time-Points and Associations With Social Skills in Early Childhood. BMC Public Health. 2019;19(1):27.

99. Ashton JJ, Beattie RM. Screen Time in Children and Adolescents: Is There Evidence to Guide Parents and Policy? Lancet Child Adolesc Heal. 2019;3(5):292-294.

100. Royal College of Paediatrics and Child Health. The Health Impacts of Screen Time: A Guide for Clinicians and Parents. London, UK: Royal College of Paediatrics and Child Health; 2019.

101. Salmon J, Tremblay MS, Marshall SJ, et al. Health Risks, Correlates, and Interventions to Reduce Sedentary Behavior in Young People. Am J Prev Med. 2011;41(2):197-206. 
102. Duch H, Fisher EM, Ensari I, et al. Screen Time Use in Children Under 3 Years Old: A Systematic Review of Correlates. Int J Behav Nutr Phys Act. $2013 ; 10: 102$.

103. LeBlanc AG, Katzmarzyk PT, Barreira TV, et al. Correlates of Total Sedentary Time and Screen Time in 9-11 Year-Old Children Around the World: The International Study of Childhood Obesity, Lifestyle and the Environment. PLoS One. 2015;10(6):e0129622.

104. Madigan S, Racine N, Tough S. Prevalence of Preschoolers Meeting vs Exceeding Screen Time Guidelines. JAMA Pediatr. 2020;174(1):93-95.

105. Garriguet D, Carson V, Colley R, et al. Physical Activity and Sedentary Behaviour of Canadian Children Aged 3 to 5. Health Rep. 2016;27(9):14-23.

106. Carson V, Tremblay MS, Spence JC, et al. The Canadian Sedentary Behaviour Guidelines for the Early Years (Zero to Four Years of Age) and Screen Time Among Children From Kingston, Ontario. Paediatr Child Health. $2013 ; 18(1): 25-28$.

107. Kuzik N, Poitras VJ, Tremblay MS, et al. Systematic Review of the Relationships Between Combinations of Movement Behaviours and Health Indicators in the Early Years (0-4 Years). BMC Public Health. 2017;17(S5):849.

108. Buysse DJ. Sleep Health: Can We Define It? Does It Matter? Sleep. 2014;37(1):9-17.

109. Czeisler CA. Impact of Sleepiness and Sleep Deficiency on Public Health Utility of Biomarkers. J Clin Sleep Med. 2011;7(S5):6-8. 
110. Crabtree VML, Williams NA. Normal Sleep in Children and Adolescents. Child Adolesc Psychiatr Clin N Am. 2009;18(4):799-811.

111. Chaput J-P, Gray CE, Poitras VJ, et al. Systematic Review of the Relationships Between Sleep Duration and Health Indicators in School-Aged Children and Youth. Appl Physiol Nutr Metab. 2016;41(6 (Suppl. 3)):266-282.

112. Matricciani L, Paquet C, Galland B, et al. Children's Sleep and Health: A Meta-Review. Sleep Med Rev. 2019;46:136-150.

113. Medic G, Wille M, Hemels MEH. Short- and Long-Term Health Consequences of Sleep Disruption. Nat Sci Sleep. 2017;9:151-161.

114. Cappuccio FP, D’Elia L, Strazzullo P, et al. Sleep Duration and All-Cause Mortality: A Systematic Review and Meta-Analysis of Prospective Studies. Sleep. 2010;33(5):585-592.

115. Yin J, Jin X, Shan Z, et al. Relationship of Sleep Duration With All-Cause Mortality and Cardiovascular Events: A Systematic Review and DoseResponse Meta-Analysis of Prospective Cohort Studies. J Am Heart Assoc. 2017;6(9):e005947.

116. Chaput JP, Gray CE, Poitras VJ, et al. Systematic Review of the Relationships Between Sleep Duration and Health Indicators in the Early Years (0-4 Years). BMC Public Health. 2017;17(S5):855.

117. Reynaud E, Vecchierini M-F, Heude B, et al. Sleep and its Relation to Cognition and Behaviour in Preschool-Aged Children of the General Population: A Systematic Review. J Sleep Res. 2018;27(3):e12636.

118. Belmon LS, van Stralen MM, Busch V, et al. What are the Determinants of 
Children's Sleep Behavior? A Systematic Review of Longitudinal Studies. Sleep Med Rev. 2019;43:60-70.

119. Peña M-M, Rifas-Shiman SL, Gillman MW, et al. Racial/Ethnic and SocioContextual Correlates of Chronic Sleep Curtailment in Childhood. Sleep. 2016;39(9):1653-1661.

120. Chang VC, Chaput J-P, Roberts KC, et al. Factors Associated with Sleep Duration Across Life Stages: Results From the Canadian Health Measures Survey. Heal Promot Chronic Dis Prev Can. 2018;38(11):404-418.

121. Ward TM, Gay C, Anders TF, et al. Sleep and Napping Patterns in 3-to-5-Year Old Children Attending Full-Day Childcare Centers. J Pediatr Psychol. 2008;33(6):666-672.

122. Acebo C, Sadeh A, Seifer R, et al. Sleep/Wake Patterns Derived From Activity Monitoring and Maternal Report for Healthy 1- to 5-Year-Old Children. Sleep. 2005;28(12):1568-1577.

123. Matricciani L, Olds T, Petkov J. In Search of Lost Sleep: Secular Trends in the Sleep Time of School-Aged Children and Adolescents. Sleep Med Rev. 2012;16(3):203-211.

124. Galland BC, Taylor BJ, Elder DE, et al. Normal Sleep Patterns in Infants and Children: A Systematic Review of Observational Studies. Sleep Med Rev. 2012;16(3):213-222.

125. Chaput J-P, Janssen I. Sleep Duration Estimates of Canadian Children and Adolescents. $J$ Sleep Res. 2016;25(5):541-548.

126. Michaud I, Chaput J-P. Are Canadian Children and Adolescents Sleep 
Deprived? Public Health. 2016;141:126-129.

127. Chaput J-P, Colley RC, Aubert S, et al. Proportion of Preschool-Aged Children Meeting the Canadian 24-Hour Movement Guidelines and Associations With Adiposity: Results From the Canadian Health Measures Survey. BMC Public Health. 2017;17(S5):829.

128. Hillman D, Mitchell S, Streatfeild J, et al. The Economic Cost of Inadequate Sleep. Sleep. 2018;41(8):zsy083.

129. Hafner M, Stepanek M, Taylor J, et al. Why Sleep Matters - The Economic Costs of Insufficient Sleep: A Cross-Country Comparative Analysis. Rand Health Q. 2017;6(4):11.

130. Hirshkowitz M, Whiton K, Albert SM, et al. National Sleep Foundation's Updated Sleep Duration Recommendations: Final Report. Sleep Heal. 2015;1(4):233-243.

131. Paruthi S, Brooks LJ, D'Ambrosio C, et al. Recommended Amount of Sleep for Pediatric Populations: A Consensus Statement of the American Academy of Sleep Medicine. J Clin Sleep Med. 2016;12(6):785-786.

132. Consensus Conference Panel, Watson NF, Badr MS, et al. Recommended Amount of Sleep for a Healthy Adult: A Joint Consensus Statement of the American Academy of Sleep Medicine and Sleep Research Society. Sleep. 2015;38(6):843-844.

133. Tremblay MS, Carson V, Chaput J-P, et al. Canadian 24-Hour Movement Guidelines for Children and Youth: An Integration of Physical Activity, Sedentary Behaviour, and Sleep. Appl Physiol Nutr Metab. 2016;41(6 (Suppl. 
3)):311-327.

134. Chaput JP. The Integration of Pediatric Sleep Health Into Public Health in Canada. Sleep Med. 2019;56:4-8.

135. Chaput JP, Carson V, Gray CE et al. Importance of All Movement Behaviors in a 24 Hour Period for Overall Health. Int J Environ Res Public Health. 2014;11(12):12575-12576.

136. Foti KE, Eaton DK, Lowry R, et al. Sufficient Sleep, Physical Activity, and Sedentary Behaviors. Am J Prev Med. 2011;41(6):596-602.

137. Laurson KR, Lee JA, Gentile DA, et al. Concurrent Associations between Physical Activity, Screen Time, and Sleep Duration with Childhood Obesity. ISRN Obes. 2014;2014:204540.

138. Chastin SFM, Palarea-Albaladejo J, Dontje ML, et al. Combined Effects of Time Spent in Physical Activity, Sedentary Behaviors and Sleep on Obesity and Cardio-Metabolic Health Markers: A Novel Compositional Data Analysis Approach. PLoS One. 2015;10(10):e0139984.

139. Carson V, Tremblay MS, Chaput J-P, et al. Associations Between Sleep Duration, Sedentary Time, Physical Activity, and Health Indicators Among Canadian Children and Youth Using Compositional Analyses. Appl Physiol Nutr Metab. 2016;41(6 (Suppl. 3)):294-302.

140. McGregor DE, Carson V, Palarea-Albaladejo J, et al. Compositional Analysis of the Associations Between 24-h Movement Behaviours and Health Indicators Among Adults and Older Adults From the Canadian Health Measure Survey. Int J Environ Res Public Health. 2018;15(8):E1779. 
141. Grgic J, Dumuid D, Bengoechea EG, et al. Health Outcomes Associated With Reallocations of Time Between Sleep, Sedentary Behaviour, and Physical Activity: A Systematic Scoping Review of Isotemporal Substitution Studies. Int J Behav Nutr Phys Act. 2018;15(1):69.

142. Saunders TJ, Gray CE, Poitras VJ, et al. Combinations of Physical Activity, Sedentary Behaviour and Sleep: Relationships With Health Indicators in School-Aged Children and Youth. Appl Physiol Nutr Metab. 2016;41(6 (Suppl. 3)):283-293.

143. Ministry of Health. Sit Less, Move More, Sleep Well Active Play Guidelines for Under-Fives. Wellington: Ministry of Health, New Zealand Government; 2017.

144. Draper CE, Tomaz SA, Biersteker L, et al. The South African 24-Hour Movement Guidelines for Birth to 5 Years: An Integration of Physical Activity, Sitting Behavior, Screen Time, and Sleep. J Phys Act Health. 2020;17(1):109-119.

145. Reilly JJ, Hughes AR, Janssen X, et al. GRADE-ADOLOPMENT Process to Develop 24-Hour Movement Behavior Recommendations and Physical Activity Guidelines for the Under 5s in the United Kingdom, 2019. J Phys Act Health. 2020;17(1):101-108.

146. U.S. Department of Health and Human Services. Physical Activity Guidelines for Americans, $2^{\text {nd }}$ edition. Washington, DC: U.S. Department of Health and Human Services; 2018.

147. Willumsen J, Bull F. Development of WHO Guidelines on Physical Activity, 
Sedentary Behavior, and Sleep for Children Less Than 5 Years of Age. J Phys Act Health. 2020;17(1):96-100.

148. Roman-Viñas B, Chaput JP, Katzmarzyk PT, et al. Proportion of Children Meeting Recommendations for 24-Hour Movement Guidelines and Associations With Adiposity in a 12-Country Study. Int J Behav Nutr Phys Act. 2016;13(1):123.

149. Janssen I, Roberts KC, Thompson W. Adherence to the 24-Hour Movement Guidelines Among 10- to 17-Year-Old Canadians. Heal Promot Chronic Dis Prev Canada. 2017;37(11):369-375.

150. Roberts KC, Yao X, Carson V, et al. Meeting the Canadian 24-hour movement guidelines for children and youth. Heal Reports. 2017;28(10):3-7.

151. Cliff DP, McNeill J, Vella SA, et al. Adherence to 24-Hour Movement Guidelines for the Early Years and Associations With Social-Cognitive Development Among Australian Preschool Children. BMC Public Health. 2017;17(S5):857.

152. Carson V, Ezeugwu VE, Tamana SK, et al. Associations Between Meeting the Canadian 24-Hour Movement Guidelines for the Early Years and Behavioral and Emotional Problems Among 3-Year-Olds. J Sci Med Sport. 2019;22(7):797-802.

153. Lee E-Y, Hesketh KD, Hunter S, et al. Meeting New Canadian 24-Hour Movement Guidelines for the Early Years and Associations With Adiposity Among Toddlers Living in Edmonton, Canada. BMC Public Health. 2017;17(S5):840. 
154. Manyanga T, Barnes JD, Chaput J-P, et al. Prevalence and Correlates of Adherence to Movement Guidelines Among Urban and Rural Children in Mozambique: A Cross-Sectional Study. Int J Behav Nutr Phys Act. 2019;16(1):94.

155. Lee EY, Carson V, Jeon JY, et al. Levels and Correlates of 24-Hour Movement Behaviors Among South Koreans: Results From the Korea National Health and Nutrition Examination Surveys, 2014 and 2015. J Sport Heal Sci. 2019;8(4):376-385.

156. Pearson N, Sherar LB, Hamer M. Prevalence and Correlates of Meeting Sleep, Screen-Time, and Physical Activity Guidelines among Adolescents in the United Kingdom. JAMA Pediatr. 2019;173(10):993-994.

157. Chen B, Bernard JY, Padmapriya N, et al. Socio-Demographic and Maternal Predictors of Adherence to 24-Hour Movement Guidelines in Singaporean Children. Int J Behav Nutr Phys Act. 2019;16(1):70.

158. Kracht CL, Webster EK, Staiano AE. Sociodemographic Differences in Young Children Meeting 24-Hour Movement Guidelines. J Phys Act Heal. 2019;16(10):908-915.

159. Machin D, Campbell MJ, Tan SB, et al. Sample Sizes for Clinical, Laboratory and Epidemiology Studies. Hoboken, NJ: John Wiley and Sons; 2018.

160. Morgan B, Hejdenberg J, Hinrichs-Krapels S, et al. Do Feasibility Studies Contribute to, or Avoid, Waste in Research. PLoS One. 2018;13(4):e0195951.

161. Orsmond GI, Cohn ES. The Distinctive Features of a Feasibility Study: Objectives and Guiding Questions. OTJR. 2015;35(3):169-177. 
162. Bowen DJ, Kreuter M, Spring B, et al. How We Design Feasibility Studies. Am J Prev Med. 2009;36(5):452-457.

163. Costa S, Barber SE, Cameron N, et al. The Objective Measurement of Physical Activity and Sedentary Behaviour in 2-3 Year Olds and Their ParentsL A Cross-Sectional Feasibility Study in the Bi-Ethnic Born in Bradford Cohort. BMC Public Health. 2015;15:1109

164. Müller J, Hoch AM, Zoller V, et al. Feasibility of Physical Activity Assessment with Wearable Devices in Children Aged 4-10 Years - A Pilot Study. Front Pediatr. 2018;6:5.

165. Tremblay MS, Barnes JD, González SA, et al. Global Matrix 2.0: Report Card Grades on the Physical Activity of Children and Youth Comparing 38 Countries. J Phys Act Heal. 2016;13(s2):343-366.

166. Aminian S, Hinckson EA. Examining the Validity of the activPAL Monitor in Measuring Posture and Ambulatory Movement in Children. Int J Behav Nutr Phys Act. 2012;9(1):119.

167. Pfeiffer KA, McIver KL, Dowda M, et al. Validation and Calibration of the Actical Accelerometer in Preschool Children. Med Sci Sports Exerc. $2006 ; 38(1): 152-157$.

168. GeoSearch. Statistics Canada. https://www12.statcan.gc.ca/censusrecensement/2016/geo/geosearch-georecherche/index-eng.cfm. Accessed February 11, 2020.

169. From Urban Areas to Population Centres. Statistics Canada. https://www.statcan.gc.ca/eng/subjects/standard/sgc/notice/sgc-06. Accessed 
May 27, 2019.

170. De Onis M, Wijnhoven TMA, Onyango AW. Worldwide Practices in Child Growth Monitoring. J Pediatr. 2004;144(4):461-465.

171. WHO, United Nations Children's Fund. WHO Child Growth Standards and the Identification of Severe Acute Malnutrition in Infants and Children. Geneva: World Health Organization; 2009.

172. Anderson LN, Carsley S, Lebovic G, et al. Misclassification of Child Body Mass Index From Cut-Points Defined by Rounded Percentiles Instead of ZScores. BMC Res Notes. 2017;10(1):639.

173. John D, Freedson P. ActiGraph and Actical Physical Activity Monitors: A Peek Under the Hood. Med Sci Sports Exerc. 2012;44(S1):86-89.

174. Johnson M, Meltz K, Hart K, et al. Validity of the Actical Activity Monitor for Assessing Steps and Energy Expenditure During Walking. J Sports Sci. 2015;33(8):769-776.

175. Adolph AL, Puyau MR, Vohra FA, et al. Validation of Uniaxial and Triaxial Accelerometers for the Assessment of Physical Activity in Preschool Children. J Phys Act Health. 2012;9(7):944-953.

176. De Decker E, De Craemer M, Santos-Lozano A, et al. Validity of the ActivPAL $^{\mathrm{TM}}$ and the ActiGraph Monitors in Preschoolers. Med Sci Sport Exerc. 2013;45(10):2002-2011.

177. Chastin SFM, Dontje ML, Skelton DA, et al. Systematic Comparative Validation of Self-Report Measures of Sedentary Time Against an Objective Measure of Postural Sitting (activPAL). Int J Behav Nutr Phys Act. 
2018;15(1):21.

178. Kozey-Keadle S, Libertine A, Lyden K, et al. Validation of Wearable Monitors for Assessing Sedentary Behavior. Med Sci Sports Exerc. 2011;43(8):15611567.

179. Huang WY, Lee E-Y. Comparability of activPAL-Based Estimates of Meeting Physical Activity Guidelines for Preschool Children. Int J Environ Res Public Health. 2019;16(24):5146.

180. Edwardson CL, Winkler EAH, Bodicoat DH, et al. Considerations When Using the activPAL Monitor in Field-Based Research With Adult Populations. J Sport Heal Sci. 2017;6(2):162-178.

181. Lee LFR, Dall PM. Concurrent Agreement Between ActiGraph $\mathbb{R}$ and activPALR in Measuring Moderate to Vigorous Intensity Physical Activity for Adults. Med Eng Phys. 2019;74:82-88.

182. Janssen X, Cliff DP, Reilly JJ, et al. Validation and Calibration of the $\operatorname{activPAL}^{\mathrm{TM}}$ for Estimating METs and Physical Activity in 4-6 Year Olds. $J$ Sci Med Sport. 2014;17(6):602-606.

183. Riley L, Guthold R, Cowan M, et al. The World Health Organization STEPwise Approach to Noncommunicable Disease Risk-Factor Surveillance: Methods, Challenges, and Opportunities. Am J Public Health. 2016;106(1):7478.

184. Limbos MM, Joyce DP. Comparison of the ASQ and PEDS in Screening for Developmental Delay in Children Presenting for Primary Care. J Dev Behav Pediatr. 2011;32(7):499-511. 
185. Steenis LJP, Verhoeven M, Hessen DJ, et al. Parental and Professional Assessment of Early Child Development: The ASQ-3 and the Bayley-III-NL. Early Hum Dev. 2015;91(3):217-225.

186. Rothstein A, Miskovic A, Nitsch K. Brief Review of Psychometric Properties and Clinical Utility of the Ages and Stages Questionnaires, Third Edition for Evaluating Pediatric Development. Arch Phys Med Rehabil. 2017;98(4):809810.

187. Kerstjens JM, Bos AF, ten Vergert EMJ, et al. Support for the Global Feasibility of the Ages and Stages Questionnaire as Developmental Screener. Early Hum Dev. 2009;85(7):443-447.

188. Howard SJ, Melhuish E. An Early Years Toolbox for Assessing Early Executive Function, Language, Self-Regulation, and Social Development: Validity, Reliability, and Preliminary Norms. J Psychoeduc Assess. 2017;35(3):255-275.

189. Hofmann W, Schmeichel BJ, Baddeley AD. Executive Functions and SelfRegulation. Trends Cogn Sci. 2012;16(3):174-180.

190. Blair C, Razza RP. Relating Effortful Control, Executive Function, and False Belief Understanding to Emerging Math and Literacy Ability in Kindergarten. Child Dev. 2007;78(2):647-663.

191. Riggs NR, Jahromi LB, Razza RP, et al. Executive Function and the Promotion of Social-Emotional Competence. J Appl Dev Psychol. 2006;27(4):300-309.

192. Colley RC, Harvey A, Grattan KP, et al. Impact of Accelerometer Epoch Length on Physical Activity and Sedentary Behaviour Outcomes for 
Preschool-Aged Children. Heal Reports. 2014;25(1):3-9.

193. Shein WH, Fitrianto, A. A Comparative Study of Outliers Identification Methods in Univariate Data Set. Adv Sci Lett. 2017;23(2):1422-1427.

194. Larouche R, Eryuzlu S, Livock H, et al. Test-Retest Reliability and Convergent Validity of Measures of Children's Travel Behaviours and Independent Mobility. J Transp Heal. 2017;6:105-118.

195. LeBlanc AG, Broyles ST, Chaput J-P, et al. Correlates of Objectively Measured Sedentary Time and Self-Reported Screen Time in Canadian Children. Int J Behav Nutr Phys Act. 2015;12(1):38.

196. Adamo KB, Wilson S, Harvey ALJ, et al. Does Intervening in Childcare Settings Impact Fundamental Movement Skill Development? Med Sci Sports Exerc. 2016;48(5):926-932.

197. Slegers C, Zion D, Glass D, et al. Why Do People Participate in Epidemiological Research? Bioethical Inq. 2015;12:227-237.

198. Teschke K, Marino S, Chu R, et al. Public Opinions About Participating in Health Research. Can J Public Health. 101(2):159-164.

199. Adamo KB, Papadakis S, Dojeiji L, et al. Using Path Analysis to Understand Parents' Perceptions of Their Children's Weight, Physical Activity and Eating Habits in the Champlain Region of Ontario. Paediatr Child Health. 2010;15(9):e33-e41.

200. Shi Y, Huang WY, Yu JJ, et al. Compliance and Practical Utility of Continuous Wearing of activPAL ${ }^{\mathrm{TM}}$ in Adolescents. Pediatr Exerc Sci. 2019;31(3):363-369. 
201. Penpraze V, Reilly JJ, MacLean CM, et al. Monitoring of Physical Activity in Young Children: How Much is Enough? Pediatr Exerc Sci. 2006;18(4):483491.

202. Abreo KA, Borkhoff CM, Anderson LN, et al. Objectively Measuring Physical Activity in Early Childhood Using Accelerometers: Are 4 Days Enough? Can J Diabetes. 2015;39:38-39.

203. Hislop J, Law J, Rush R, et al. An Investigation Into the Minimum Accelerometry Wear Time for Reliable Estimates of Habitual Physical Activity and Definition of a Standard Measurement Day in Pre-School Children. Physiol Meas. 2014;35(11):2213-2228.

204. Singh A, Yeh CJ, Boone Blanchard S. Ages and Stages Questionnaire: A Global Screening Scale. Bol Med Hosp Infant Mex. 2017;74(1):5-12.

205. Kvestad I, Taneja S, Kumar T, et al. The Assessment of Developmental Status Using the Ages and Stages Questionnaire-3 in Nutritional Research in North Indian Young Children. Nutr J. 2013;12(1):50.

206. Gollenberg AL, Lynch CD, Jackson LW, et al. Concurrent Validity of the Parent-Completed Ages and Stages Questionnaires, 2nd Ed. With the Bayley Scales of Infant Development II in a Low-Risk Sample. Child Care Health Dev. 2010;36(4):485-490.

207. Sarker H, Anderson LN, Borkhoff CM, et al. Validation of Parent-Reported Physical Activity and Sedentary Time by Accelerometry in Young Children. BMC Res Notes. 2015;8(1):735.

208. Tucker P, Gilliland J. The Effect of Season and Weather on Physical Activity: 
A Systematic Review. Public Health. 2007;121(12):909-922.

209. Carson V, Spence JC, Cutumisu N, et al. Seasonal Variation in Physical Activity Among Preschool Children in a Northern Canadian City. Res Q Exerc Sport. 2010;81(4):392-399.

210. Soini A, Tammelin T, Sääkslahti A, et al. Seasonal and Daily Variation in Physical Activity Among Three-Year-Old Finnish Preschool Children. Early Child Dev Care. 2014;184(4):589-601.

211. Government of Canada. Temperature and Precipitation Across Canada: Canada's Changing Climate Report. Ottawa, ON: Government of Canada; 2019.

212. Tandon PS, Zhou C, Lozano P, et al. Preschoolers' Total Daily Screen Time at Home and by Type of Child Care. J Pediatr. 2011;158(2):297-300.

213. Bernstein JPK, DeVito A, Calamia M. Subjectively and Objectively Measured Sleep Predict Differing Aspects of Cognitive Functioning in Adults. Arch Clin Neuropsychol. 2019;34(7):1127-1137.

214. Aubert S, Barnes JD, Abdeta C, et al. Global Matrix 3.0 Physical Activity Report Card Grades for Children and Youth: Results and Analysis From 49 Countries. J Phys Act Heal. 2018;15(S2):251-273.

215. Liu Y, Tang Y, Cao ZB, et al. Results From the China 2018 Report Card on Physical Activity for Children and Youth. J Exerc Sci Fit. 2019;17(1):3-7.

216. Xie G, Deng Q, Cao J, et al. Digital Screen Time and its Effect on Preschoolers' Behavior in China: Results From a Cross-Sectional Study. Ital J Pediatr. 2020;46(1):9. 
217. Bell L, Ullah S, Olds T, et al. Prevalence and Socio-Economic Distribution of Eating, Physical Activity and Sedentary Behaviour Among South Australian Children in Urban and Rural Communities: Baseline Findings From the OPAL Evaluation. Public Health. 2016;140:196-205.

218. Neto FA, Eto FN, Pereira TSS, et al. Active and Sedentary Behaviours in Children Aged 7 to 10 Years Old: The Urban and Rural Contexts, Brazil. BMC Public Health. 2014;14(1):1174.

219. Raymond SU, Leeder S, Greenberg HM. Obesity and Cardiovascular Disease in Developing Countries: A Growing Problem and an Economic Threat. Curr Opin Clin Nutr Metab Care. 2006;9(2):111-116.

220. Aminian S. Using the activPAL Monitor to Quantify Time Spent Sitting, Standing and Stepping at School: A One-day Snapshot. J Sci Res Reports. 2014;3(6):866-873.

221. Oliver M, Badland HM, Shepherd J, et al. Counting Steps in Research: A Comparison of Accelerometry and Pedometry. Open J Prev Med. 2011;01(01):1-7.

222. Van Cauwenberghe E, Wooller L, Mackay L, et al. Comparison of Actical and activPAL Measures of Sedentary Behaviour in Preschool Children. J Sci Med Sport. 2012;15(6):526-531.

223. Xu M, Fralick D, Zheng JZ, et al. The Differences and Similarities Between Two-Sample T-Test and Paired T-Test. Shanghai Arch Psychiatry. 2017;29(3):184-188.

224. Ranganathan P, Pramesh C, Aggarwal R. Common Pitfalls in Statistical 
Analysis: Measures of Agreement. Perspect Clin Res. 2017;8(4):187-191. 